\title{
A General-Purpose Contact Detection Algorithm for Nonlinear Structural Analysis Codes*
}

\author{
M.W. Heinstein \\ Engineering Mechanics and Material Modeling Department \\ S.W. Attaway \\ Computational Mechanics and Visualization Department \\ J.W. Swegle \\ Experimental Impact Physics Department \\ F.J. Mello \\ Solid and Structural Mechanics Department \\ Sandia National Laboratories \\ Albuquerque, New Mexico 87185
}

\begin{abstract}
A new contact detection algorithm has been developed to address difficulties associated with the numerical simulation of contact in nonlinear finite element structural analysis codes. Problems including accurate and efficient detection of contact for self-contacting surfaces, tearing and eroding surfaces, and multi-body impact are addressed. The proposed algorithm is portable between dynamic and quasi-static codes and can efficiently model contact between a variety of finite element types including shells, bricks, beams and particles. The algorithm is composed of (1) a location strategy that uses a global search to decide which slave nodes are in proximity to a master surface and (2) an accurate detailed contact check that uses the projected motions of both master surface and slave node. In this report, currently used contact detection algorithms and their associated difficulties are discussed. Then the proposed algorithm and how it addresses these problems is described. Finally, the capability of the new algorithm is illustrated with several example problems.
\end{abstract}

* This work performed at Sandia National Laboratories supported by the U.S. Department of Energy under contract DE-AC04-76DP00789 


\section{Nomenclature}

\begin{tabular}{|c|c|}
\hline$\Delta \mathrm{t}_{\mathrm{c}}$ & incremental time to contact \\
\hline$\xi, \eta$ & local surface coordinates of contact poirt \\
\hline a & i component of surface normal \\
\hline$B^{i}$ & bucket containing node $\mathrm{i}$ \\
\hline b & j component of surface normal \\
\hline$b_{s}$ & bucket size \\
\hline $\mathrm{C}_{3 \mathrm{D}}$ & $\begin{array}{l}\text { number of slave node-master nodes comparisons made in 3D bucket } \\
\text { sorting }\end{array}$ \\
\hline c & $\vec{k}$ component of surface normal \\
\hline $\overrightarrow{\mathrm{d}}_{\mathrm{ms}}$ & vector from master surface node to slave node \\
\hline $\mathrm{dt}$ & current time step \\
\hline$I_{x}, I_{y}, I_{z}$ & Index-vector for the $\mathrm{x}, \mathrm{y}$, and $\mathrm{z}$ coordinate respectively \\
\hline ibox $_{\min }$ & $\begin{array}{l}\text { slice of data containing minimum } x \text {-coordinate of master surface } \\
\text { capture box }\end{array}$ \\
\hline ibox $_{\max }$ & $\begin{array}{l}\text { slice of data containing maximum x-coordinate of master surface } \\
\text { capture box }\end{array}$ \\
\hline jbox $_{\min }$ & $\begin{array}{l}\text { slice of data containing minimum y-coordinate of master surface } \\
\text { capture box }\end{array}$ \\
\hline jbox $_{\max }$ & $\begin{array}{l}\text { slice of data containing maximum } y \text {-coordinate of master surface } \\
\text { capture box }\end{array}$ \\
\hline kbox $_{\min }$ & $\begin{array}{l}\text { slice of data containing minimum } z \text {-coordinate of master surface } \\
\text { capture box }\end{array}$ \\
\hline kbox $_{\max }$ & $\begin{array}{l}\text { slice of data containing maximum } \mathrm{z} \text {-coordinate of master surface } \\
\text { capture box }\end{array}$ \\
\hline$\left(i_{x}\right)_{\min }$ & $\begin{array}{l}\text { pointer into the Index vector corresponding to the first slave node } \\
\text { inside a master surface capture box along the x-coordinate }\end{array}$ \\
\hline$\left(i_{x}\right)_{\max }$ & $\begin{array}{l}\text { pointer into the Index vector corresponding to the last slave node inside } \\
\text { a master surface capture box along the } x \text {-coordinate }\end{array}$ \\
\hline$\left(i_{y}\right)_{\min }$ & $\begin{array}{l}\text { pointer into the Index vector corresponding to the first slave node } \\
\text { inside a master surface capture box along the y-coordinate }\end{array}$ \\
\hline$\left(\mathrm{i}_{\mathrm{y}}\right)_{\max }$ & $\begin{array}{l}\text { pointer into the Index vector corresponding to the last slave node inside } \\
\text { a master surface capture box along the } y \text {-coordinate }\end{array}$ \\
\hline$\left(i_{z}\right)_{\min }$ & $\begin{array}{l}\text { pointer into the Index vector corresponding to the first slave node } \\
\text { inside a master surface capture box along the } \mathrm{z} \text {-coordinate }\end{array}$ \\
\hline$\left(\mathrm{i}_{\mathrm{z}}\right)_{\max }$ & pointer into the Index vector corresponding to the last slave node inside \\
\hline Ibox & $\begin{array}{l}\text { a master surface capture box along the } z \text {-coordinate } \\
\text { number of slave nodes in each bucket }\end{array}$ \\
\hline & number of master nodes \\
\hline
\end{tabular}




\begin{tabular}{|c|c|}
\hline $\mathrm{m}_{\mathrm{s}}$ & number of master surfaces \\
\hline$\hat{\mathrm{m}}$ & unit normal of a master surface \\
\hline$\hat{\mathrm{m}}_{\mathrm{i}}$ & unit normal of master surface $i$ \\
\hline$\hat{\mathrm{m}}_{\mathrm{p}}$ & unit normal of master surface at end of previous time step \\
\hline $\overrightarrow{\mathrm{N}}_{\mathrm{m}}$ & surface normal of master surface \\
\hline n & number of slave nodes \\
\hline$\hat{\mathbf{n}}$ & unit normal of the slave surface at a slave node \\
\hline $\mathrm{n}_{\mathrm{b}}$ & total number of buckets \\
\hline nbox & bucket id for each slave node \\
\hline ndsort & list of slave nodes sorted by ascending bucket id \\
\hline npoint & $\begin{array}{l}\text { pointer into vector ndsort giving the starting location of the slave nodes } \\
\text { in each bucket }\end{array}$ \\
\hline$\hat{\mathrm{p}}_{\mathrm{t}}$ & unit push-back direction at time $\mathrm{t}$ \\
\hline $\mathrm{p}$ & penetration of slave node into master surface \\
\hline$R_{x}, R_{y}, R_{z}$ & Rank vector for the $\mathrm{x}, \mathrm{y}$, and $\mathrm{z}$ coordinate respectively \\
\hline$S_{x}, S_{y}, S_{z}$ & number of slices required along the $\mathrm{x}, \mathrm{y}$ and $\mathrm{z}$ coordinates respectively \\
\hline$S_{x}^{i}, S_{y}^{i}, S_{z}^{i}$ & slice along $x, y$ and $z$ coordinate containing node $i$ \\
\hline $\mathrm{t}$ & current time \\
\hline $\overrightarrow{\mathrm{V}}$ & velocity vector \\
\hline $\overrightarrow{\mathrm{V}}_{\mathrm{s}}$ & velocity of slave node \\
\hline $\overrightarrow{\mathrm{V}}_{\mathrm{m}}$ & velocity of master surface at contact point \\
\hline$v_{x}, v_{y}, v_{z}$ & $x, y$ and $z$ component of velocity \\
\hline$\left(v_{x}\right)_{\max }$ & maximum $x$-component of velocity \\
\hline$\left(v_{y}\right)_{\max }$ & maximum y-component of velocity \\
\hline$\left(v_{\mathrm{z}}\right)_{\max }$ & maximum z-component of velocity \\
\hline$x_{i}, y_{i}, z_{i}$ & $x, y$, and $z$ coordinate of master surface nodal point $i$ \\
\hline$x c_{\min }$ & minimum $x$-coordinate of master surface capture box \\
\hline$x c_{\max }$ & maximum x-coordinate of master surface capture box \\
\hline $\mathrm{yc}_{\min }$ & minimum y-coordinate of master surface capture box \\
\hline$y c_{\max }$ & maximum y-coordinate of master surface capture box \\
\hline$z c_{\text {min }}$ & minimum z-coordinate of master surface capture box \\
\hline $\mathrm{zc}_{\text {max }}$ & maximum z-coordinate of master surface capture box \\
\hline
\end{tabular}




\section{Table of Contents}

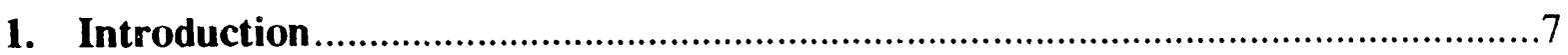

2. Survey of Contact Detection Algorithms and Motivation for Current Work ........8

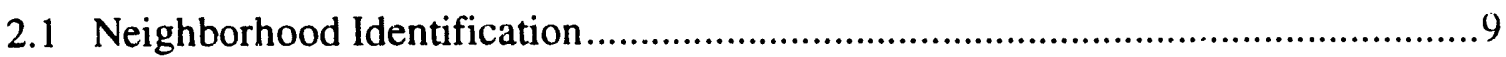

2.1.1 Surface Side-Set Pairing ........................................................................ 10

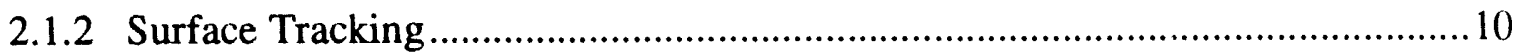

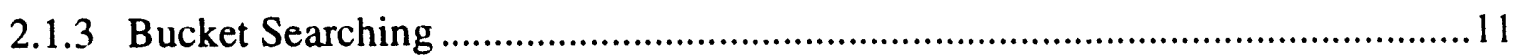

2.1.4 Pinball Contact....................................................................................12

2.2 Detailed Contact Check .............................................................................13

2.2.1 Ideal Contact Determination ......................................................................

2.2.2 Pushback inaccuracies .......................................................................... 14

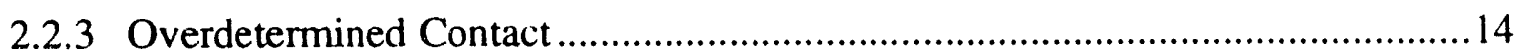

2.2.4 Undetermined Contact ...............................................................................16

2.3 Motivation for Current Work ..........................................................................17

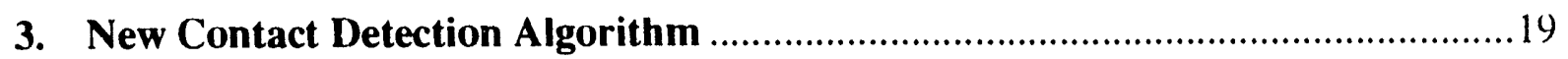

3.1 New Neighborhood Identification Strategy …….............................................19

3.1.1 Algorithm for vector architecture ………..................................................19

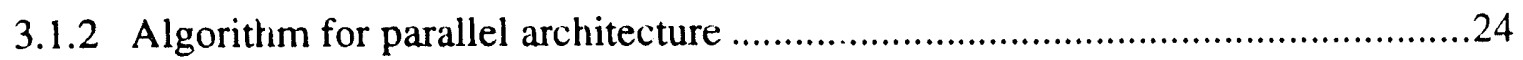

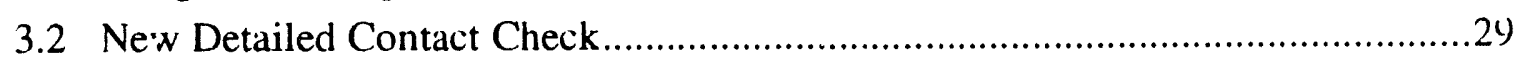

3.2.1 Velocity Based Contact Check ……………….............................................29

3.2.2 Static Contact Check ..................................................................................

3.3 Summary of New Contact Detection Algorithm .................................................33

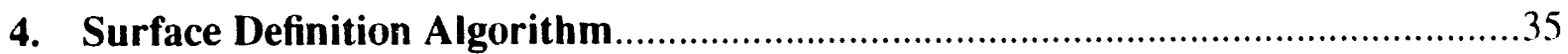

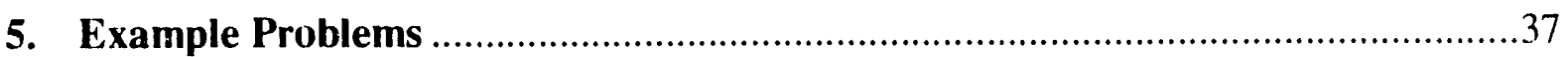

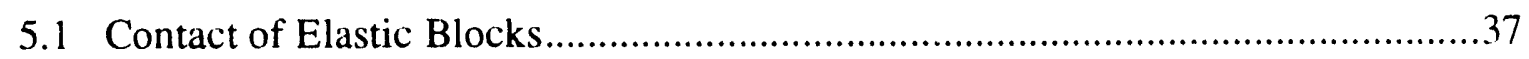

5.2 Contact Chatter under High Normal Loads:
Pressure Loading of Two Elastic Bodies

5.3 Self-Contacting Impact: Buckling of Shell-Like Structures...................................41

5.4 Automatic Contact Surface Redefinition: Cutting of a Steel Pipe...........................43

5.5 Multi-Body Impact: Elastic-Plastic Bar impacting Bricks .....................................46

5.6 Large Sliding Contact: Elastic-Plastic Forging of a Copper Billet.........................48

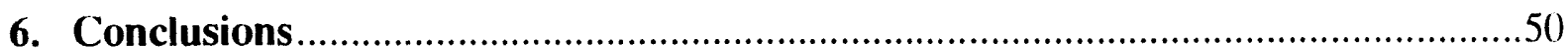

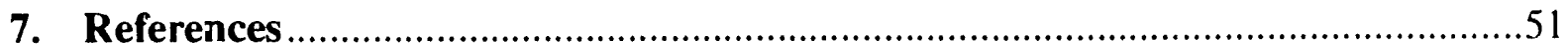

A1. Appendix 1: Derivation of velocity based contact check .......................................54

A2. Appendix 2: User instructions and example input files........................................60 


\section{List of Tables}

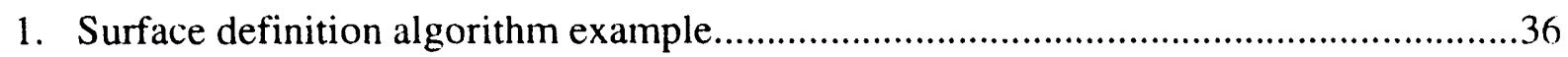




\section{List of Figures}

1. Master-slave relationship definition for contact enforcement ........................................9

2. Surface tracking and side set pairing required for self-contacting structures..................10

3. Detection of potential contacts for slave node 3 are influenced by bucket size ..............12

4. Calculation of the normal distance to contact point when contact is made......................13

5. Calculation of the normal distance to contact point as point slides on surface ................14

6. Resolving overdetermined contact by determining most opposed master surface ..........15

7. Determining contact point with consideration of slave node's movement.......................16

8. Resolving undetermined contact by identifying the closet point

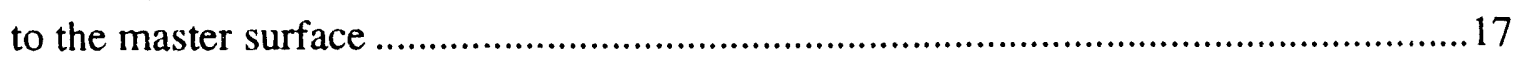

9. Slave node 2 tracking closest master node 14 results in a missed contact ......................18

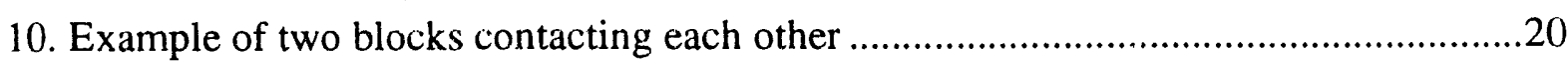

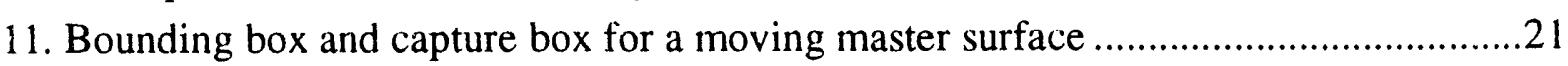

12. Remaining penetration due to a partially enforced contact constraint..........................22

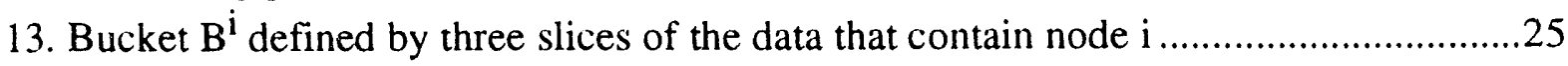

14. Master slave tracking using velocity and static contact check ......................................29

15. Initial estimates for the local coordinates of a contact point ..........................................

16. Push back direction for a concave surface based on minimum distance

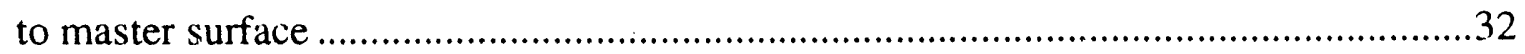

17. Push back direction for convex surface based on previous master surface normal........33

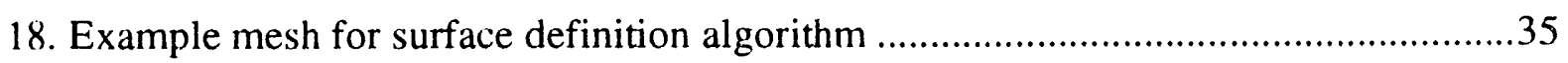

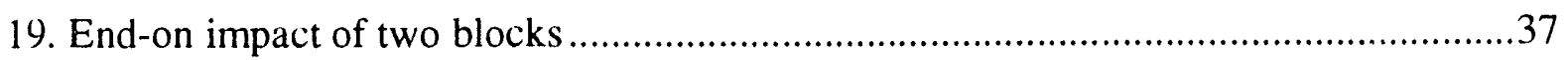

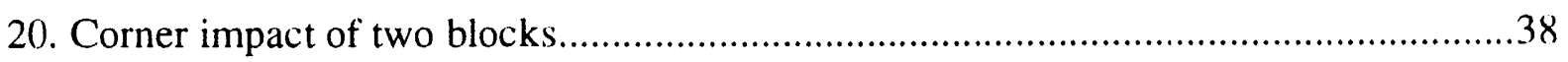

21. Two elastic bodies contacting under high normal load ...............................................39

22. Kinetic energy history and deformed shape (displacements magnified by $100 \mathrm{x}$ ) using old and new contact detection algorithm .........................................................

23. Finite element mesh of an elastic plate impacting an elastic-plastic can ......................41

24. Deformed meshes of the buckled can at various times...............................................42

25. Finite element model for simulating the cutting of a 2 inch steel pipe.........................43

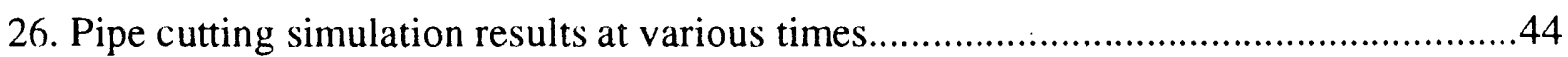

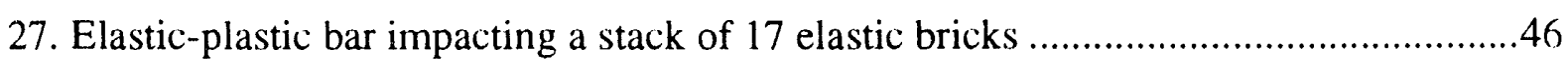

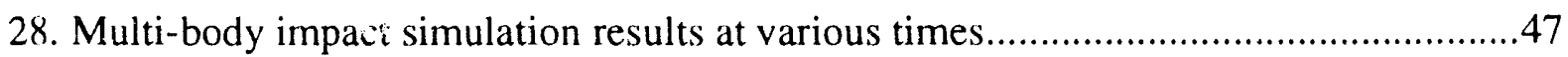

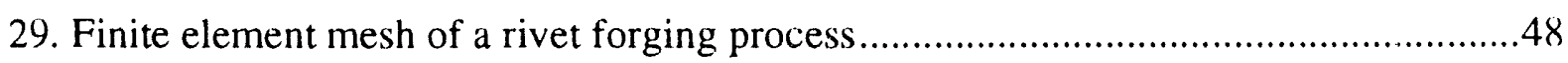

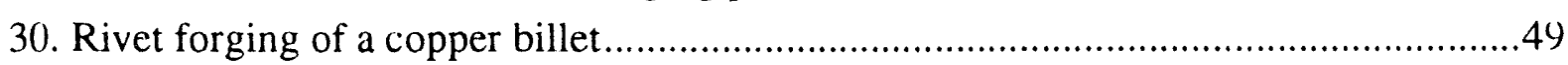

A 1.1. Triangular master surface definition on a quadrilateral element face.......................54

A1.2. Initial estimates for the local coordinates of a contact point ....................................58 


\section{Introduction}

An increasingly important aspect of large-scale finite element structural simulations is the efficient and accurate determination of contact between deformable bodies. At Sandia National Laboratories, the PRONTO [1][2][3] transient dynamics codes and the SANTOS [4] and JAC [5][6] quasistatic codes have been used to solve a wide variety of problems involving contact and other nonlinearities. However in some cases, the range of their applicability could be increased by improving the efficiency and accuracy of contact detection. These improvements would be beneficial in problems involving: structures that buckle and fold onto themselves; structures that have materials that tear and create new surfaces; multiple body contact/impact; and structures that slide relatively large distances over other surfaces. This report deals with one part of the contact problem, namely the detection of contact, which is distinct from the procedure used to enforce contact constraints. In these codes, which use a master-slave approach to contact problems, contact detection includes identifying the time, location, and amount of slave node penetration through some portion of a master surface.

The benefits of reducing the time spent on contact algorithms can be significant. For iterative equation solvers, such as those used in the Sandia codes, inaccuracies in the detection of contact lead to an increase in the number of iterations required for convergence. These inaccuracies arise mainly from incorrectly determining the location of contact as a slave node slides across another surface. For large finite element simulations with large numbers of slave nodes and master surfaces, as much as 50 percent of the total CPU time is spent using currently available contact algorithms. Thus, improvements in the speed and efficiency of contact detection could significantly reduce the total computational cost. More importantly, these improvements are also expected to allow one to solve problems which cannot be solved using existing algorithms.

This report reviews the current contact detection techniques used in the Sandia structural analysis codes and outlines the difficulties associated with these algorithms. A new algorithm is proposed that circumvents these difficulties. The key points of the new algorithm are that it:

i) uses a fast, memory-efficient global search to decide which slave nodes are in proximity to a master surface;

ii) does a detailed contact check using projected movements of both the master surface and slave node to determine the location, magnitude and direction of slave node penetration of the master surface; and

iii) automatically defines all surfaces given the mesh connectivity.

In Section 2 a short survey of the current contact detection algorithms and some difficulties associated with them is presented. Section 3 describes the proposed new contact detection algorithm, and in Section 4, an automatic surface definition algorithm is presented. Finally, in Section 5, example and verification problems using the new contact detection algorithm are discussed. Nomenclature used throughout the report is defined on the preceding pages. Two appendices are attached. In the first appendix, the velocity based contact check proposed in Section 3 is derived. The second appendix has a complete listing of all input files for the example problems presented in Section 5. 


\section{Survey of Contact Detection Algorithms and Motivation for Current Work}

The contact detection algorithms described in this section are typical for many finite element codes, including the transient dynamic codes PRONTO [1][2][3], DYNA[7][8], and ABAQUS Explicit [9], and the quasistatic codes SANTOS [4], JAC [5][6] and NIKE [10][11]. Most of this section specifically reviews the contact detection algorithms used in the PRONTO, SANTOS, and JAC codes. Difficulties associated with the contact detection algorithms are presented as a way for motivating the current work. A recent survey of the contact-impact algorithm in the DYNA codes was reported in [12].

Contact detection algorithms of interest here define a set of nodes called slave nodes and a set of surface patches called master surfaces. A siave node is simply a nodal point on the surface of the mesh. A master surface is defined using the side of a finite element on the surface. The surface topology is then simply a set of nodal points on the surface connected by straight line segments, corresponding to linear surface elements in 2D and bi-linear, quadrilateral surface elements in 3D. The master surface patches need not have the same order of interpolation as the finite element side that they represent. A four-node quadrilateral finite element side, for example, might be subdivided into four triangular master surfaces with the central node position being the average of the four corner nodes [13].

Contact detection is accomplished by monitoring the displacements of the slave nodes throughout the calculation for possible penetration of a master surface. Following contact detection, a contact constraint is defined so that the slave node is "pushed back" to remain on the master surface. Based on this description, it is convenient to separate contact algorithms into a location phase and a restoration phase. The location phase consists of a neighborhood identification and a detailed contact check. The neighborhood identification matches a slave node to a set of master surfaces that it potentially could contact. The detailed contact check determines which of the candidate master surfaces is in contact with a slave node, the point of contact, the amount of penetration, and the direction of push-back. The point of contact, amount of penetration, and the direction of pushback define a contact constraint that is then enforced in the contact enforcement or restoration phase of the contact algorithm. This constraint is enforced in the following time step or possibly over several time steps.

Although this report does not deal directly with contact enforcement, several aspects of it will affect the contact detection algorithm. The first is that the contact constraint is not necessarily enforced exactly in one time step for transient dynamics analyses, or in one iteration for quasistatic analyses. In part, this is due to the necessity of determining contact based on an approximate or projected configuration. In many cases, an estimated amount of slave node penetration is too low. Consequently, in the following time step (or iteration) the slave node is still penetrating the master surface. It is also likely and even desirable that the contact constraint intentionally be enforced in several time steps to improve the stability of the nonlinear equations of motion (or in several iterations to improve the convergence of integrating the nonlinear equilibrium equations in the case of quasistatic codes). This implies that the location phase must allow for the possibility that the contact constraint is not exactly enforced. 
Another aspect that will affect contact detection is that contact enforcement algorithms are based on a master-slave definition. For algorithms that require a strict master-slave treatment, the user must specify which surface is the master and which surface is the slave. This choice can have a significant effect on the resulting calculation and solution. For example, the coarser mesh should be designated as the master surface when the two contacting materials are the same, as shown in Figure 1a. If the master and slave surfaces are reversed as in Figure $1 \mathrm{~b}$, interpenetration that is not detected by the algorithm can result. The choice of masterslave roles is less clear when the materials of the contacting bodies are different. A more general contact enforcement approach requires that two passes be made through the contact detection algorithm, with master and slave surfaces exchanging roles. This two-pass approach, called a symmetric or partitioned contact algorithm, is more robust and often justifies the additional computational cost of the extra pass. The PRONTO codes also allow the user to specify a factor which partitions the master/slave roles of the contacting surfaces. For the contact detection algorithm, this implies that all nodes on the surfaces are slave nodes and all element faces on the surfaces are master surfaces.

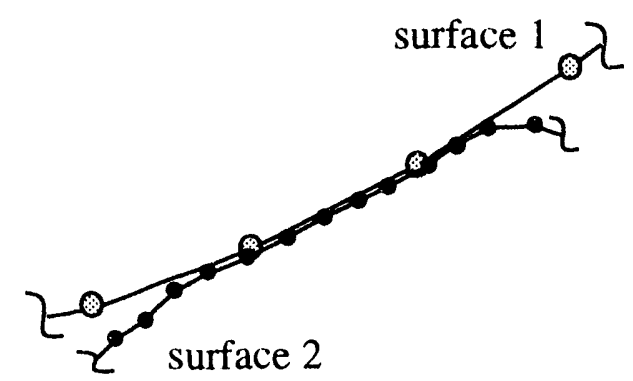

(a) surface 1 is the master and surface 2 is the slave

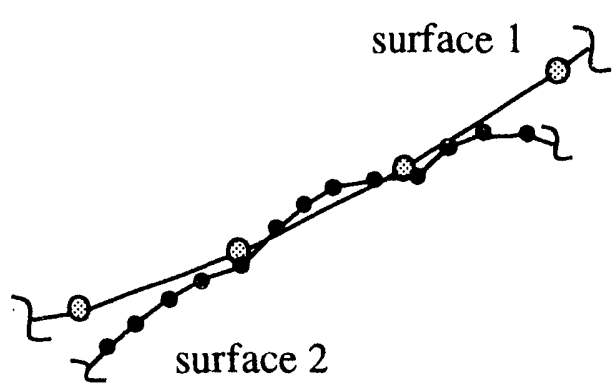

(b) surface 1 is the slave and surface 2 is the master

Figure 1. Master-slave relationship definition for contact enforcement.

\subsection{Neighborhood Identification}

Based on the description of contact detection, an algorithm called "neighborhood identification" is required to pair those slave nodes and master surfaces where potential contact is likely. The neighborhood identification phase is usually the most time consuming part of the contact detection algorithm. Obviously, the most robust approach would be to check every slave node against every master surface at every time step. Typically, the distances between each slave node and all master surface nodes are checked to find the closest master surface node. Master surfaces attached to this node are then considered as candidates for detailed contact checks. This exhaustive global searching approach requires nodal distance calculations on the order $n \times m$, where $n$ is the number of slave nodes on the surface and $m$ is the number of master nodes on the surface. Several algorithms have been proposed to speed up the neighborhood identification phase. These include surface side-set pairing, surface tracking, bucket searching, and pinball contact which are described below. 


\subsubsection{Surface Side-Set Pairing}

One simple and widely used approach to speed up neighborhood identification is to define subsets of the surface and prescribe pairs of subsets that may be in contact. Using these contact pairs, a search may be restricted to only those slave nodes and master nodes which are included in the two subsets. All of the Sandia codes currently use surface side set pairing. A recent extension of this idea subdivides the contact side sets further and has been developed and implemented in DYNA3D [14]. This approach is effective when the contact surface pair has a very small number of slave nodes and master surfaces. For larger problems faster search techniques are needed.

\subsubsection{Surface Tracking}

To speed up the neighborhood identification further, some codes including PRONTO use a process called surface tracking $[1,2,6,7]$. Surface tracking requires the definition of contact surface pairs but does not require an exhaustive $n x m$ search between all slave nodes and master nodes. The surface tracking algorithms currently in PRONTO and DYNA are based on two assumptions regarding the behavior of contacting surfaces. First, the spatially' closest master surface node is assumed to be attached to the master surface that the slave node contacts. This assumption allows a very simple distance calculation to find what is called the tracked master surface node. Once the tracked master surface node is known, a detailed contact check for each master surface connected to the node can be made. The second assumption is that from one time step to another, the new tracked master surface node can be found near the currently-tracked node. This assumption allows the tracking to be updated by a local search for the nearest master surface node starting with the currently-tracked node and moving radially outward. To avoid searching for neighboring master surface nodes during the calculation, the tracking algorithm constructs a list of master surface neighbors at the start of the calculation and stores it for future reference. This requires a considerable amount of memory but is usually justified because a significant amount of computing time can be saved.

A surface tracking algorithm based on these assumptions cannot effectively model two classes of problems. The first is a structure buckling and folding onto itself as shown in Figure 2.

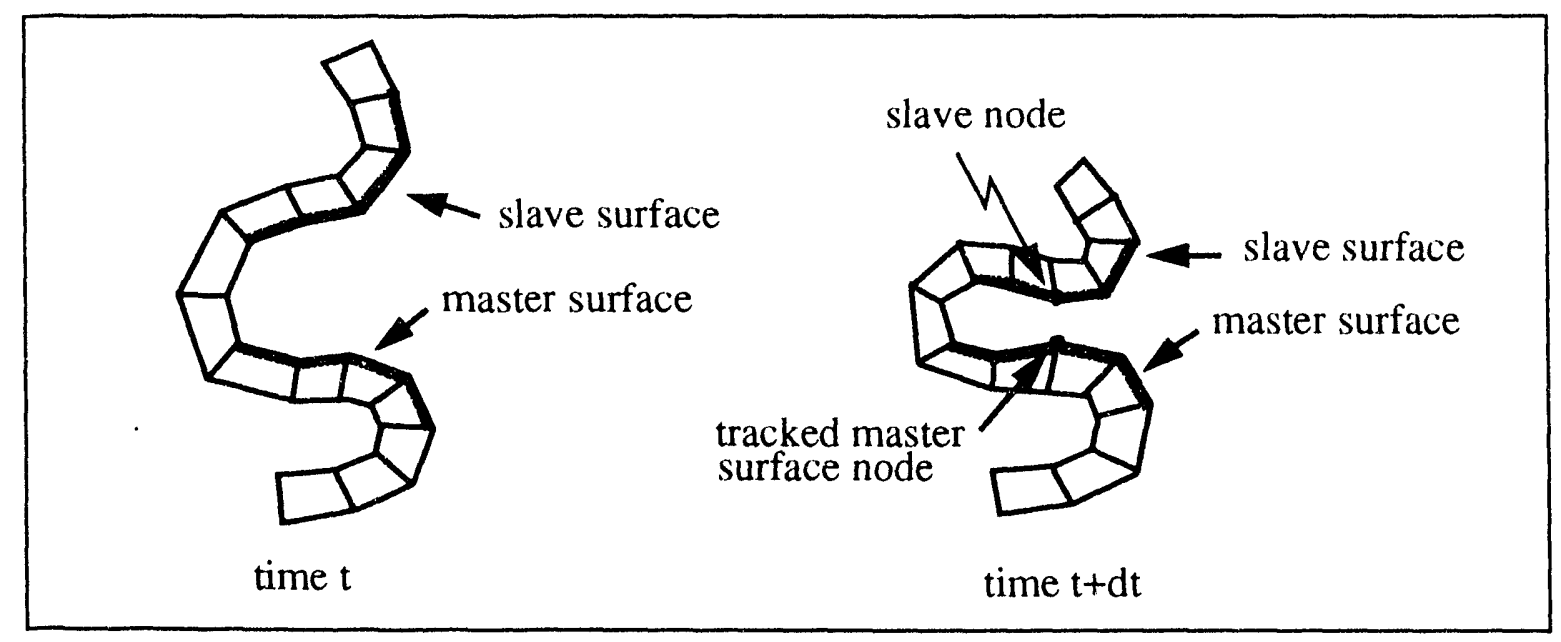

Figure 2. Surface tracking and side set pairing required for self-contacting structures 
Since the portions of the surface that come into contact are not known a priori, the contact surface definition and pairing must be done intermittently throughout the analysis [15]. This extensive interaction by the user makes the solution of self contact difficult. The second class of problems involves structures with surfaces that tear or erode. Usually the newly created surface finds itself in contact with other parts of the structure. The fixed data structure required for surface tracking is an obstacle for redefining the surface and allowing further contact.

\subsubsection{Bucket Searching}

Recently several algorithms using a bucket search have been proposed for modelling self contacting surfaces [12][16]. These global search algorithms are referred to as bucket searches because the domain of the problem is broken down into buckets (or boxes) into which the slave nodes and master nodes are sarted. The domain of the proble $n$ is first subdivided into $S_{x}$ slices along the $x$-coordinate, $S_{y}$ slices along the $y$-coordinate, and $S_{z}$ slices along the $z$ coordinate. The intersection of any three orthogonal slices defines a bucket. Potential interactions between nodes are determined by looping through the buckets and collecting the slave nodes and master nodes in neighboring buckets. Typically, a closest master node is determined for each slave node using a distance check. Assuming a uniform distribution of $n+m$ slave and master nodes throughout the domain, the total number of distance comparisons for a $3 \mathrm{D}$ problem (reported in [12]) is:

$$
\mathrm{C}_{3 \mathrm{D}}=(n+m)\left[\frac{27(n+m)}{\left(\mathrm{S}_{\mathrm{x}} \mathrm{S}_{\mathrm{y}} \mathrm{S}_{\mathrm{z}}\right)}-1\right]
$$

After the distance check, all master surfaces connected to the closest master node are then considered for potential contact with the slave node. This method for determining potential interactions implies that the bucket size should be chosen based on the largest master surface dimension to avoid missing a potential contact, as shown in Figure 3 . The small bucket size in Figure 3 a results in a missed contact because the master nodes 7 and 8 are not in the neighboring buckets of slave node 3 . The larger bucket size in Figure $3 \mathrm{~b}$ results in detecting the correct contact of slave node 3 with the master surface defined by master nodes 7 and 8 . Based on this observation, a contact problem involving very dissimilar mesh sizes requires large buckets compared to many of the elements. Consequently, a large number of potential interactions may be found in each bucket.

A limitation of the bucket searching algorithm is the potential need for a large amount of memory. At first glance this algorithm appears to require $k S_{x} S_{y} S_{z}$ memory locations, where $k$ is the maximum number of nodes likely to be found in a bucket. With some attention to memory management, these requirements can be significantly reduced for certain problems. For other problems the large amount of memory required remains an obstacle. If the problem domain expands greatly, either the number of buckets must increase (to maintain the same resolution) or the bucket size must increase (to maintain the same amount of memory). Either one adversely effects the bucket search efficiency. This dependency on the bucket size or, equivalently, on the geometry of the problem domain is considered as a limitation of current bucket searching techniques. 


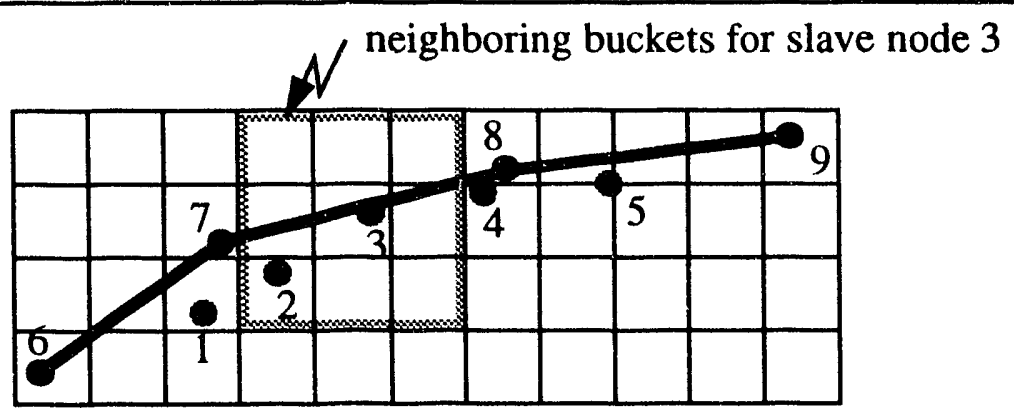

slave node master node

(a) a small bucket size results in a missed contact

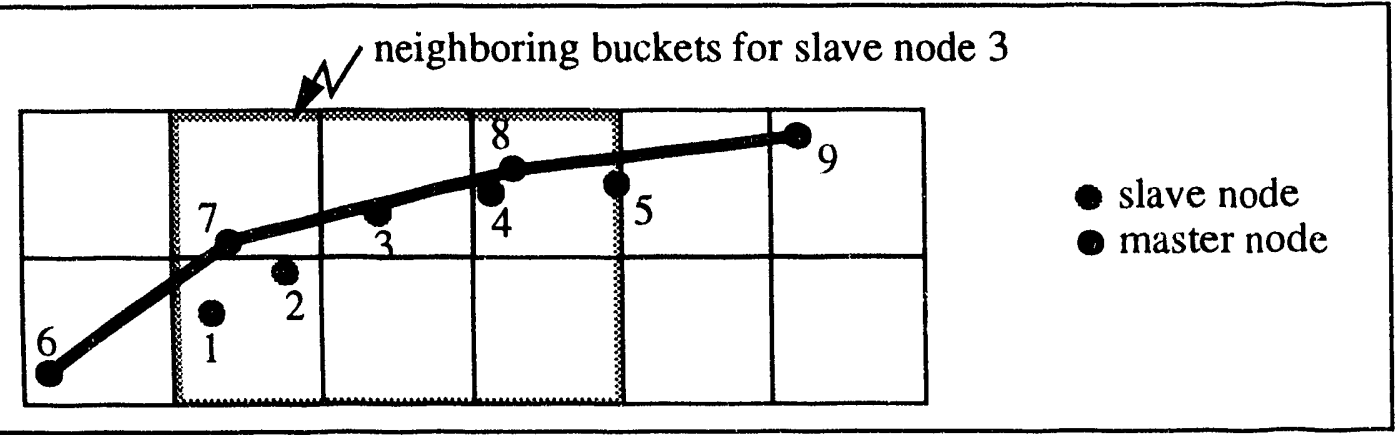

(b) a large bucket size results in detecting correct contact

Figure 3. Detection of potential contacts for slave node 3 are influenced by bucket size

Even with these possible difficulties, the distinct advantage that bucket searching algorithms have is that they lend themselves to a parallel architecture, as discussed by Plimpton [20].

\subsubsection{Pinball Contact}

Another contact detection algorithm that uses a global search was proposed by Belytschko [17]. It is referred to as pinball contact since a circle is inscribed within each element on the surface of a two-dimensional mesh. (A sphere is inscribed within each element on the surface of a three-dimensional mesh). Potential contact is detected simply by overlap of any two spheres. The procedure vectorizes and therefore is suited to large-scale 3D computations where fast and efficient algorithms are imperative. To aid this overlap detection, a sorting or searching technique, such as bucket searching, may be necessary. After pinball overlap is detected, a more detailed contact check can be used to obtain a more accurate prediction of the contact constraint. The method was intended for problems where impact is the primary form of contact. Two dimensional [18] and three dimensional [17] problems have been successfully solved using the pinball algorithm. There is, however, a class of problems where the pinball algorithm does not work well. For problems where sliding contact and friction is encountered, the resolution of the mesh near the surface has a dramatic effect on the accurate prediction of contact forces. A mesh with varying resolution at the surface gives a poor prediction of contact pressures and forces. This is a result of modelling a geometrically flat surface with a series of inscribed circles and is a limitation of the pinball contact algorithm. 


\subsection{Detailed Contact Check}

After gathering a list of potential interactions using any one of the searching techniques described above, a detailed contact check is done for each slave node - master surface pair. The following section presents what is typically done in a detailed contact check. This check autermines: (1) which of the candidate master surfaces is in contact with the slave node, (2) the point of contact, (3) the amount of penetration, and (4) the direction the slave node should be pushed back. This overview is presented using $2 \mathrm{D}$ surfaces composed of lines for simplicity, but the theory applies equally to 3D surfaces composed of bilinear patches.

\subsubsection{Ideal Contact Determination}

The ideal condition for determining contact is shown in Figure 4. The slave node is unambiguously outside a master surface at time $t$ and inside the same surface at time $t+d t$. At time $t+d t$, the distance, $p$, from the slave node to the master surface is calculated by projecting the vector $\vec{d}_{m s}$ onto the surface normal $\hat{m}_{l}$ :

$$
\mathrm{p}=\left|\overrightarrow{\mathrm{d}}_{\mathrm{ms}} \bullet \hat{\mathrm{m}}_{1}\right|
$$

The vector $\vec{d}_{m s}$ is defined, as shown in Figure 4, from the master surface node $i$ to the slave node at time $t+d t$ :

$$
\vec{d}_{m s}=\left[x_{s}(t+d t)-x_{i}(t+d t)\right] \grave{i}+\left[y_{s}(t+d t)-y_{i}(t+d t)\right] \grave{j}
$$

where $\left(x_{s}(t+d t), y_{s}(t+d t)\right)$ and $\left(x_{i}(t+d t), y_{i}(t+d t)\right)$ are the coordinates of the slave node and master surface node $i$ at time $t+d t$, respectively. This normal distance, $p$, is called the amount of penetration and the unit vector $\hat{p}_{t}=\hat{m}_{l}$ is called the pushback direction, where $\hat{p}_{t}$ is defined to pass through the slave node at time $t+d t$. The contact point, $x$, is determined by intersecting the master surface with the pushback direction:

$$
\left(x_{c}, y_{c}\right)=\left(x_{s}, y_{s}\right)+\left(p\left(\hat{p}_{t} \bullet \dot{i}\right), p\left(\hat{p}_{t} \bullet \hat{j}\right)\right)
$$

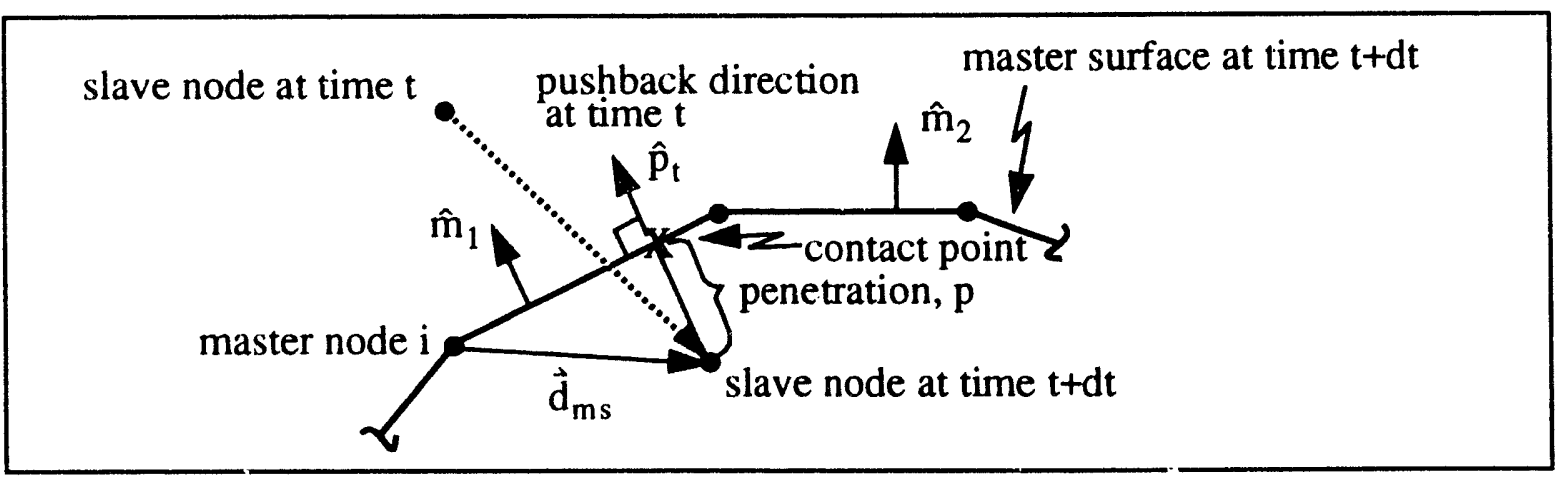

Figure 4. Calculation of the normal distance to contact point when contact is made 


\subsubsection{Pushback inaccuracies}

Unfortunately, there are many ambiguous cases of contact that arise because of the discretization of the surface. The surface is discretized as a collection of $\mathrm{C}_{0}$ continuous finite element sides in 2D (or faces in $3 \mathrm{D}$ ). $\mathrm{C}_{0}$ continuity in the geometric interpolation results in a surface normal that is not continuous. This can have adverse effects on the accuracy of the contact enforcement. Consider, for example, the case where a slave node is sliding across the surface shown in Figure 5. One must keep in mind that the enforcement of the contact constraint is not exact in one time step and could be enforced over several time steps. It is possible then, that the slave node be penetrating the master surface at time $t+d t$ (a time step $d t$ after contact was detected). As the slave node slides from being in contact with master surface 1 to being in contact with master surface 2 , the pushback direction is changing to coincide with the normal of master surface 2 . This change in the pushback direction artificially introduces added slave node motion along its trajectory. This is a direct result of the inexact enforcement of the contact constraint in one time step. In calculations where friction is modelled, this \%elocity can add noise to the solution.

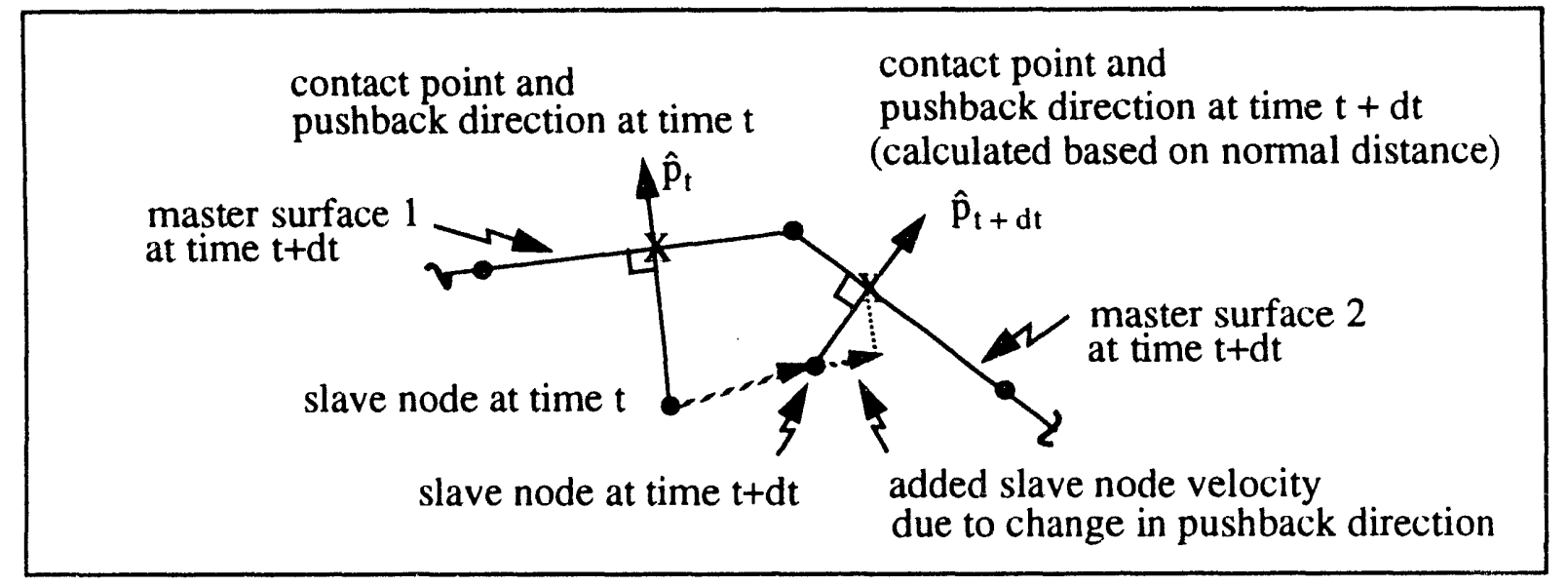

Figure 5. Calculation of the normal distance to contact point as point slides on surface

\subsubsection{Overdetermined Contact}

Further ambiguity is introduced when the contact algorithm performs the detailed contact checks based only on the estimated (deformed) configuration. For example, Figure 6 shows two different scenarios where a slave node can make contact with two master surfaces. Since the slave node motion is not used to determine how contact was made at time $t+d t$, two contact constraints can be defined (one with master surface 1 and the other with master surface 2). The algorithm, then, must resolve the overdetermined contact and decide which contact constraint to enforce. This is commonly done by determining which master surface is most opposed to the slave node surface normal [1][7][9]. The surface normal $\hat{n}$ at a slave node is defined as the average of the normals of all the element faces connected to the slave node. A master surface is most opposed to the slave surface normal when the quantity $|\hat{n} \bullet \hat{m}|$ is largest. It is common to assume that the most opposed master surface is the one on which the 
contact constraint should be enforced, so that in Figure 6a, the slave node is contacting master surface 1 and in Figure $6 \mathrm{~b}$ the slave node is contacting master surface 2. The most opposed criterion is often called the strength of contact check.

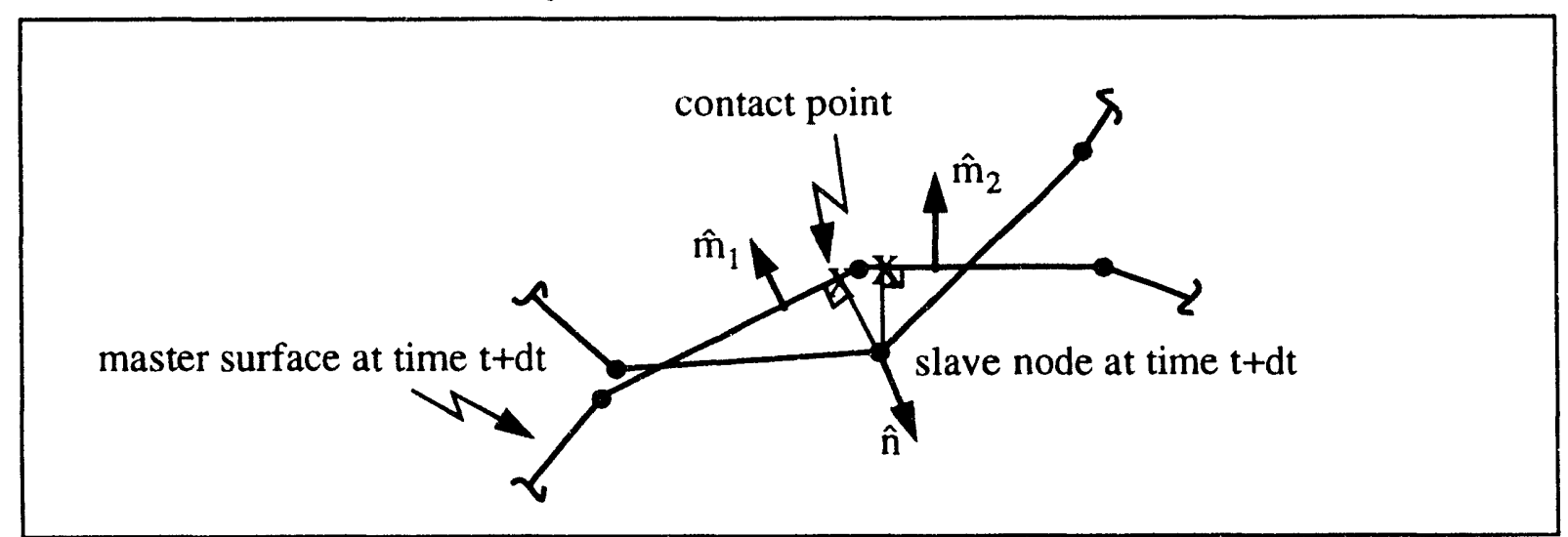

(a) slave node more opposed to master surface 1: $\left|\hat{n} \bullet \hat{m}_{1}\right|>\left|\hat{n} \bullet \hat{m}_{2}\right|$

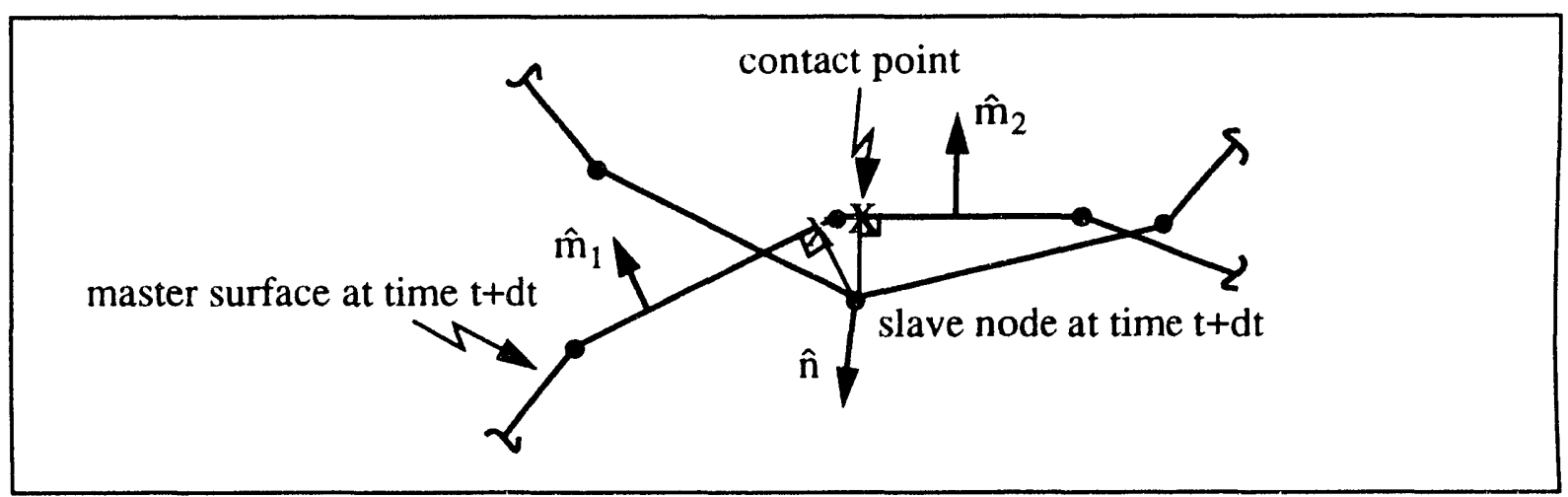

(b) slave node more opposed to master surface 2 : $\left|\hat{\mathrm{n}} \bullet \hat{\mathrm{m}}_{2}\right|>\left|\hat{\mathrm{n}} \bullet \hat{\mathrm{m}}_{1}\right|$

Figure 6. Resolving overdetermined contact by determining most opposed master surface.

When two surfaces are already in contact the strength of contact check can be effective. However, when the surfaces are just coming into contact, as shown in Figure 7, this type of static contact check cannot consistently resolve the overdetermined contact and predict the correct contact constraint. In Figure 7a, the slave node makes contact with master surface 1. In Figure $7 \mathrm{~b}$, the slave node makes contact with master surface 2 . The contacts are a result of the motion of the slave node and are not determined by the master surface which is most opposed. 


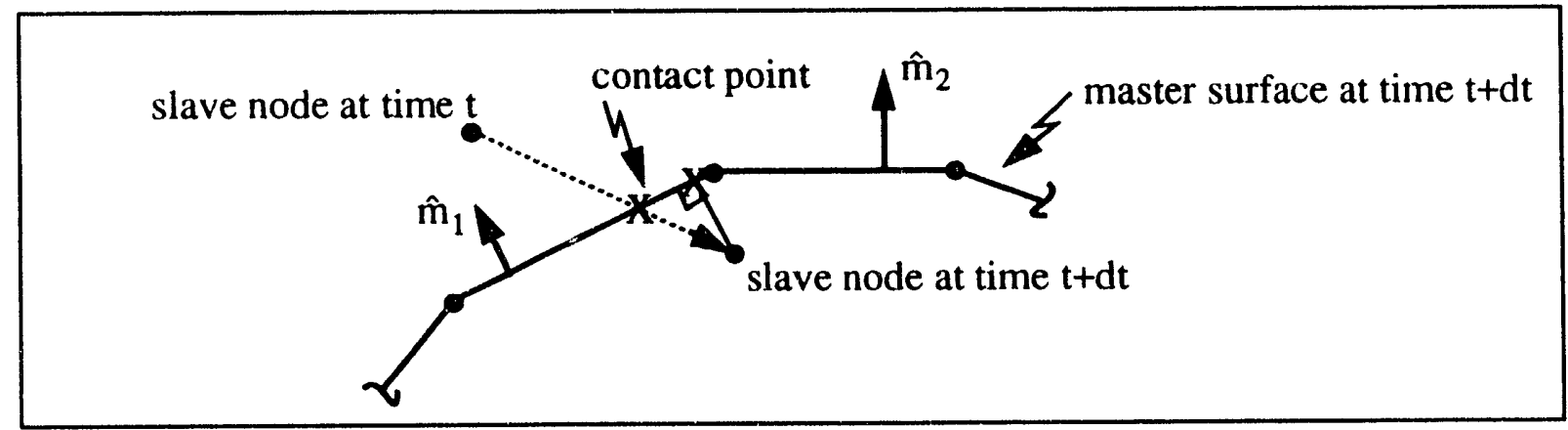

(a) contact point on master surface 1

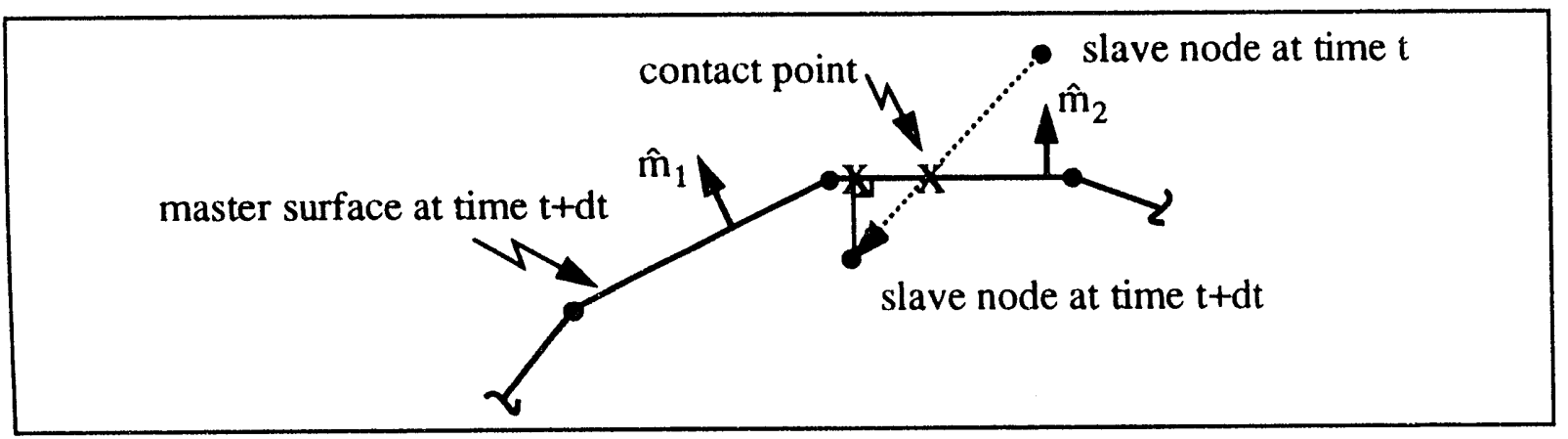

(b) contact point on master surface 2

Figure 7. Determining contact point with consideration of slave node's movement.

\subsubsection{Undetermined Contact}

Another ambiguity exists when the normal distance is undetermined. Figure 8 illustrates a situation when this can occur. The normal distance measured from the slave node to the master surface does not intersect any master segment. Intersection implies that the contact point, $x$, on the master surface lies between the master surface nodes, and not beyond them as shown in Figure $8 \mathrm{a}$ and $\mathrm{b}$. Current algorithms typically allow an extension, $\varepsilon$, of the master surface so that contact can be detected. This extension means that either one contact is found or, in other cases, both contacts are detected and a choice between the two must be made. With either case, the contact point is still incorrectly defined. This inaccurate determination of the contact point causes "contact chatter", (see example 5.2).

In this situation, the detailed contact check should identify the vertex of the two master surfaces as the contact point since it is the closest point on the master surface, as shown in Figure 8c. 


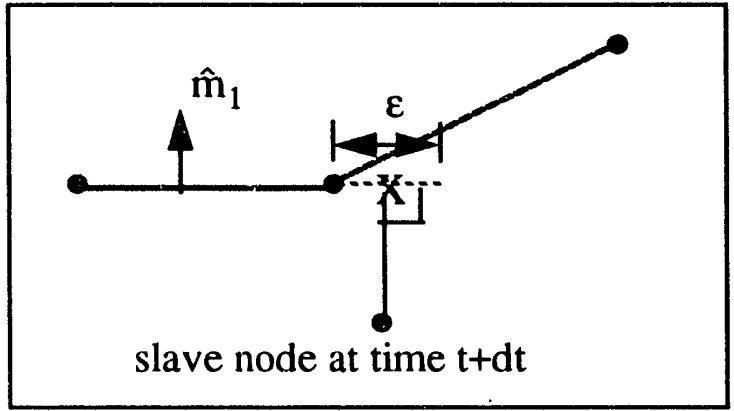

(a) contact with master surface 1

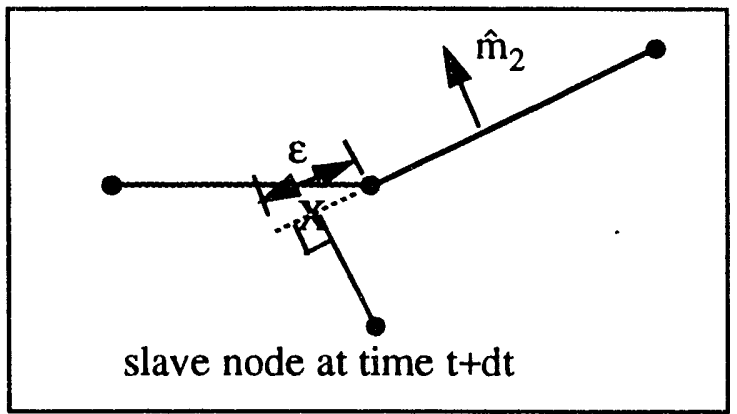

(b) contact with master surface 2

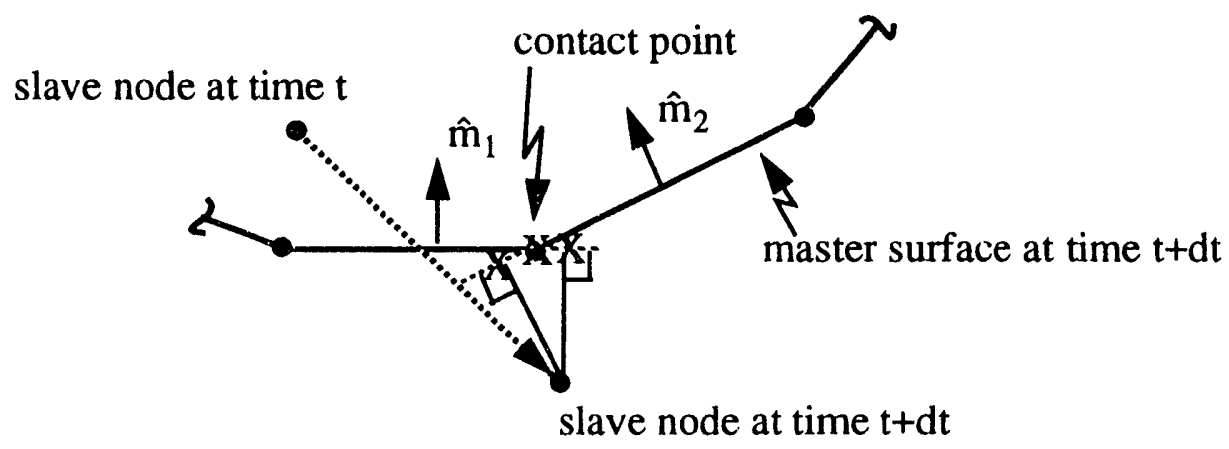

(c) contact at vertex of master surface 1 and master surface 2

Figure 8. Resolving undetermined contact by identifying the closet point to the master surface

\subsection{Motivation for Current Work}

Several algorithms for efficient neighborhood identification have been summarized in this section. These included surface side-set pairing, surface tracking, bucket searching, and pinball contact. This current technology works successfully for a large variety of contact problems. There are some problems, however, where improvements in neighborhood identification and searching are required. Improvements would be beneficial in problems involving: structures that buckle and fold onto themseives; structures that have materials that tear and create new surfaces; multiple body contact/impact; and structures that slide relatively large distances over other surfaces.

In particular, improvements could address two distinct concerns with current search techniques. One concern is that nearly all neighborhood identification techniques are for nodes. For example, the result of a search is always the closest master node for a given slave node (with one exception in pinball overlap). A slave node does not always contact a master surface connected to the closest master node. Figure 9 shows a very simple shell self-contact 
example demonstrating this fact. The slave node 2 is tracking master node 14 , however it is actually contacting master surface 8-9 (defined by surface nodes 8-9). The tracked master node 14 implies that a detailed contact check would consider master surfaces 13-14 and 14-15 only and not master surface 8-9 as it should. Contact is always made between a slave node and a master surface, and an improved neighborhood identification should reflect this.

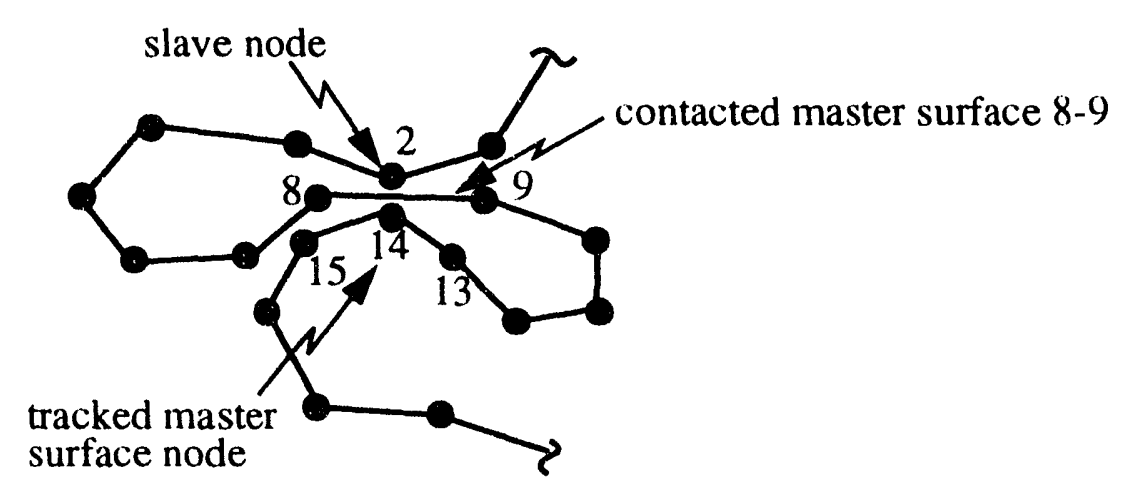

Figure 9. Slave node 2 tracking closest master node 14 results in a missed contact

Another concern is that current global sorting and searching routines depend on the problem domain. The efficiency of bucket searching is adversely affected by problems that grow or expand in spatial dimension. Significant improvements in the speed and efficiency of the search phase could be made if it depended only on the number of master surfaces and slave nodes in the problem, and not on the problem geometry.

The detailed contact check was also summarized in this section. The difficulties associated with detailed contact determination included (1) inaccurate pushback direction, (2) overdetermined (multiply defined) potential contact, and (3) undetermined contact. An improved contact detection algorithm should address these issues and offer improved accuracy in determining the contact point, penetration, and pushback direction. 


\section{New Contact Detection Algorithm}

The proposed contact detection algorithm outlined in the following section offers improvements in the efficiency and accuracy of contact detection. The improvements are the result of a neighborhood identification strategy that uses a global contact search and an accurate detailed contact check that uses the projected motion of both master and slave surfaces. The algorithm does not require contact surface pairing and, therefore, easily handles self-contacting surfaces, eroding and tearing surfaces, and random contact between multiple bodies. The algorithm also resolves the ambiguities that arise because of the surface discretization and from using only the deformed configuration for detailed contact checks.

\subsection{New Neighborhood Identification Strategy}

The proposed neighborhood identification strategy uses a global search to determine what slave nodes are in close proximity to a master surface. It accumulates these potential interactions by constructing a local neighborhood around a master surface and globally searching for all slave nodes that are in the neighborhood. Two global search algorithms are presented, one that takes advantage of the characteristics of vector processors, and one that takes advantage of the characteristics of parallel processing. The efficiencies of each search algorithm are still under investigation. Both algorithms have been implemented on computers with scalar and vector processors. The algorithm for vector architecture uses a new search routine developed by Swegle [19]. The algorithm for parallel architecture is an extension of the state-of-the-art bucket searching technique [12], with a few significant exceptions.

To illustrate the two search algorithms, the example shown in Figure 10 of two blocks contacting each other will be used. Each block is a cube with dimensions 1"x 1"x l". The centroid of block 1 is initially at the coordinates $(0.5$ ", 0.5 ", 0.5 ") and is moving with a velocity $v_{y}=500 \mathrm{in} / \mathrm{s}$ in the $y$-direction. The stable time step is approximately $1.1 \times 10^{-6} \mathrm{~s}$ so that block 1 moves approximately $5.5 \times 10^{-4}$ inches during one time step. Block 2 is initially at rest with its centroid located at the coordinates $(0.5 ", 1.5 ", 0.5$ "). The symmetric contact enforcement being used implies that every node and element face on the surface is included in the contact surface. There are 52 slave nodes $(n=52)$ and 48 master surfaces $\left(m_{s}=48\right)$.

\subsubsection{Algorithm for vector architecture}

A search algorithm using $7 n$ memory locations and requiring on the order of $m_{s} \log _{2} n$ comparisons is utilized for the global location strategy. The algorithm is based on a particle search technique developed by Swegle [19]. It sorts the slave nodes by location and uses a binary search to construct a list of slave nodes that are in a master surface neighborhood. The search algorithm implemented here depends only on the number of slave nodes $n$ and not on the geometry of the problem. It takes advantage of the known positions of the slave nodes and master surfaces as well as their predicted positions in the next time step.

The slave nodes are sorted based on their current coordinates, with each coordinate sorted independently. This sorting is done using an index vector so that the order of the slave nodes listed in the index vector corresponds to their coordinate value, with coordinates ordered from smallest to largest. For the current example, there are 52 slave nodes numbered as shown in Figure 10, resulting in the following index vectors: 


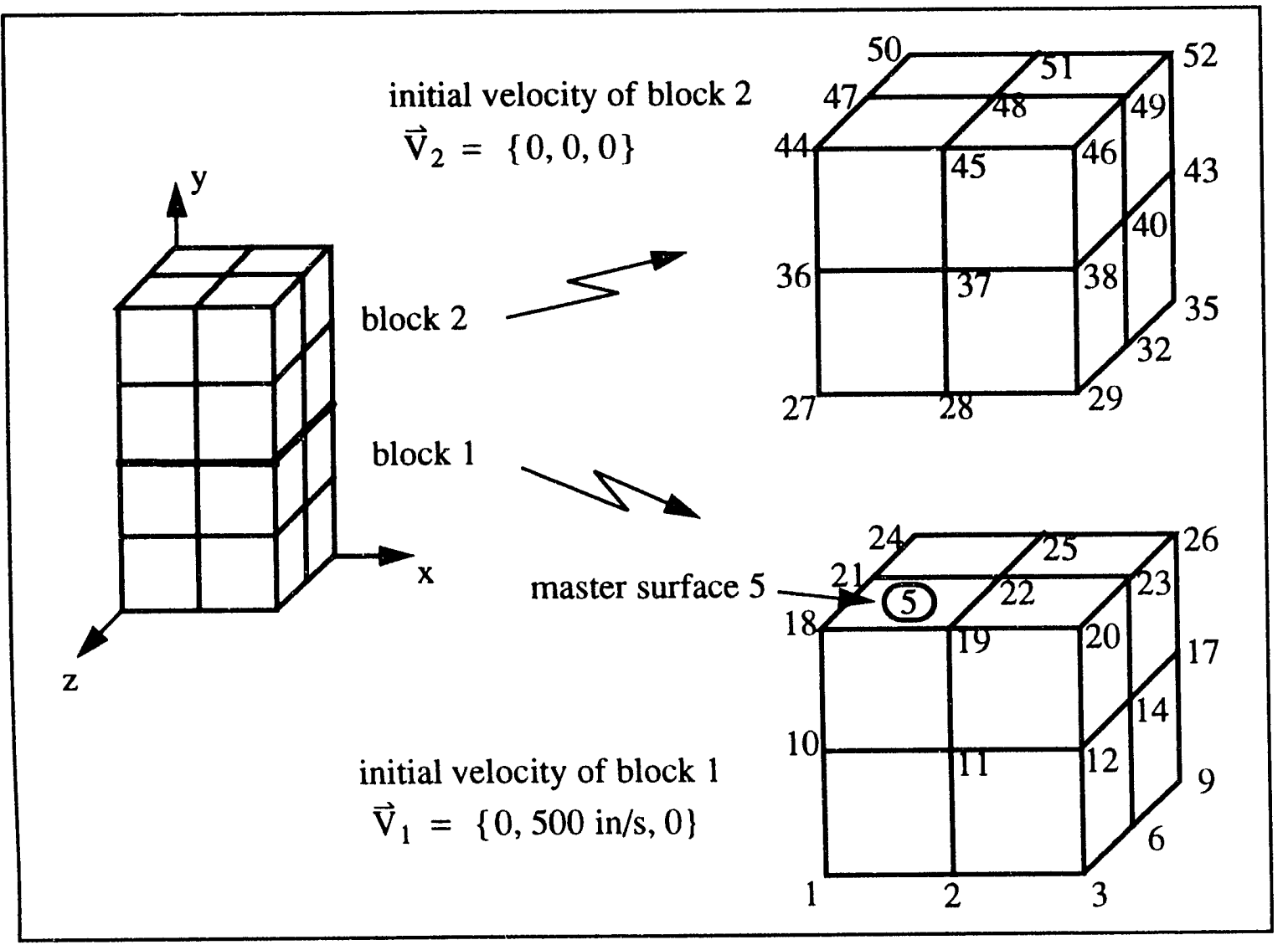

Figure 10. Example of two blocks contacting each other.

$$
\begin{aligned}
& I_{x}=\{1,4,7,10,13,15,18,21,24,27,30,33,36,39,41,44,47,50,2,5,8,11,16,19 \text {, } \\
& 22,25,28,31,34,37,42,45,48,51,3,6,9,12,14,17,20,23,26,29,32,35,38 \text {, } \\
& 40,43,46,49,52\} \\
& \begin{aligned}
& I_{y}=\{1,2,3,4,5,6,7,8,9,10,11,12,13,14,15,16,17,18,19,20,21,22,23,24,25,26 \\
& 27,28,29,30,31,32,33,34,35,36,37,38,39,40,41,42,43,44,45,46,47,48 \\
&49,50,51,52\}
\end{aligned} \\
& I_{z}=\{7,8,9,15,16,17,24,25,26,33,34,35,41,42,43,50,51,52,4,5,6,13,14,21 \text {, } \\
& 22,23,30,31,32,39,40,47,48,49,1,2,3,10,11,12,18,19,20,27,28,29,36 \text {, } \\
& 37,38,44,45,46\}
\end{aligned}
$$

for the $\mathrm{x}, \mathrm{y}$, and $\mathrm{z}$ coordinates respectively. A rank vector is also constructed for the slave nodes. It gives the location of each slave node in the index vector and is required to avoid searching the index vector for a given slave node. It can be easily constructed by looping through the index vector. For example, suppose a slave node $i$ is stored at position $j$ in the index vector. The rank vector would then store the pointer $j$ at its position $i$. For the current example, the rank vectors are: 


$$
\begin{aligned}
& R_{x}=(1,19,35,2,20,36,3,21,37,4,22,38,5,39,6,23,40,7,24,41,8,25,42,9,26,43 \text {, } \\
& 10,27,44,11,28,45,12,29,46,13,30,47,14,48,15,31,49,16,32,50,17,33 \text {, } \\
& 51,18,34,52\} \\
& R_{y}=\{1,2,3,4,5,6,7,8,9,10,11,12,13,14,15,16,17,18,19,20,21,22,23,24,25,26 \text {, } \\
& 27,28,29,30,31,32,33,34,35,36,37,38,39,40,41,42,43,44,45,46,47,48 \text {, } \\
& 49,50,51,52\} \\
& R_{Z}=\{35,36,37,19,20,21,1,2,3,38,39,40,22,23,4,5,6,41,42,43,24,25,26,7,8,9 \text {, } \\
& 44,45,46,27,28,29,10,11,12,47,48,49,30,31,13,14,15,50,51,52,32,33 \text {, } \\
& 34,16,17,18\}
\end{aligned}
$$

After the slave nodes are sorted by $\mathrm{x}, \mathrm{y}$ and $\mathrm{z}$ coordinate, the master surfaces are processed sequentially. This processing involves defining a local neighborhood for each master surface by bounding the space occupied by a master surface at its known location at time $t$ and its predicted location at time $t+d t$. Figure 11 shows a bounding box for a master surface over one time step. Another box, called the capture box is also constructed to collect slave nodes that potentially contact the master surface.

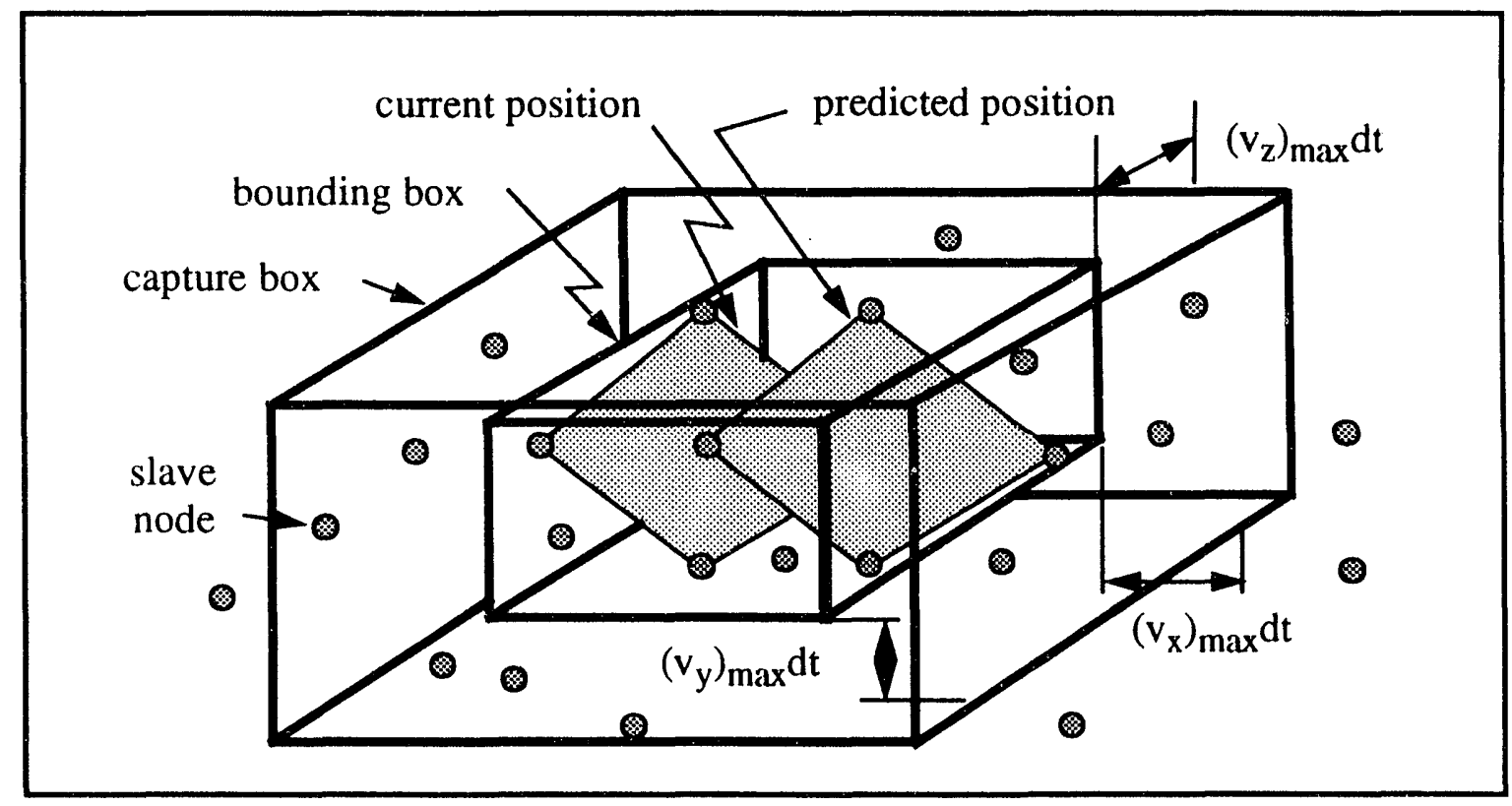

Figure 11. Bounding box and capture box for a moving master surface

For example, suppose the maximum distance any slave node moves in one time step is $\left(v_{x}\right)_{\max } d t$ in the $x$-direction. Then a slave node within a distance $\left(v_{x}\right)_{\max } d t$ from the bounding box could potentially contact the master surface. This holds for the $y$ - and z-directions as well so that a capture box can be constructed, as shown in Figure 11. Any slave node inside the capture box should therefore be considered for potential contact with the master surface. In the example problem, $\left(\mathrm{v}_{\mathrm{x}}\right)_{\max }=0,\left(\mathrm{v}_{\mathrm{y}}\right)_{\max }=500 \mathrm{in} / \mathrm{s},\left(\mathrm{v}_{\mathrm{z}}\right)_{\max }=0$, and $\mathrm{dt}=1.1 \times 10^{-6} \mathrm{~s}$, so the capture box for master surface 5 would have the corners: 


$$
\begin{aligned}
& x_{\min }=0.0 \text { in., } x_{\max }=0.5 \text { in. } \\
& y_{\min }=1.0-v_{y} d t=0.99945 \text { in. }, \quad y_{\max }=1.0+v_{y} d t=1.00055 \text { in. } \\
& z_{\min }=0.5 \text { in. }, z_{\max }=1.0 \text { in. }
\end{aligned}
$$

At this point it is necessary to address one aspect of the contact enforcement algorithm that will affect contact detection. Recalling that a contact constraint is likely to be enforced over several time steps, the capture box must be enlarged to capture slave nodes with a partially enforced contact constraint. A distance, $\mathrm{p}_{\mathrm{r}}$, equivalent to the amount of penetration not enforced is easily computed for each slave node in contact with a master surface, as shown in Figure 12. The capture box is enlarged in all directions by this distance, $\mathrm{p}_{\mathrm{r}}$.

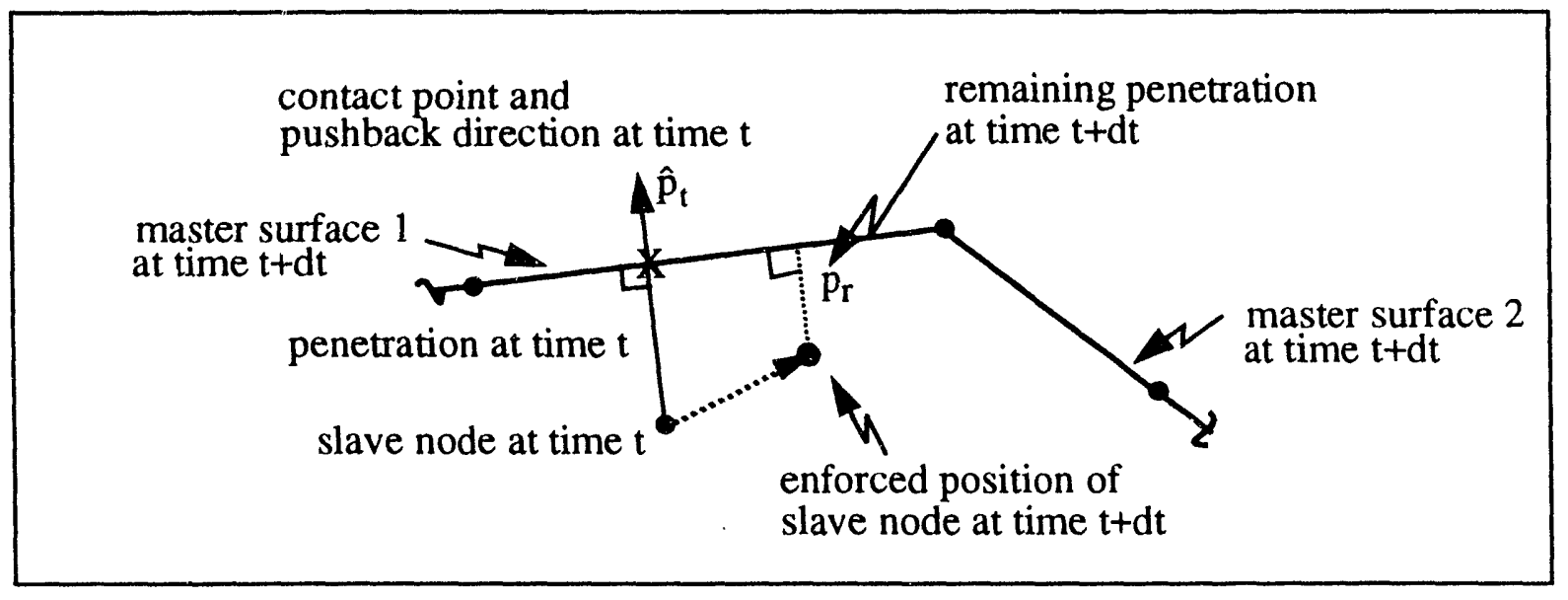

Figure 12. Remaining penetration due to a partially enforced contact constraint

After defining the capture box a binary search is done on the index vectors to find the slave nodes that are inside the capture box. The binary search results in two pointers for each coordinate direction. One is a lower pointer into the index vector that corresponds to the position in the index vector of the first slave node inside the capture box and the other is an upper pointer that corresponds to the position in the index vector of the last slave node inside the capture box.

For the example problem node 1 at position 1 in $\mathrm{I}_{\mathrm{x}}$ is the first node in the box in the $\mathrm{x}$ direction, and node 51 at position 34 in $I_{x}$ is the last node inside the capture box. In the $y$ direction node 18 in position 18 of $\mathrm{I}_{\mathrm{y}}$ and node 35 at position 35 in $\mathrm{I}_{\mathrm{y}}$ are the first and last nodes just inside the capture box, respectively. Similarly, in the $z$ direction node 4 in position 19 of $I_{z}$ and node 46 at position 52 in $I_{z}$ are the first and last nodes inside the capture hox, respectively. Therefore, the binary search would give the following results:

$$
\begin{aligned}
& \left(i_{x}\right)_{\min }=1,\left(i_{x}\right)_{\max }=34 \\
& \left(i_{y}\right)_{\min }=18,\left(i_{y}\right)_{\max }=35
\end{aligned}
$$




$$
\left(\mathrm{i}_{\mathrm{z}}\right)_{\min }=19,\left(\mathrm{i}_{\mathrm{z}}\right)_{\max }=52
$$

This means, for example, that the subset of slave nodes from $I_{x}\left(\left(i_{x}\right)_{\min }\right)$ to $I_{x}\left(\left(i_{x}\right)_{\max }\right)$ are in the capture box of the master surface on the basis of the $x$-coordinate. For the example problem, the subset or list of slave nodes for each coordinate direction that are in the capture box of master surface 5 are:

$$
\begin{aligned}
& \mathrm{L}_{\mathrm{x}}=\{1,4,7,10,13,15,18,21,24,27,30,33,36,39,41,44,47,50,2,5,8,11,16,19,22 \text {, } \\
& 25,28,31,34,37,42,45,48,51\} \\
& L_{y}=\{18,19,20,21,22,23,24,25,26,27,28,29,30,31,32,33,34,35\} \\
& \mathrm{L}_{\mathrm{z}}=\{4,5,6,13,14,21,22,23,30,31,32,39,40,47,48,49,1,2,3,10,11,12,18,19,20 \text {, } \\
& 27,28,29,36,37,38,44,45,46\}
\end{aligned}
$$

Finally, a contraction is done to find the slave nodes in the capture box of the master surface in all three coordinate directions simultaneously. To accomplish this, each of the slave nodes in one list is selected and then checked if its rank is between the lower and upper pointer in the other two coordinates. For computational efficiency the shortest list of slave nodes is selected, which can be determined by selecting the smallest of $\left[\left(i_{w}\right)_{\max }-\left(i_{w}\right)_{\min }+1\right], w=x, y$, or $z$. For the example problem, $L_{y}$ is the list with the smallest number of slave nodes, so that slave nodes $i=\mathrm{I}_{\mathrm{y}}\left(\left(\mathrm{i}_{\mathrm{y}}\right)_{\min }\right), \quad \mathrm{I}_{\mathrm{y}}\left(\left(\mathrm{i}_{\mathrm{y}}\right)_{\min }+1\right), \ldots, \mathrm{I}_{\mathrm{y}}\left(\left(\mathrm{i}_{\mathrm{y}}\right)_{\max }\right)$ are in the capture box if $\left(\mathrm{i}_{\mathrm{x}}\right)_{\min } \leq \mathrm{R}_{\mathrm{x}}(i) \leq\left(\mathrm{i}_{\mathrm{x}}\right)_{\max }$ and $\left(\mathrm{i}_{\mathrm{z}}\right)_{\min } \leq \mathrm{R}_{\mathrm{z}}(i) \leq\left(\mathrm{i}_{\mathrm{z}}\right)_{\max }$.

To help illustrate this procedure, the first few slave nodes in list $\mathrm{L}_{\mathrm{y}}$ are processed as follows:

1) $i=\mathrm{I}_{\mathrm{y}}\left(\left(\mathrm{i}_{\mathrm{y}}\right)_{\min }\right)=18, \mathrm{R}_{\mathrm{x}}(18)=7$, and $\mathrm{R}_{\mathrm{z}}(18)=41$.

Since $\left(i_{x}\right)_{\min } \leq 7 \leq\left(i_{x}\right)_{\max }$ and $\left(i_{z}\right)_{\min } \leq 41 \leq\left(i_{z}\right)_{\max }$ slave node 18 is in the capture box of master surface 5 .

2) $i=\mathrm{I}_{\mathrm{y}}\left(\left(\mathrm{i}_{\mathrm{y}}\right)_{\min }+1\right)=19, \mathrm{R}_{\mathrm{x}}(\mathrm{i} 9)=24$, and $\mathrm{R}_{\mathrm{z}}(19)=42$.

Since $\left(\mathrm{i}_{\mathrm{x}}\right)_{\min } \leq 24 \leq\left(\mathrm{i}_{\mathrm{x}}\right)_{\operatorname{ma}}$ and $\left(\mathrm{i}_{\mathrm{z}}\right)_{\min } \leq 42 \leq\left(\mathrm{i}_{\mathrm{z}}\right)_{\max }$ slave node 19 is also in the capture box of master surface 5 .

3) $i=\mathrm{I}_{\mathrm{y}}\left(\left(\mathrm{i}_{\mathrm{y}}\right)_{\min }+2\right)=20, \mathrm{R}_{\mathrm{x}}(20)=41$, and $\mathrm{R}_{\mathrm{z}}(20)=43$.

Since $41>\left(i_{x}\right)_{\max }$ slave node 20 is NOT in the capture box of master surface 5 .

After processing all of the slave nodes in list $\mathrm{L}_{\mathrm{y}}$, it is found that only the slave nodes $\{18,19,21,22,27,28,30,31\}$ are within the bounding box of master surface 5 .

For efficiency on a vector computer each slave node-master surface pair is stored in an array 
for later processing after a vector length of pairs is accumulated. Note that only those pairs are added where the slave node is not also a node on the master surface. For the example problem only the following four pairs would be added to the array:

$$
\{(27,5),(28,5),(30,5) \text { and }(31,5)\}
$$

At this point an exhaustive search has been done and no assumptions have been made on the manner in which contact can or will be made. The processing that will determine contact is called the detailed contact check. In the current implementation of the algorithm, the binary sorting is implemented every time step.

\subsubsection{Algorithm for parallel architecture}

The following algorithm is essentially a conventional bucket search [12] with two significant exceptions. These exceptions are that (1) the bucket size $b_{s}$ is based on the smallest master surface dimension and (2) a capture box is used to ensure all potential contacts are gathered for a detailed contact check. The capture box takes advantage of the known positions of the slave nodes and master surfaces as well as the predicted positions in the next time step. Both are described in detailed after the bucket search algorithm is reviewed.

The bucket search algorithm first sorts the slave nodes into buckets, then finds the buckets that a master surface occupies and pairs the master surface with the slave nodes in those buckets. It requires $(18 n)$ memory locations plus two vectors with lengths equal to the number of buckets $\left(n_{b}\right)$.

To sort the slave nodes into buckets, an integer coordinate system is constructed in each physical direction. Assuming that the bucket size $b_{s}$ is based on the dimension of the smallest master surface, the number of slices (each with thickness $b_{s}$ ) required in the $x, y$, and $z$ directions are:

$$
\begin{aligned}
& S_{x}=\operatorname{int}\left[\left(x_{\max }-x_{\min }\right) / b_{s}\right]+1 \\
& S_{y}=\operatorname{int}\left[\left(y_{\max }-y_{\text {min }}\right) / b_{s}\right]+1 \\
& S_{z}=\operatorname{int}\left[\left(z_{\text {max }}-z_{\text {min }}\right) / b_{s}\right]+1
\end{aligned}
$$

The three slices containing any given node, $\mathrm{i}$, can be calculated as follows:

$$
\begin{aligned}
& S_{x}^{i}=\operatorname{int}\left[\left(x_{i}-x_{\min }\right) / b_{s}\right]+1 \\
& S_{y}^{i}=\operatorname{int}\left[\left(y_{i}-y_{\min }\right) / b_{s}\right]+1 \\
& S_{z}^{i}=\operatorname{int}\left[\left(z_{i}-z_{\min }\right) / b_{s}\right]+1
\end{aligned}
$$

The intersection of the three orthogonal slices defines the bucket $B^{i}$ containing node $i$, as shown in Figure 14. 


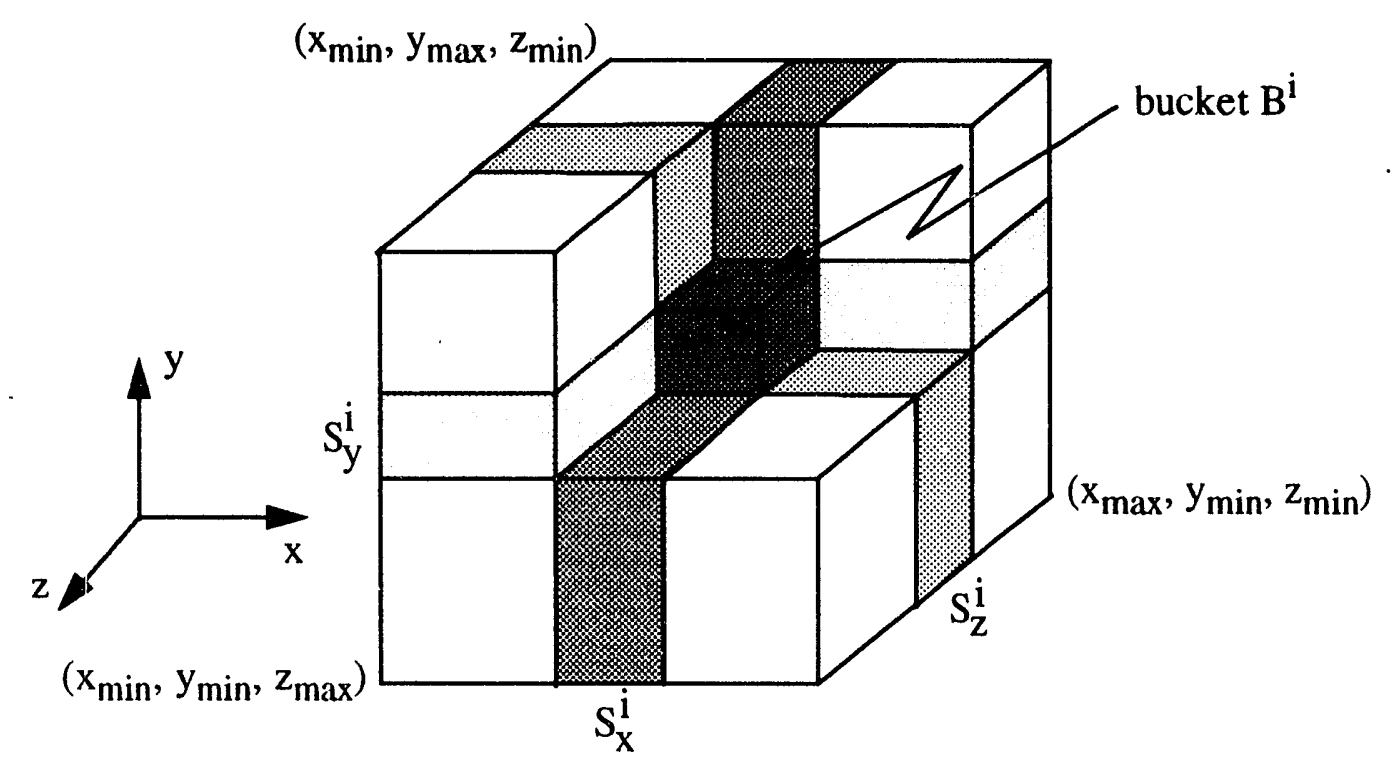

Figure 13. Bucket $B^{i}$ defined by three slices of the data that contain node $i$.

If buckets are numbered sequentially, progressing first in the $x$ direction, next in the $y$ direction, and then in the $\mathrm{z}$ direction, the bucket id containing node $\mathrm{i}$ is given by;

$$
B^{i}=\left(B_{z}^{i}-1\right) S_{x} S_{y}+\left(B_{y}^{i}-1\right) S_{x}+B_{x}^{i}
$$

With the eventual aim of efficiently determining a list of slave nodes within any given bucket, two vectors of length $n$ are created. One stores the bucket id for each slave node, lbox, and the other is a list of slave nodes sorted by bucket id, ndsort. Two additional vectors of length $n_{b}$ are also constructed to efficiently access the slave nodes. The first contains the number of slave nodes occurring in each bucket, nbox, and the other is a pointer that identifies the first slave node in each bucket, npoint. The entire sorting procedure can be summarized as follows:

1. Zero the vector (nbox) containing the number of nodes in each bucket.

2. Find the bucket id $\left(B^{i}\right)$ for each node.

3. Store the bucket id for node $i$ in the vector: $l b o x(i)=B^{i}$

4. Increment the counter for bucket $B^{i}: \operatorname{nbox}\left(B^{i}\right)=\operatorname{nbox}\left(B^{i}\right)+1$

5. Calculate the pointer for each bucket $j$ into a sorted list of nodes.

$$
n \operatorname{npoint}(I)=1 \quad n \operatorname{noint}(j)=n \operatorname{noint}(j-1)+n \operatorname{nbox}(j-1)
$$

6. Zero the vector nbox 
7. Sort the slave nodes according to their bucket number into a list ndsort.

$$
\begin{aligned}
& n d \operatorname{sort}(\operatorname{nbox}(\operatorname{lbox}(i))+\operatorname{npoint}(\operatorname{lbox}(i)))=i \\
& \operatorname{nbox}(\operatorname{lbox}(i))=\operatorname{nbox}(\operatorname{lbox}(i))+I
\end{aligned}
$$

For the example problem, the bucket size is 0.5 inches corresponding to the dimension of the smallest master surface. This implies that $S_{x}=3, S_{y}=5$, and $S_{z}=3$ and a total number of buckets $n_{b}=45$. The bucket sort of all 52 slave nodes would give the following results:

Bucket id for each slave node:

$$
\begin{aligned}
\operatorname{lbox}= & \{31,32,33,16,17,18,1,2,3,34,35,36,19,21,4,5,6,37,38,39,22,23,24,7,8,9 \\
& 37,38,39,22,23,24,7,8,9,40,41,42,25,27,10,11,12,43,44,45,28,29,30 \\
& 13,14,15\}
\end{aligned}
$$

Pointer into ndsort giving the starting location of the slave nodes in each bucket:

$$
\begin{aligned}
\text { npoint }= & \{1,2,3,4,5,6,7,9,11,13,14,15,16,17,18,19,20,21,22,23,23,24,26,28,30,31, \\
& 31,32,33,34,35,36,37,38,39,40,41,43,45,47,48,49,50,51,52\}
\end{aligned}
$$

List of slave nodes sorted by their bucket id:

$$
\begin{aligned}
\text { ndsort }= & \{7,8,9,15,16,17,24,33,25,34,26,35,41,42,43,50,51,52,4,5,6,13,14,21, \\
& 30,22,31,23,32,39,40,47,48,49,1,2,3,10,11,12,18,27,19,28,20,29,36,37 \\
& 38,44,45,46\}
\end{aligned}
$$

Number of slave nodes in each bucket:

$$
\begin{aligned}
\text { nbox }= & \{1,1,1,1,1,1,2,2,2,1,1,1,1,1,1,1,1,1,1,0,1,2,2,2,1,0,1,1,1,1,1,1,1,1,1,1,2,2,2, \\
& 1,1,1,1,1,1\}
\end{aligned}
$$

To illustrate how all slave nodes within, for example, bucket 23 are gathered, the sorted information described above is used as follows. The number of slave nodes in bucket 23 is given by: $\operatorname{nbox}(23)=2$. The slave nodes occupying bucket 23 can be found starting at location npoint(23) $=26$ in ndsort. This implies that slave nodes at location 26 and 27 in ndsort are in bucket 23: $\operatorname{ndsort}(26-27)=$ slave nodes $\{22,31\}$.

The sorting algorithm above is identical to that described in [12][16]. Potential interactions between nodes in [12][16] are determined by looping through the buckets and collecting the slave nodes in neighboring buckets, using the pointer into the sorted list. This implies that the bucket size must be based on the largest master surface dimension to avoid missing potential contact, as was demonstrated in Figure 3.

To avoid these difficulties, the strategy that the proposed search algorithm employs is to collect all the buckets occupied by a master surface. Then, using the information obtained during sorting, the slave nodes in those buckets are collected. This ensures that all potential 
interactions with a given master surface are found regardless of bucket size (and therefore problem geometry). In the current algorithm, the bucket size should be interpreted as the sorting refinement instead of a length measure of the largest master surface. A bucket size based on the smallest master surface assures that there will be a small number of slave nodes in each bucket and admits the possibility that a master surface could occupy many buckets.

The collection of slave nodes with which a master surface could potentially interact is determined in the following way:

1. A capture box is constructed for each master surface, as described previously

2. The buckets containing the extreme corners of the master surface capture box are determined

$$
\begin{aligned}
& \text { ibox }_{\min }=\min \left(S_{x}, \text { ifix }\left(\left(x c_{\min }-x_{\min }\right) / b_{s}\right)+1\right) \\
& \text { jbox }_{\min }=\min \left(S_{y}, \text { ifix }\left(\left(y c_{\min }-y_{\min }\right) / b_{s}\right)+1\right) \\
& \operatorname{kbox}_{\min }=\operatorname{inin}\left(S_{z}, \text { ifix }\left(\left(z c_{\min }-z_{\min }\right) / b_{s}\right)+1\right) \\
& \text { ibox }_{\max }=\min \left(S_{x}, \text { ifix }\left(\left(x c_{\max }-x_{\min }\right) / b_{s}\right)+1\right) \\
& \text { jbux }_{\max }=\min \left(S_{y}, \text { ifix }\left(\left(y c_{\max }-y_{\min }\right) / b_{s}\right)+1\right) \\
& \operatorname{kbox}_{\max }=\min \left(S_{z}, \text { ifix }\left(\left(z c_{\max }-z_{\min }\right) / b_{s}\right)+1\right)
\end{aligned}
$$

wherc $x c_{\min }$ and $x c_{\max }$ are the corners of the master surface capture box in the $x$ direction $\left(\mathrm{yc}_{\min }, \mathrm{yc}_{\max }, \mathrm{zc}_{\min }\right.$ and $\mathrm{zc} \mathrm{c}_{\max }$ have the same definitions in the $\mathrm{y}$ an $\mathrm{l} \mathrm{z}$ directions, respectively).

3. All buckets within the ranges calculated in step 2 are identified as follows:

$$
\begin{aligned}
& \text { Loop from ibox }=\text { ibox }_{\min } \text { to } \text { ibox }_{\max } \\
& \text { Loop from jbox }=\text { jbox }_{\min } \text { to } \text { jbox }_{\max } \\
& \text { Loop from kbox }=\mathrm{kbox}_{\min } \text { to } \mathrm{kbox}_{\max } \\
& \mathrm{B}^{\mathrm{i}}=(\mathrm{kbox}-1) \mathrm{S}_{\mathrm{x}} \mathrm{S}_{\mathrm{y}}+(\mathrm{jbox}-1) \mathrm{S}_{\mathrm{x}}+\mathrm{ibox}
\end{aligned}
$$

\section{Endloop}

\section{Endloop}

\section{Endloop}

4. All slave nodes in the buckets calculated in step 3 are considered for potential interaction with the master surface

In this approach, the algorithm takes advantage of the observation that the buckets that a niaster surface occujies can be easily determined. In the example problem, assume that master surface 5 is to be processed. The corners of the capture box of master surface 5 are: 


$$
\begin{aligned}
& x c_{\min }=0.0 \text { in., } \quad x c_{\max }=0.5 \text { in. } \\
& y c_{\min }=0.99945 \text { in. }, \quad y c_{\max }=1.00055 \text { in. } \\
& z c_{\min }=0.5 \text { in., } \quad z c_{\max }=1.0 \text { in. }
\end{aligned}
$$

so that the capture box would occupy the range of buckets:

$$
\begin{aligned}
& \text { ibox }_{\min }=1, \text { ibox }_{\max }=2 \\
& \text { jbox }_{\min }=3, \text { jbox }_{\max }=3 \\
& \operatorname{kbox}_{\min }=2, \operatorname{kbox}_{\max }=3
\end{aligned}
$$

This identifies buckets $22,23,37$, and 38 . The slave nodes occupying these buckets would be considered for potential contact with master surface 5 . Note that only the slave nodes which are not also nodes on master surface 5 are considered, so that only the following four pairs would be added to the vectorized list:

$$
\{(27,5),(28,5),(30,5) \text { and }(31,5)\}
$$

In the current implementation of the algorithm, the bucket sorting is implemented every time step in a vectorized mode. A study of the efficiencies of a bucket search algorithm was presented by Plimpton [20] for a computer with parallel architecture. For a certain class of spatially compact problems, the bucket searching seems to be faster than the vectorized global search. Further speedup might be anticipated by sorting every 5 to 10 time steps, suggested by [12], while storing the nearest neighbors for contact determination in the intermediate time steps. 


\subsection{New Detailed Contact Check}

The proposed detailed contact check distinguishes between slave nodes that are not in contact and those that are already in contact. It does so to resolve the ambiguities that arise in each case. The ambiguity shown in Figure 7, for example, could be easily resolved by considering the velocity of the slave node. This idea of a velocity based contact check can be extended to include a moving slave node contacting a moving master surface, as shown in Figure.14a. For slave nodes just coming into contact the velocity based contact check identifies the point of contact (or impact) and the contact time. Figure 14b shows a static contact check that is used for slave nodes already in contact with a master surface. For these slave nodes, ambiguities arise because the surface normal is not continuous. This can result in not finding any contact when there should be one or finding multiple solutions to a single contact. The proposed velocity based and static based detailed contact checks resolve these ambiguities, as shown in the following sections.

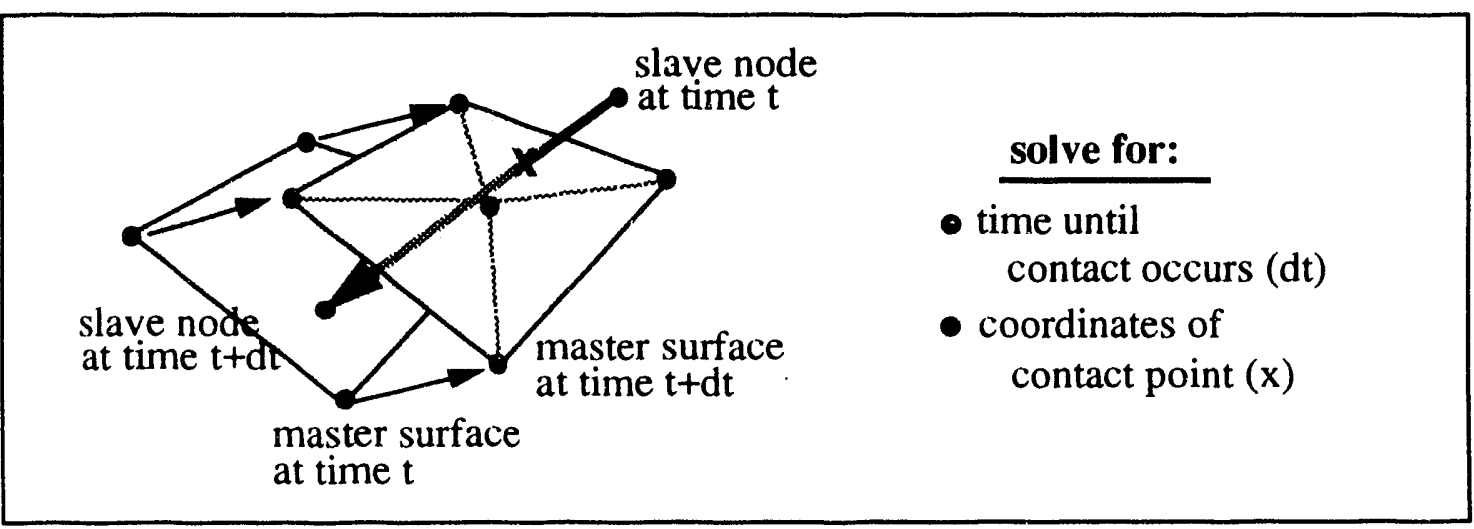

(a) velocity based contact check based on position and velocity

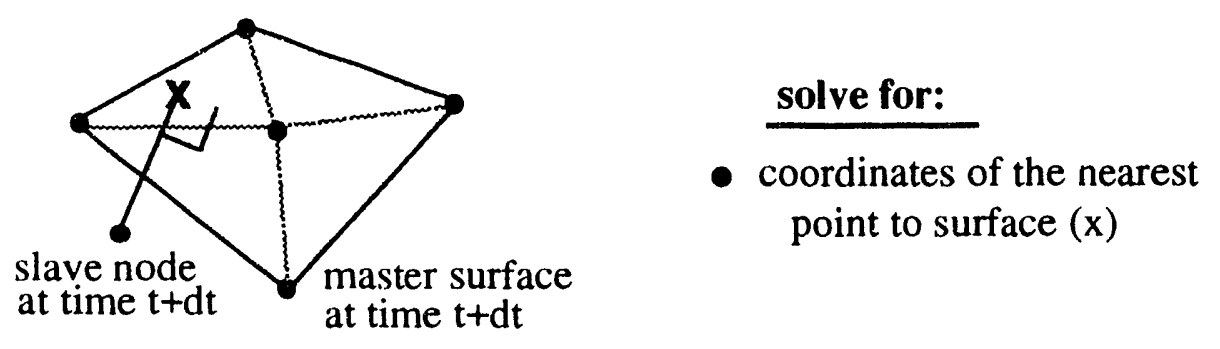

(b) static based contact check based on position

Figure 14. Master slave tracking using velocity and static contact check

\subsubsection{Velocity Based Contact Check}

The velocity based contact check makes use of the current position of the surfaces and the estimated velocities in the following time step. The contact check is restricted to a moving triangular master surface and a moving slave node for 3D, as shown in Figure 14a. 
The bi-linear quadrilateral master surface is subdivided into four triangular master surfaces. The four triangles are defined by the four corner nodes of the quadrilateral plus an added fifth node located at its centroid. In 2D, the master surface is a moving line, and is just a special case of the 3D moving contact presented below. A complete derivation of the velocity based contact check can be found in Appendix 1.

The equation of a triangular master surface (a plane) can be written as:

$$
a\left(x-x_{1}\right)+b\left(y-y_{1}\right)+c\left(z-z_{1}\right)=0
$$

The point $\left(x_{1}, y_{1}, z_{1}\right)$ is a node on the master surface, and $a, b$, and $c$ are components of the master surface normal $\overrightarrow{\mathrm{N}}_{\mathrm{m}}=\mathrm{a} \hat{\mathrm{i}}+\mathrm{b} \hat{\mathrm{j}}+\mathrm{c} \overrightarrow{\mathrm{k}}$, where:

$$
\begin{aligned}
& a=\left[\left(y_{1}-y_{3}\right)\left(z_{2}-z_{1}\right)-\left(y_{2}-y_{1}\right)\left(z_{1}-z_{3}\right)\right] \\
& b=\left[\left(x_{2}-x_{1}\right)\left(z_{1}-z_{3}\right)-\left(x_{1}-x_{3}\right)\left(z_{2}-z_{1}\right)\right] \\
& c=\left[\left(x_{1}-x_{3}\right)\left(y_{2}-y_{1}\right)-\left(x_{2}-x_{1}\right)\left(y_{1}-y_{3}\right)\right]
\end{aligned}
$$

Here, $x_{2}, y_{2}, z_{2}$ and $x_{3}, y_{3}, z_{3}$ are coordinates of the other nodes defining the triangular master surface. Now, note that the triangular surface is moving, so that:

$$
\begin{aligned}
& {\left[\left(x_{i}(t+\Delta t), y_{i}(t+\Delta t), z_{i}(t+\Delta t)\right)=\right.} \\
& \left.\quad\left(x_{i}(t)+\dot{x}_{i} \Delta t, \quad y_{i}(t)+\dot{y}_{i} \Delta t, \quad z_{i}(t)+\dot{z}_{i} \Delta t\right)\right], i=1,3
\end{aligned}
$$

and the slave node is also moving, so that:

$$
\begin{aligned}
& \left(x_{s}(t+\Delta t), y_{s}(t+\Delta t), z_{s}(t+\Delta t)\right)= \\
& \left(x_{S}(t)+\dot{x}_{s} \Delta t, \quad y_{s}(t)+\dot{y}_{s} \Delta t, \quad z_{s}(t)+\dot{z}_{s} \Delta t\right)
\end{aligned}
$$

Following these definitions, a time $\Delta \mathrm{t}$ is sought such that the slave node will be co-planar with the three nodes defining the triangular master surface. Such a time can be found (if it exists) by substituting equations (5), (6), and (7) into equation (4) and solving a cubic equation in $\Delta \mathrm{t}$ (see Appendix 1).

Suppose a solution $\Delta t=\Delta t_{c}$ is found. Then it also must satisfy two conditions for it to be considered a contact. The first is that the time $\Delta \mathrm{t}_{\mathrm{c}}$ occurs in the next time step increment, $0 \leq \Delta \mathrm{t}_{\mathrm{c}} \leq \mathrm{dt}$. The other condition is that the contact point:

$$
\left(x_{c}, y_{c}, z_{c}\right)=\left(x_{s}+\dot{x}_{s} \Delta t_{c}, y_{s}+\dot{y}_{s} \Delta t_{c}, z_{s}+\dot{z}_{s} \Delta t_{c}\right)
$$


must lie inside the edges of the quadrilateral master surface. This can be determined exactly by computing the local $\xi, \eta$ coordinates of the contact point on the quadrilateral master surface. Figure 15 shows how an initial estimate $\xi_{0}, \eta_{0}$ of the local coordinates can be computed. Four triangles are constructed by connecting the contact point with the four corners of the quadrilateral master surface. The contact point is inside the master surface when all four areas $A_{1}, A_{2}, A_{3}$, and $A_{4}$ are greater than or equal to zero and $A_{1}+A_{3}>0$ and $A_{2}+A_{4}>0$. If this condition is met, then an initial estimate of the local coordinates of the contact point can be found, as shown by the equations in Figure 15.

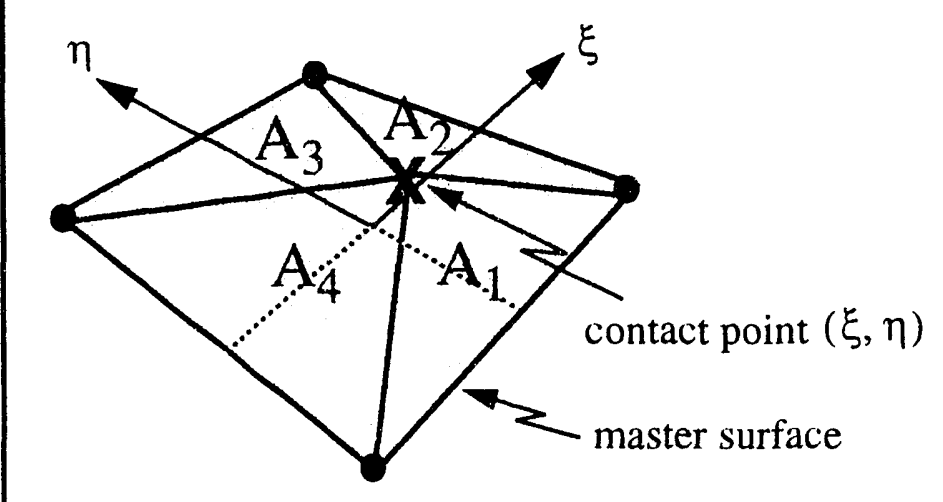

$$
\left.\begin{array}{c}
\begin{array}{c}
\text { initial estimate of } \\
\text { contact point }
\end{array} \\
\xi_{0}=2 \frac{A_{4}}{A_{2}+A_{4}}-1 \\
\eta_{0}=2 \frac{A_{1}}{A_{1}+A_{3}}-1
\end{array}\right)
$$

Figure 15. Initial estimates for the local coordinates of a contact point

The logic for these equations is based simply on the observation that $(\xi, \eta)=\left(\xi_{0}, \eta_{0}\right)$ is computed exactly for any rectangular quadrilateral and that the limiting values of $\xi$ and $\eta= \pm 1$ are computed exactly for any shaped quadrilateral. If the quadrilateral surface is a rectangle, then the proof is simple. If it is not, then by observation $\xi$ is computed exactly only when one of the areas $A_{2}$ or $A_{4}$ is zero. Likewise $\eta$ is computed exactly only when one of the areas $A_{1}$ or $A_{3}$ is zero. For example, suppose $A_{2}$ is zero then the estimated coordinate $\xi=\xi_{0}=1$ is exact. If $A_{4}$ is zero then the estimated coordinate $\xi=\xi_{0}=-1$ is also exact. If neither $\mathrm{A}_{2}$ or $\mathrm{A}_{4}$ are zero, then the equation gives a reasonable estimate of the contact point $\xi$. Improvements in the accuracy of the local coordinates can be achieved by performing Newton iterations on the nonlinear equations relating $\xi, \eta$, and $\zeta$ to $x_{c}, y_{c}$, and $z_{c}$ (see Appendix 1).

In certain cases there may be multiple master surfaces where contact is possible. The example problem shown in Figure 10 illustrates such a case in which a slave node contacts multiple master surfaces. For example, the slave node 27 could contact any one of the three master surfaces connected to node 18 . In these cases, a strength of contact check is used to determine the most opposed master surface where $\vec{N}_{m} \bullet\left(\vec{V}_{s}-\vec{V}_{m}\right)$ is minimized. In the example problem, master surface 5 is the most opposed master surface. 


\subsubsection{Static Contact Check}

The static contact check is used for those slave nodes already in contact with a master surface. The contact check is also restricted to a slave node contacting a triangular master surface. The calculations are based on the predicted configuration of the surface if the contact forces were removed from the surface nodes. This predicted configuration would obviously have slave nodes penetrating master surface elements. In order to bring these interpenetrating surfaces back into compliance, the slave node must be pushed back to the master surface. In determining the push back direction, a distinction is made between concave and convex surfaces.

The push back direction for a concave surface is determined simply by the minimum distance to the master surface as shown in Figure 16. The push back direction $\hat{\mathrm{p}}_{\mathrm{t}+\mathrm{dt}}$ can be along the master surface normal as shown in Figure 16a or it may be defined by the minimum distance to a vertex as shown in Figure 16b. This choice in pushback direction is done simply to detect an undetermined contact.

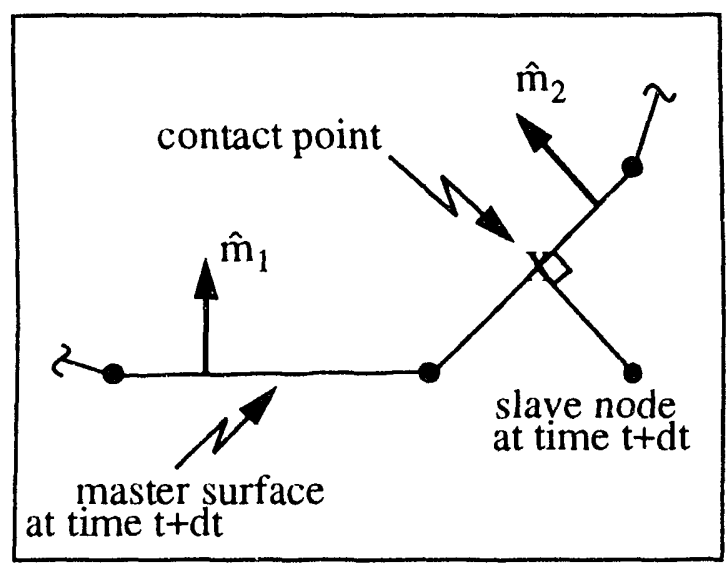

(a) closest point is on master surface 2

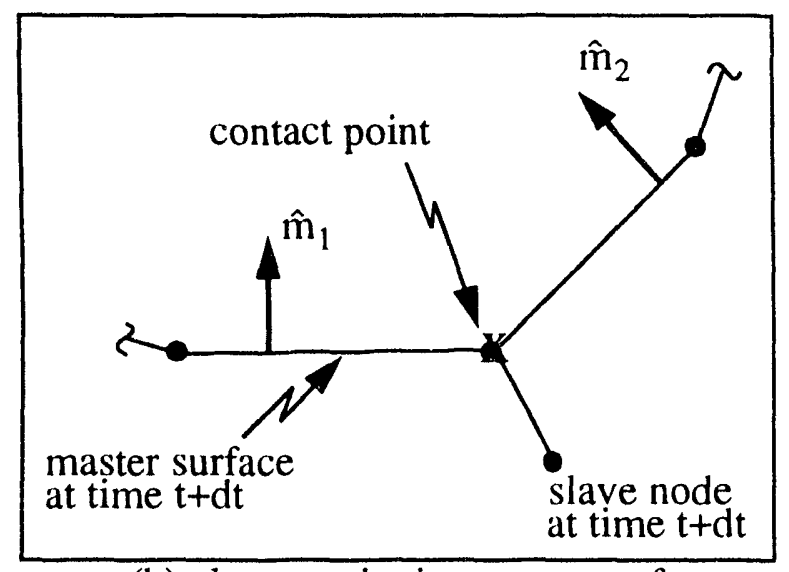

(b) closest point is on vertex of master surface 1 and 2

Figure 16. Push back direction for a concave surface based on minimum distance to master surface.

For a convex surface, the push back direction $\hat{\mathrm{p}}_{\mathrm{t}+\mathrm{dt}}$ is always along the normal of the master surface that the slave node was previously in contact with, i.e $\hat{m}_{p}$ in Figure 17. In Figure 17a the slave node was previously in contact with master surface $1\left(\hat{m}_{p}=\hat{m}_{1}\right)$ so the pushback direction is $\hat{\mathrm{p}}_{\mathrm{t}+\mathrm{dt}}=\hat{\mathrm{m}}_{\mathrm{p}}=\hat{\mathrm{m}}_{1}$. Again, in Figure $17 \mathrm{~b}$ the slave node was originally in contact with master surface 1 so $\hat{p}_{t+d t}=\hat{m}_{p}=\hat{m}_{1}$. After the slave node is pushed back to master surface 2 , the pushback direction is updated to reflect that $\hat{\mathrm{p}}_{\mathrm{t}+2 \mathrm{dt}}=\hat{\mathrm{m}}_{2}$. This avoids adding artificial slave node velocity due to a change in pushback direction, as illustrated in Figure 5. 


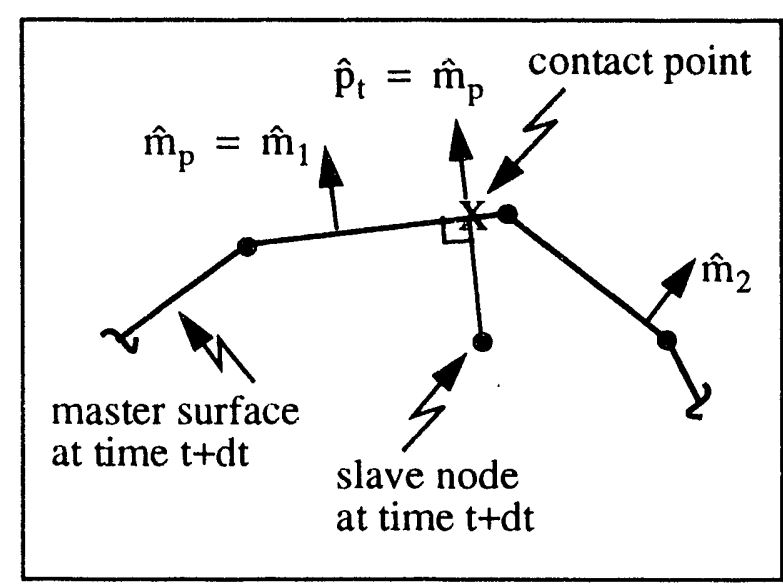

(a) closest point is on previous master surface

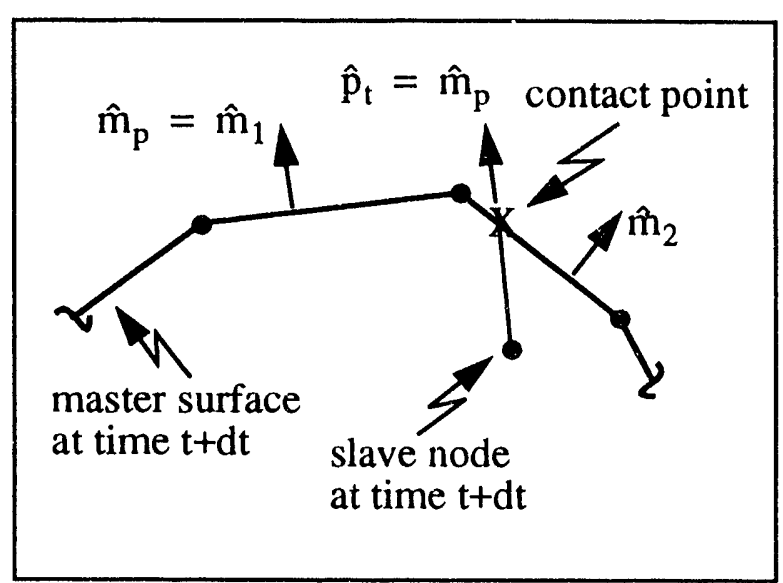

(b) closest point is neighboring master surface

Figure 17. Push back direction for convex surface based on previous master surface normal.

The contact point, $X$, on a convex surface is found by the intersection of the triangular master surface plane, Equation (4), and a line defined by the parametric equations:

$$
\begin{aligned}
& x=x_{s}+s\left(\hat{m}_{p} \bullet \dot{i}\right) \\
& y=y_{s}+s\left(\hat{m}_{p} \bullet \hat{j}\right) \\
& z=z_{s}+s\left(\hat{m}_{p} \bullet \vec{k}\right)
\end{aligned}
$$

Providing that the master surface and the line are not parallel, the parameter $s$ can be found as:

$$
s=\frac{a\left(x_{1}-x_{s}\right)+b\left(y_{1}-y_{s}\right)+c\left(z_{1}-z_{s}\right)}{a\left(\hat{m}_{p} \bullet \dot{i}\right)+b\left(\hat{m}_{p} \bullet \hat{j}\right)+c\left(\hat{m}_{p} \bullet \vec{k}\right)}
$$

Just as in the case of a velocity based contact check, there may be some instances where contact with multiple master surfaces is possible according to the static contact check. Again a strength of contact check is used to determine the master surface where $\vec{N}_{m} \bullet \vec{N}_{s}$ is minimized.

\subsection{Summary of Proposed Contact Detection Algorithm}

The proposed contact detection algorithm outlined in this section offers some advantages over the currently used algorithm in the Sandia codes. It does so by separately considering a fast, memory efficient, global search for potential contacts, and a much more accurate detailed contact check: 
- The efficient global search allows for global contact. This means that added capability for modelling self contacting structures and eroding or tearing surfaces is now available. Another benefit of the efficient global contact search is that fewer slave node - master surface pairs are found as potential contacts. This is possible by taking advantage of both the known location of the contacting surfaces and their velocities in constructing a master surface capture box. The capture box ensures that a minimum number of slave nodes are paired with the master surface.

- The detailed contact check is more accurate in determining the point of contact, amount of penetration, and the direction of pushback. This results in a physically correct determination of contact constraints. The improved accuracy has also reduced the number of iterations required for convergence in the iterative solvers in the Sandia quasistatic codes. 


\section{Surface Definition Algorithm}

The proposed contact detection algorithm described in the previous section discussed the details of the neighborhood identification and global search, and the detailed contact check. Implied in the discussion was the definition of the entities involved in contact, namely the slave nodes and the master surfaces. Typically this is done using preprocessors such as FASTQ [21], GJOIN [22], GEN3D [23], and GENSHELL [24] to define meshes and side sets. Side sets are a collection of element faces and their corresponding nodal points. The contact algorithm would then require as input the listing of these side sets (without requiring side set pairing). In many applications, such as self contact, this kind of input is all that is required. For other applications such as tearing or eroding surfaces, the contact surface is changing during the execution of the problem. In these types of problems, a dynamically defined surface (composed of all master surfaces and slave nodes on the surface) is essential.

For this purpose, an algorithm for automatically determining the surface of an arbitrary mesh composed of hexahedral and shell elements is proposed. The surface definition algorithm uses a data structure that allows the initial surface definition and an incrementally updated surface when necessary. In the algorithm, shell elements are considered as a subset of hexahedral elements. For clarity, the following discussion is limited to a mesh composed of hexahedral elements. The algorithm requires $6 \times$ ne memory locations for a mesh composed of ne 8 -node hexahedral elements and involves two simple steps. The first step is to construct a face id for every element face:

$$
\text { faceid }=n_{\text {diag }}+n_{\min } \times \text { nnod }
$$

where nnod is the total number of nodes in the problem, $n_{\min }$ is the smallest global node number defining the element face, and $n_{\text {diag }}$ is the global node number that is diagonal to the smallest global node $n_{\min }$. Step two is to search for all face id's for any non-repeated numbers which will correspond to the faces that are on the surface. The search is efficiently done using the CRAY UNICOS library routine wheneq [25]. Figure 18 shows an example of a 3D mesh composed of two hexahedral elements.

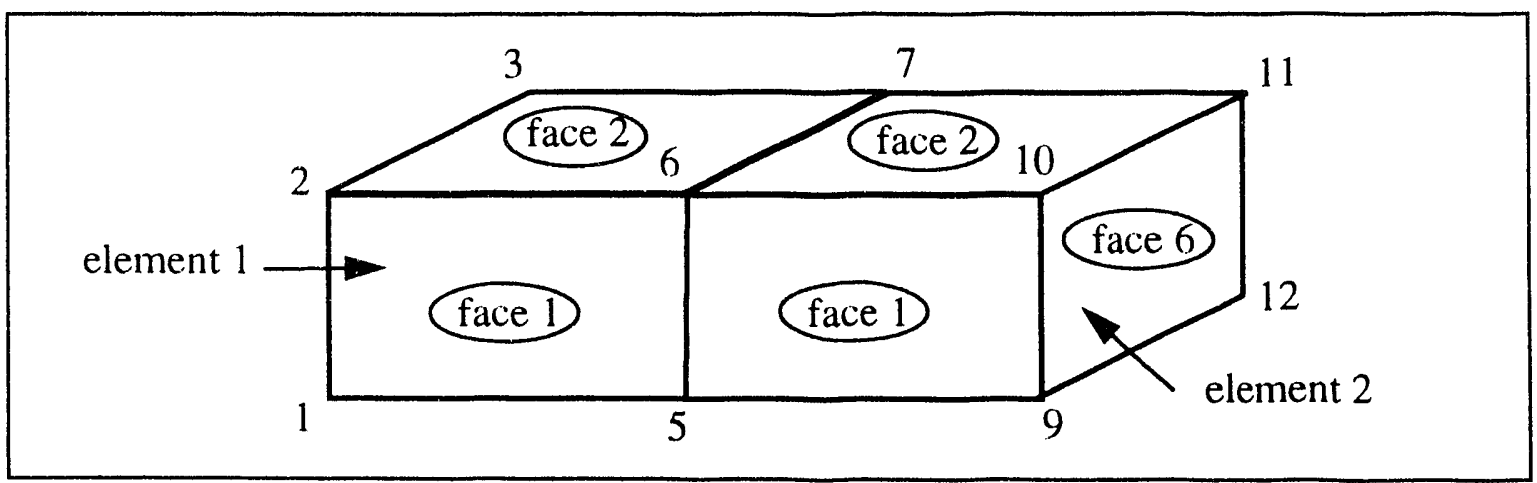

Figure 18. Example mesh for surface definition algorithm 
Table 1 lists the 12 element faces in the mesh, their connectivity, $n_{\text {min }}, n_{\text {diag }}$, and their face id. In the example problem, all element faces are on the surface except element 1 - face 6 and element 2 - face 5 (which have a the same face id of 67).

\begin{tabular}{||l|l|l|l|l|l||}
\hline \multicolumn{6}{||}{ Table 1. Surface definition algorithm example } \\
\hline $\begin{array}{l}\text { element } \\
\text { number } \\
\text { (iele) }\end{array}$ & $\begin{array}{l}\text { face } \\
\text { number } \\
\text { (iface) }\end{array}$ & connectivity & $n_{\min }$ & $n_{\text {diag }}$ & $\begin{array}{l}\text { smap } \\
\text { (face id) }\end{array}$ \\
\hline 1 & 1 & 1562 & 1 & 6 & 18 \\
\hline 1 & 2 & 2673 & 2 & 7 & 31 \\
\hline 1 & 3 & 3784 & 3 & 8 & 44 \\
\hline 1 & 4 & 4851 & 1 & 8 & 20 \\
\hline 1 & 5 & 1234 & 1 & 3 & 15 \\
\hline 1 & 6 & 5876 & 5 & 7 & 67 \\
\hline 2 & 1 & 59106 & 5 & 10 & 70 \\
\hline 2 & 2 & 610117 & 6 & 11 & 83 \\
\hline 2 & 3 & 711128 & 7 & 12 & 96 \\
\hline 2 & 4 & 81295 & 5 & 12 & 72 \\
\hline 2 & 5 & 5678 & 5 & 7 & 67 \\
\hline 2 & 6 & 9121110 & 9 & 11 & 119 \\
\hline
\end{tabular}

The result of collecting all the element faces with unique face ids is a list of the master surfaces. The slave nodes can be determined by looping through the master surface list and flagging the nodal points defined by the master surface connectivity.

The array called smap initially stores the faceid of each face, as shown in Table 1. For every element face on the surface (ones with a unique face id), a zero is over-written in $\operatorname{smap}\left(6^{*}(\right.$ iele-1)+iface). For those interior element faces (ones without unique face ids), a pointer to the opposing face is stored in the array, smap:

$$
\begin{aligned}
& \operatorname{smap}\left(6\left(\text { iele }_{i}-1\right)+\text { iface }_{i}\right)=6\left(\text { iele }_{j}-1\right)+\text { iface }_{j} \\
& \operatorname{smap}_{\left(6\left(\text { iele }_{j}-1\right)+\text { iface }_{j}\right)}=6\left(\text { iele }_{i}-1\right)+\text { iface }_{i}
\end{aligned}
$$

Using this surface map array, the surface can be incrementally updated as elements are deleted. For the current example there are only two opposing faces, element 1 face 6 and element 2 face 5 , so that $\operatorname{smap}(6)=11, \operatorname{smap}(11)=6$, and all other positions in smap would be zero.

This idea of collecting master surfaces and slave nodes into a heap allows modelling of contact between a variety of finite element types. For example, the nodal points of elements such as beams and trusses can be added to the slave node list. Also, the potential contacts in a problem coupling the finite element method with other methods can be modelled. For example, particle methods such as Smooth Particle Hydrodynamics (SPH) [26] or Particle-InCell (PIC) [27] can be easily coupled by adding the particles to the slave node list. 


\section{Example Problems}

The examples considered in this section demonstrate both the improvements in the accuracy of contact detection and the added capabilities that are possible with the global search algorithm. The improvements in the accuracy of contact detection are demonstrated with an example of two elastic blocks contacting and an example of contact induced chatter or ringing. Some of the added capabilities include modelling (1) structures that buckle and fold unto themselves, (2) structures that have materials which tear and create new surfaces, (3) problems where multiple body contact/inspact is occurring, and (4) problems where considerable sliding between bodies is occurring. (The necessary files for constructing the input for all example problems are in Appendix 2)

\subsection{Contact of Elastic Blocks}

The example of two blocks impacting one another is a simple demonstration of the need for an accurate detailed contact check. The new contact algorithm correctly chooses the most opposed master surface for each slave node in the two cases shown in Figures 19 and 20. The two different cases are described as follows:

Case 1. The elastic blocks already discussed in the previous sections and shown in Figure 10 are impacting at a velocity $v_{y}=500 \mathrm{in} / \mathrm{s}$, as shown in Figure 19. Each corner node has the potential of contacting three different master surfaces, and each edge node has the potential of contacting four different master surfaces. The new contact algorithm duplicates the results of the old algorithm by correctly enforcing the most opposed contacts.

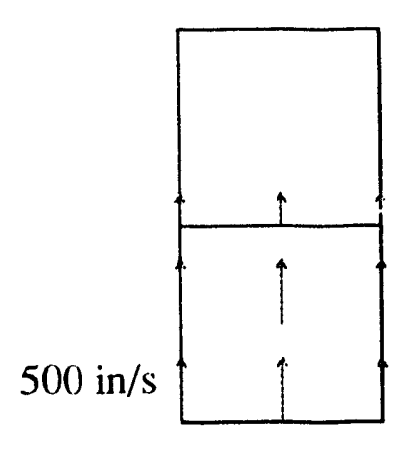

$$
\text { impact }(t=0)
$$

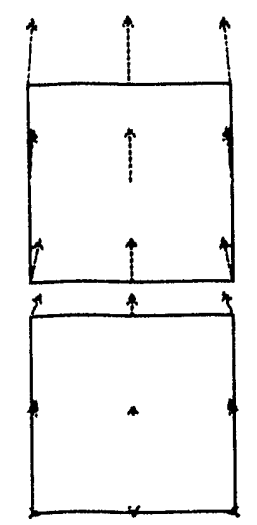

$\mathrm{t}=0.5$ milliseconds

Figure 19. End-on impact of two blocks 
Case 2. The same two blocks in Figure 10 are repositioned such that a corner of Block 1 is impacting a corner of Block 2, as shown in Figure 20. The old static contact check, which is based on surface normals, chooses the incorrect master surface causing both blocks to rotate clockwise. By incorrectly determining the pushback direction, a large increase in kinetic energy (from 92.5 in-lbs to 1300 in-lbs) results. The new contact detection algorithm correctly determines the initial contact using the velocity based check and the subsequent updated contact using the static based check. This results in the correct counterclockwise rotation of both hlocks.

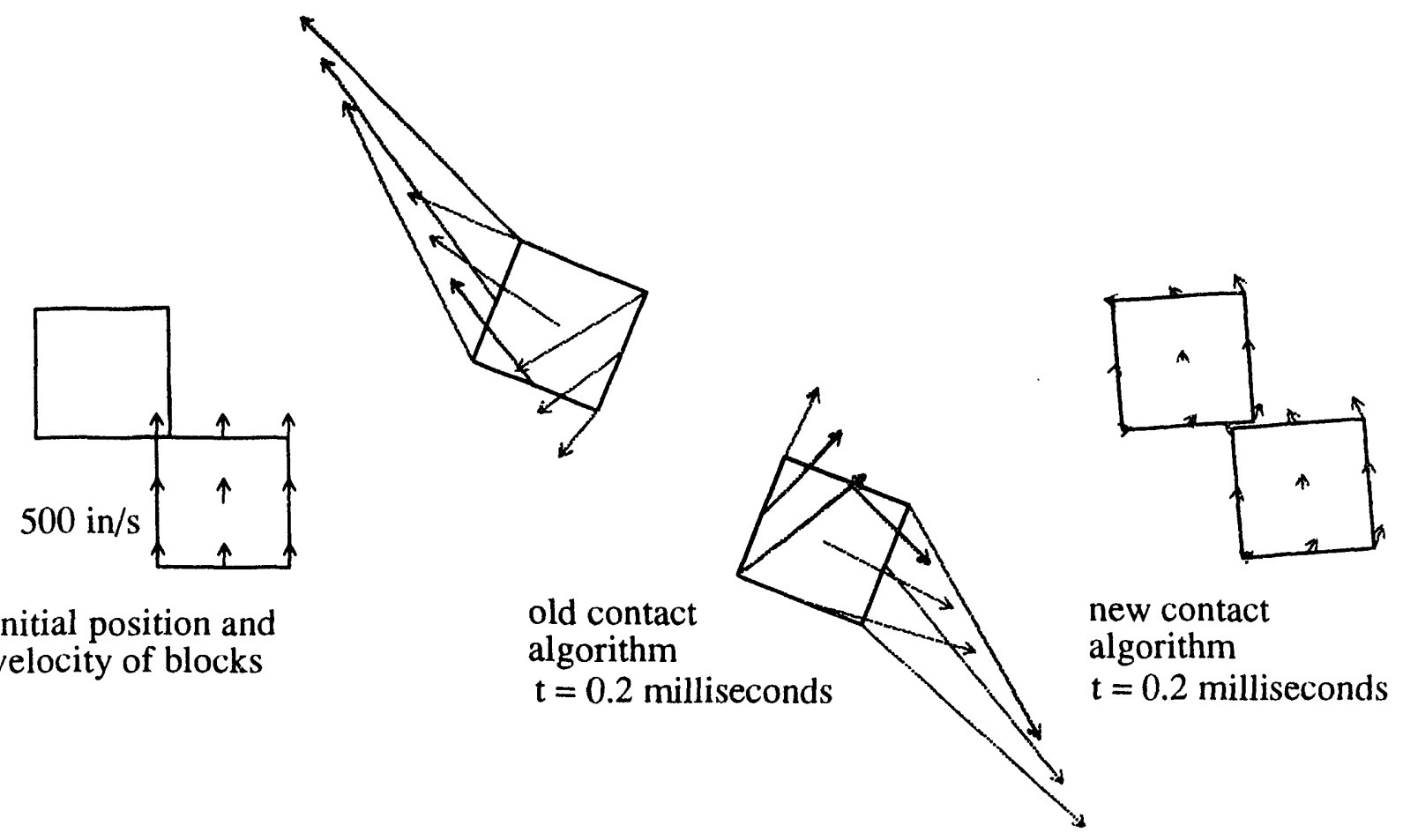

Figure 20. Corner impact of two blocks 


\subsection{Contact Chatter under High Normal Loads: Pressure Loading of Two Elastic Bodies}

One of the difficulties with two curved surfaces contacting each other under high normal loads is the accurate determination of the push back direction. The example shown in Figure 21 has a semicircular rod that is pushed into a semicircular cavity. A pressure load is applied to the flat surface of the rod. All the nodes on each contact surface are initially aligned with each other, so that as the pressure is ramped up, the slave nodes on the semicircle (convex surface) must be pushed back to the vertices of the master surfaces on the cavity (concave surface). The old algorithm incorrectly determines the pushback direction and introduces noise (contact chatter) into the solution. This eventually accumulates over many time steps and results in mesh hourglassing and increased kinetic energy, as shown in Figure 22. The new contact detection algorithm correctly determines the pushback dirf ction and does not introduce noise.

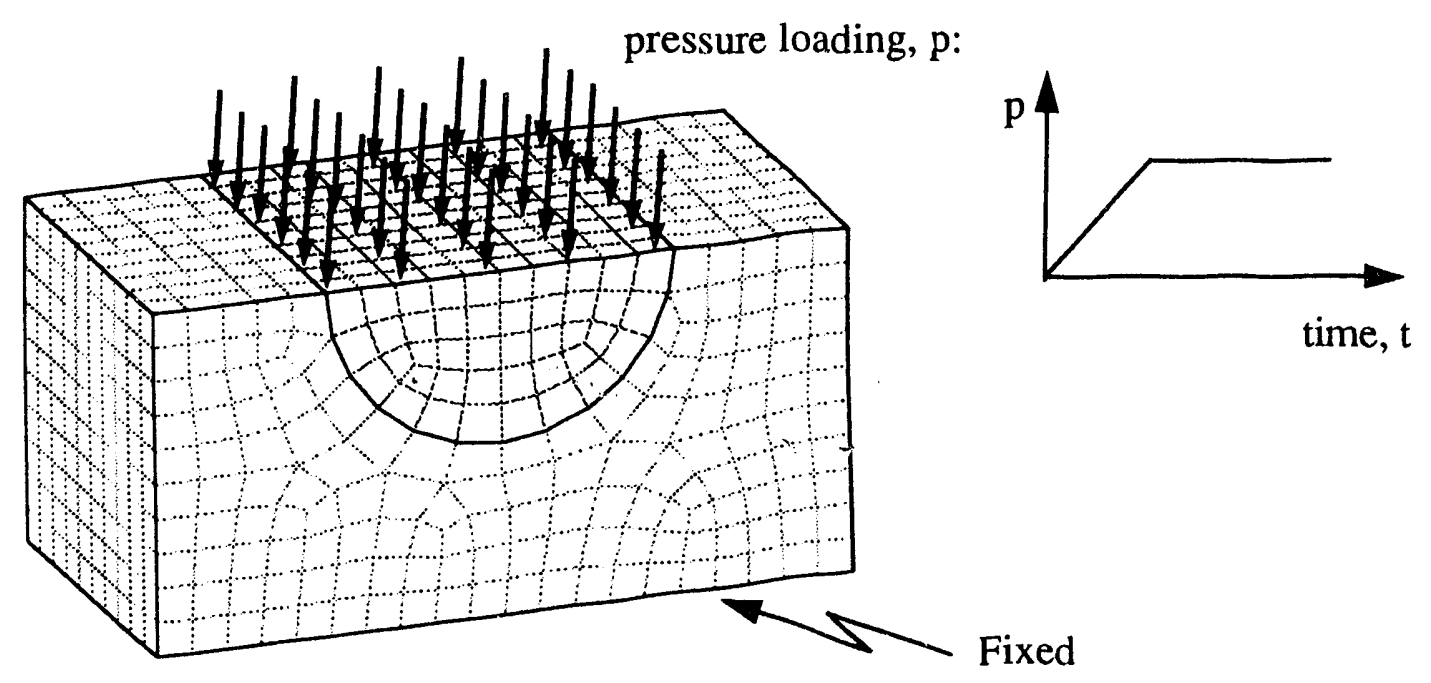

Figure 21. Two elastic bodies contacting under high normal load. 

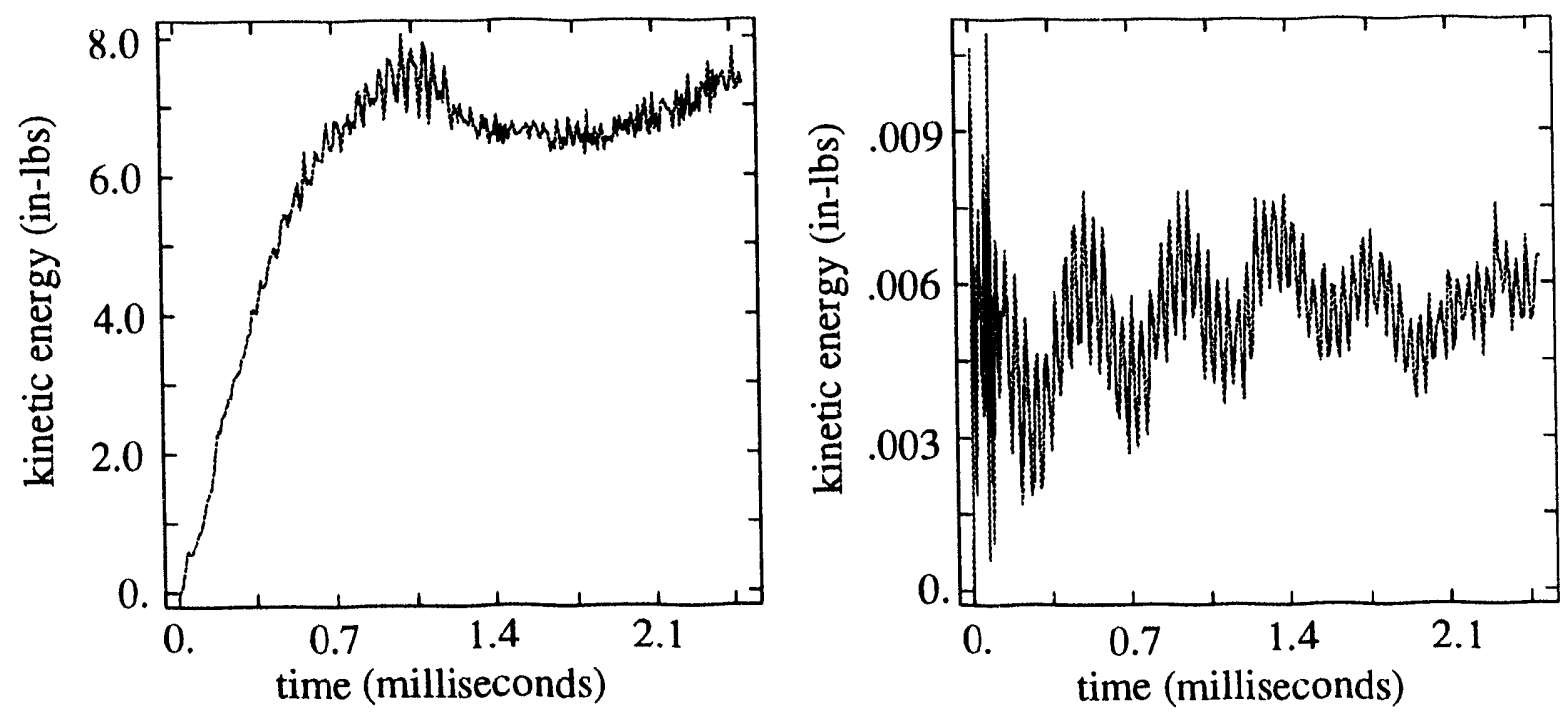

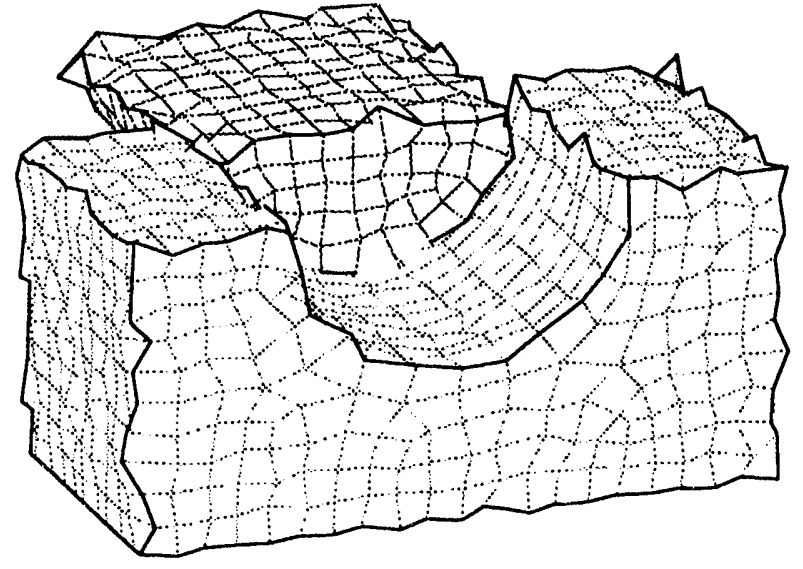

(a) old algorithm

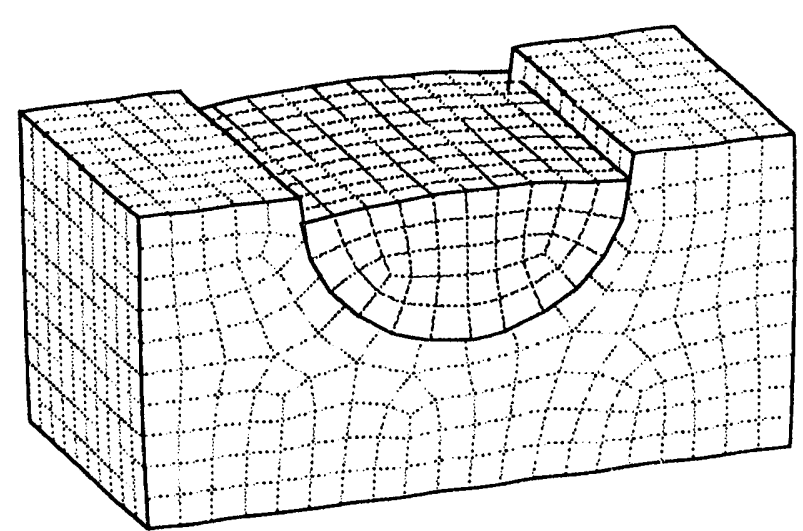

(b) new algorithm

Figure 22. Kinetic energy history and deformed shape (displacements magnified by $100 \mathrm{x}$ ) using old and new contact detection algorithm 


\subsection{Self-Contacting Impact: Buckling of Shell-Like Structures}

The folloving example demonstrates the self contacting capability of the contact detection algorithm. This feature is important for modelling crash dynamics where buckling, tearing, and self contact is common. The elastic-plastic shell-like (can) structure shown in Figure 23 is impacted by an elastic plastic plate. The can is 0.25 inches thick, has an inside radius of 5 inches, and is 15 inches long. The bottom of the can is constrained in all directions. The $22 \times 11$ in. plate is 2.5 inches thick and is initially tilted at a 10 degree angle as it impacts the can at $5000 \mathrm{in} / \mathrm{s}$.

The contact detection algorithm currently in PRONTO3D is a surface tracking algorithm that requires surface pairing. The amount of user intervention required to intermittently define and pair many contact surfaces would make this problem impractical to solve using the current algorithm. In contrast, the new algorithm requires two surface ids (one for the plate and one for the can). Contact between any of the slave nodes and master surfaces on both surface sets are detected during ine analysis. The deformed shapes at various times are shown in Figure 24. The self-contact of the buckled can is evident in several locations.

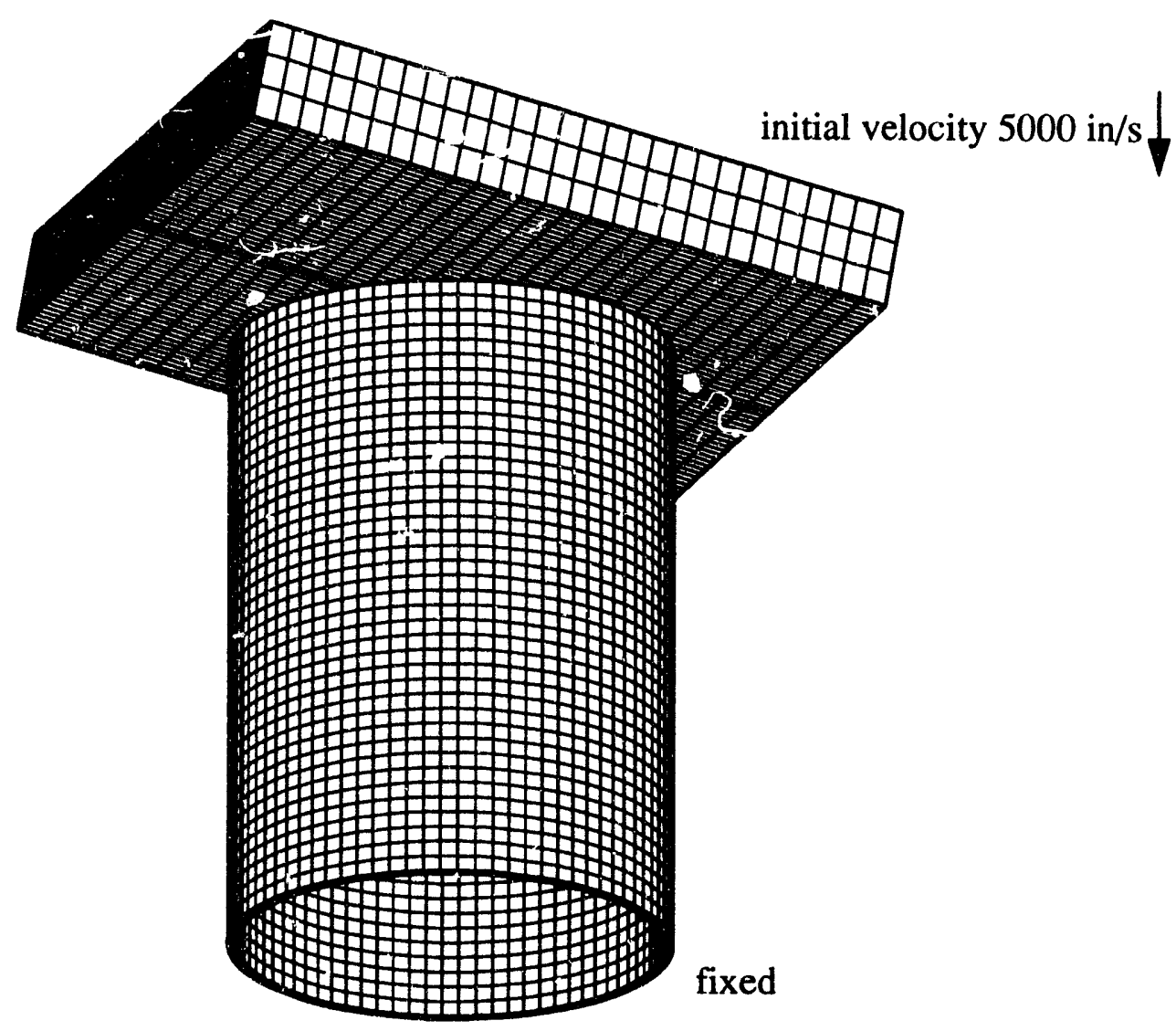

Figure 23. Finite element mesh of an elastic plate impacting an elastic-plastic can 


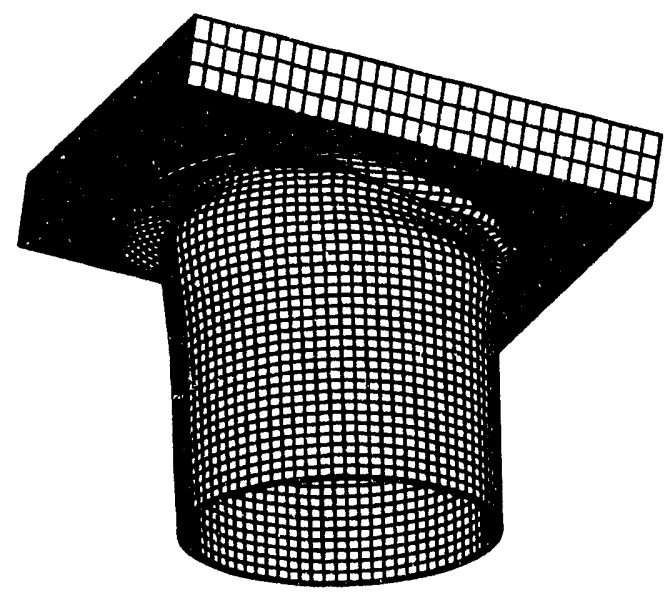

(a) $\mathrm{t}=1.1$ milliseconds

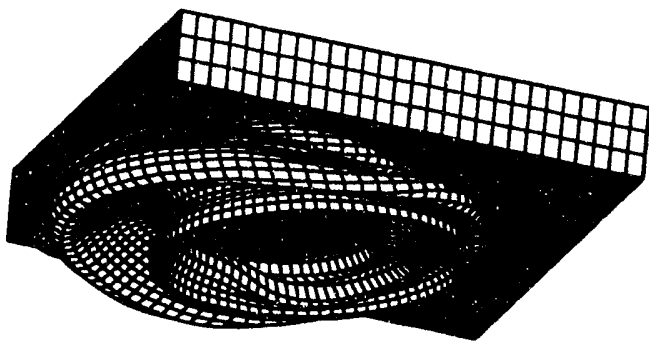

(c) $\mathrm{t}=3.3 \mathrm{~ms}$

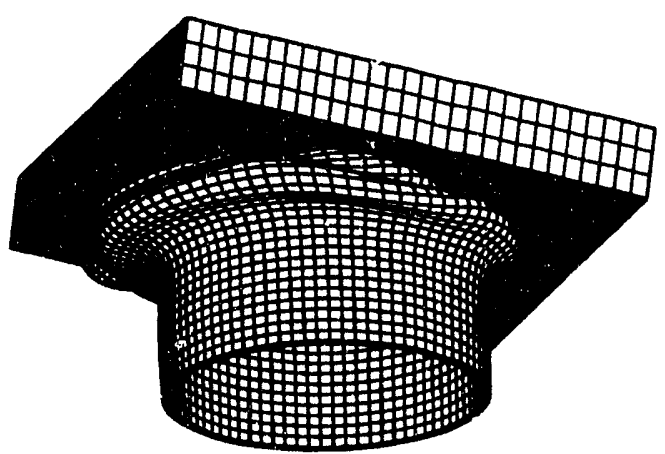

(b) $\mathrm{t}=2.2 \mathrm{~ms}$

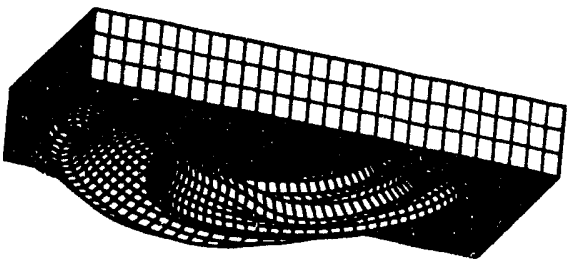

(d) half-section at t $=3.3 \mathrm{~ms}$

Figure 24. Deformed meshes of the buckled can at various times 


\subsection{Automatic Contact Surface Redefinition: Cutting of a Steel Pipe}

In the following example, the capability of automatically redefining the contact surface is exercised. For this problem, and others like it, the new surface that is generated as a result of tearing can find itself in contact with other surfaces of the body. The current capabilities of the Sandia codes do not include a periodic redefinition of the contact surface. The new algorithm automatically redefines the surface after any elements are deleted.

The algorithm is demonstrated with a simulation of a pipe cutting process. The process involves a hardened, 0.25 inch thick, wedged-shape cutting blade that is forced through a two inch, schedule 40 pipe that is resting on a support, as shown in Figure 25 . The blade initially indents and punctures through one side of the pipe and then progressively tears the pipe walls, as shown in Figure 26. The tear is simulated by deleting elements in which the material damage has accumulated to 0.9 . The power law hardening material accumulates damage when it exceeds a failure strain of 1.27 and is loaded under hydrostatic tension [28]. The newly created surface is automatically included in the contact algorithm by redefining the surface after any elements are deleted. As the simulation progresses, the edges of the tear are in contact with the moving cutting blade, as shown in Figure 26. The simulation continues until the blade cuts through the pipe at approximately 3 milliseconds.

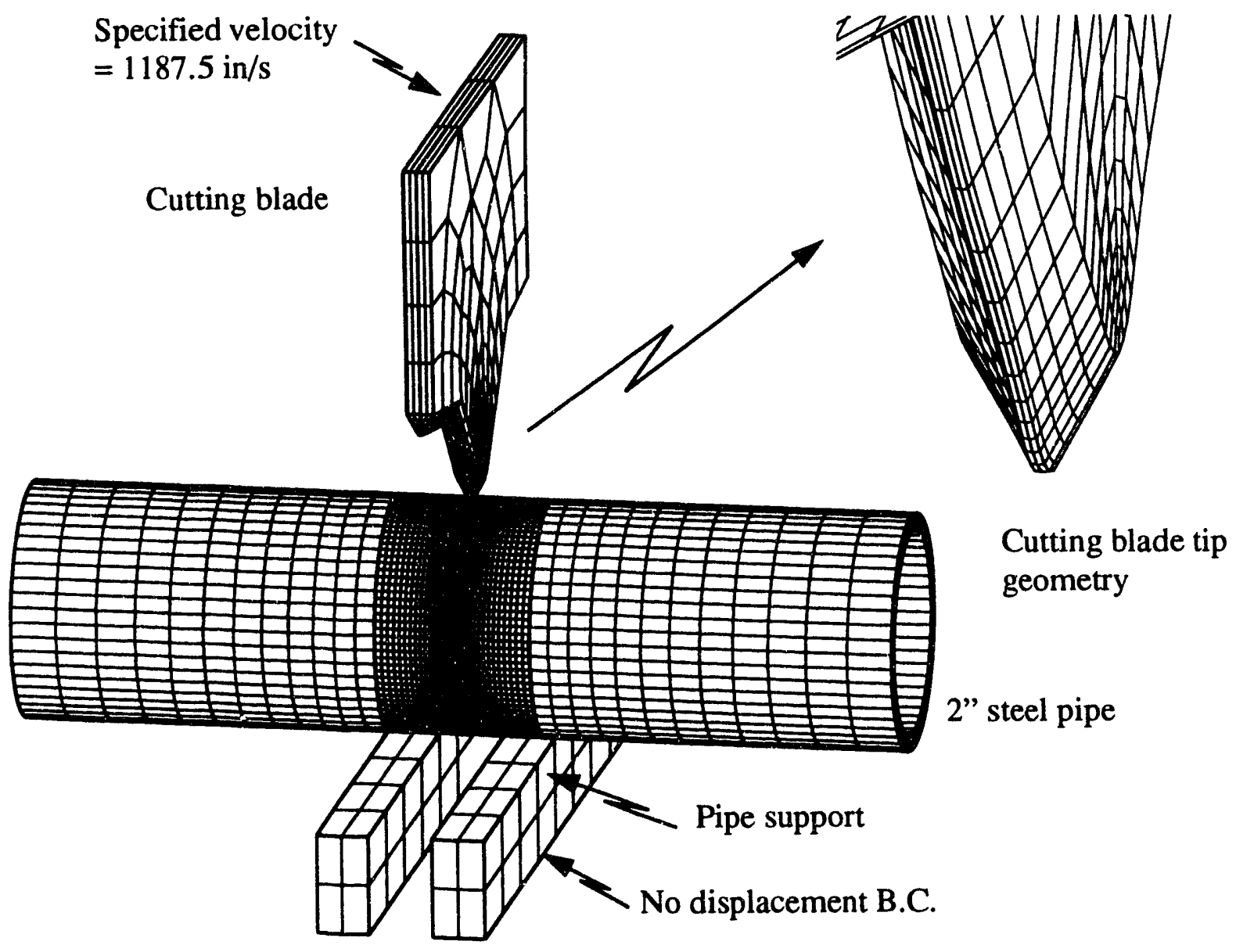

Figure 25. Finite element model for simulating the cutting of a 2 inch steel pipe. 

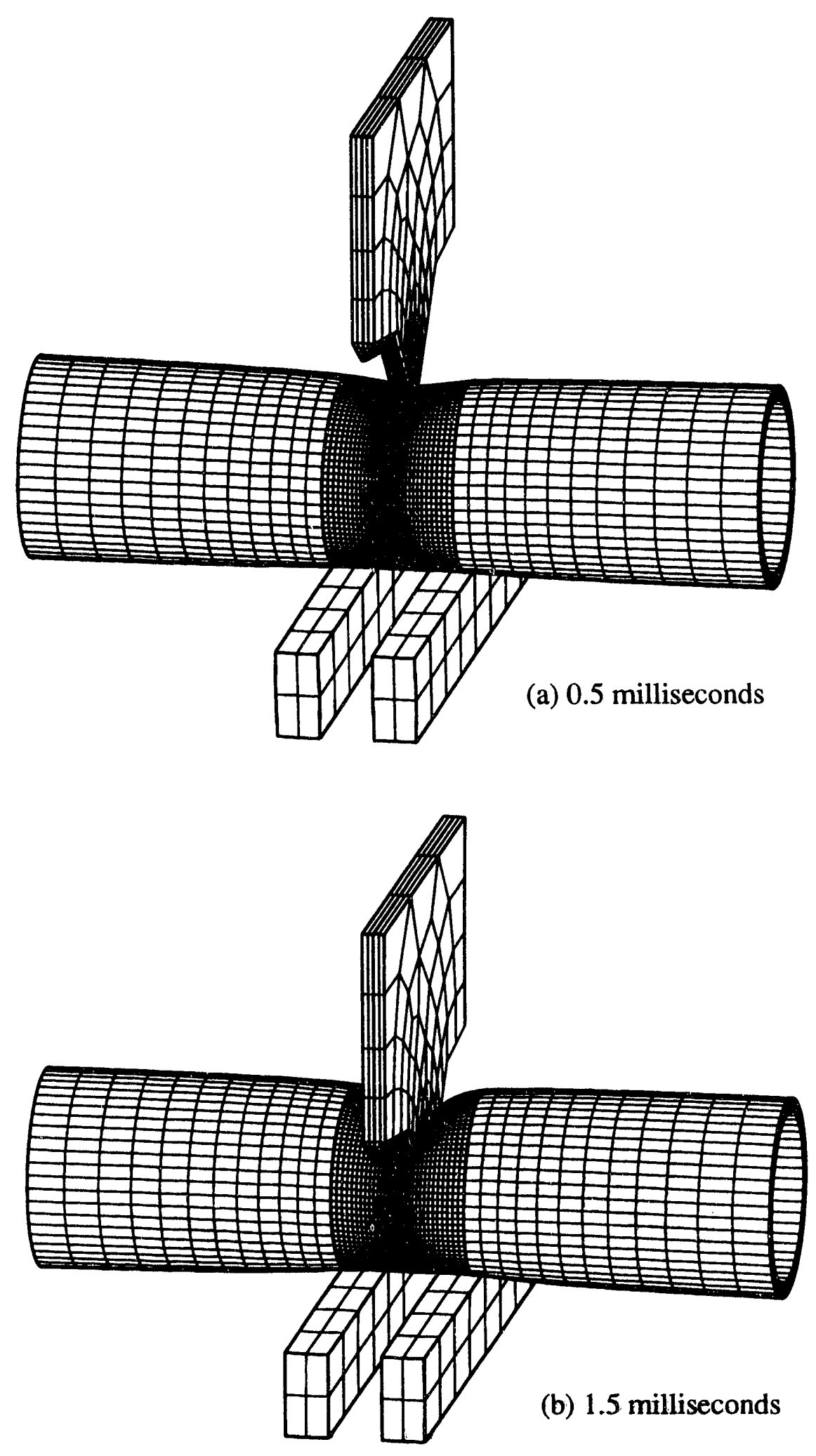

Figure 26. Pipe cutting simulation results at various times 

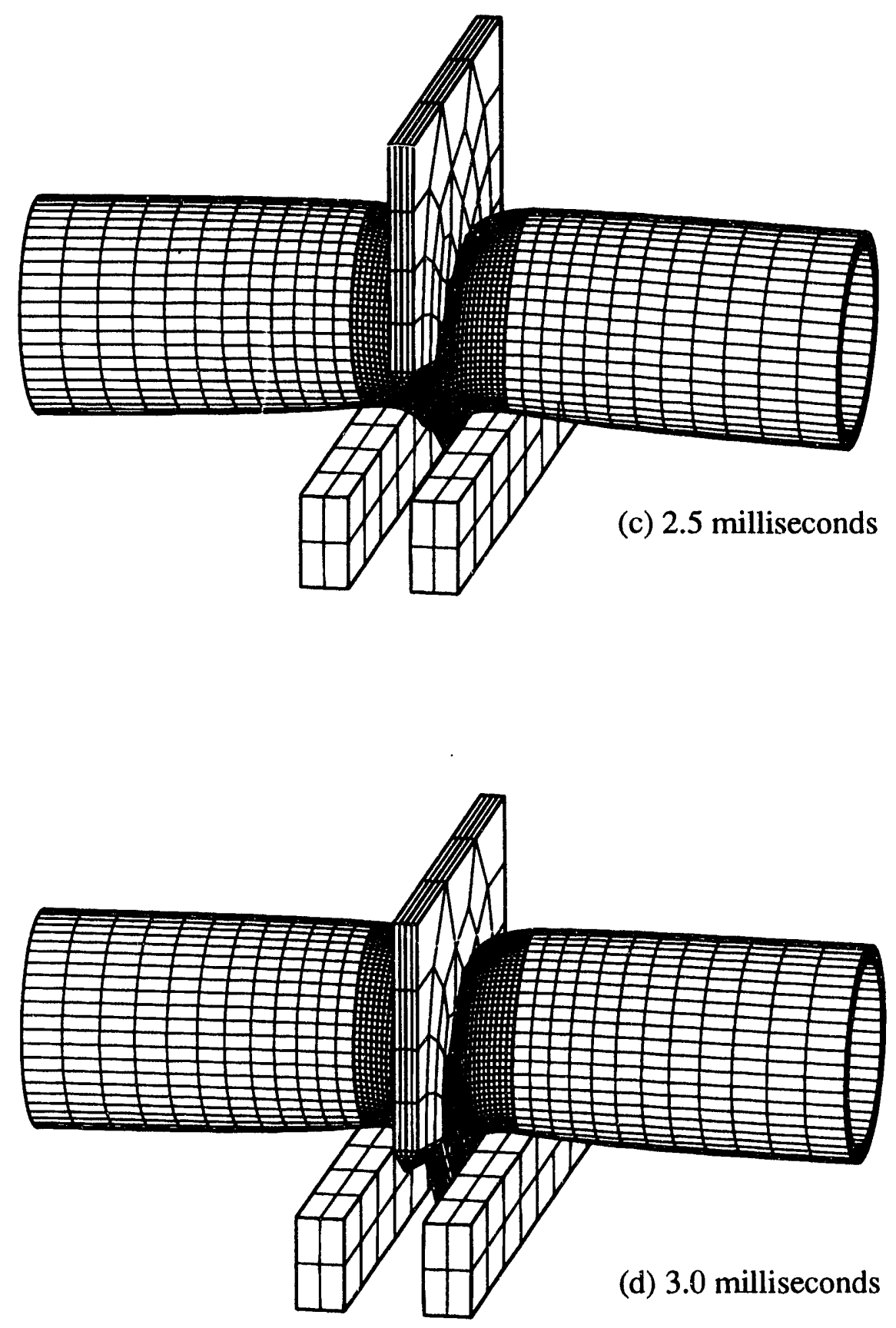

Figure 26 (cont'd). Pipe cutting simulation results at various times 


\subsection{Multi-Body Impact: Elastic-Plastic Bar impacting Bricks}

One of the added capabilities of the new contact detection algorithm is the efficient modelling of multi-body impact without a-priori definition of contact surfaces. The example, shown in Figure 27, has an elastic-plastic bar impacting a stack of 17 elastic bricks. A stationary elasticplastic wall is also resting against the stack of bricks. All slave nodes and master surfaces on the bodies were automatically defined using the algorithm described in Section 4.

For problems where random contact is anticipated, as in this example, each body could potentially impact any other body. For a contact-pairing algorithm, $19^{2}$ contact pairs would be necessary, with each pair having $2 n$ slave nodes. For the new global contact searching algorithm, one search with $19 \mathrm{n}$ slave nodes is necessary. Assuming that each block has $\mathrm{n} \approx 50$ slave nodes, $19^{2}$ pairs would require $19^{2}(2 n \log (2 n))=239,843$ comparisons, whereas the new global contact searching algorithm would require only $19 n \log _{2}(19 n)=9397$ comparisons.

Another important feature of the contact detection algorithm is the spatial independence of the nlogn vectorized sorting algorithm. The speed and efficiency of the algorithm is nearly independent of the location of the bricks. This becomes particularly important late in this example since the volume that the bodies occupy is increasing, as shown in Figure 28.

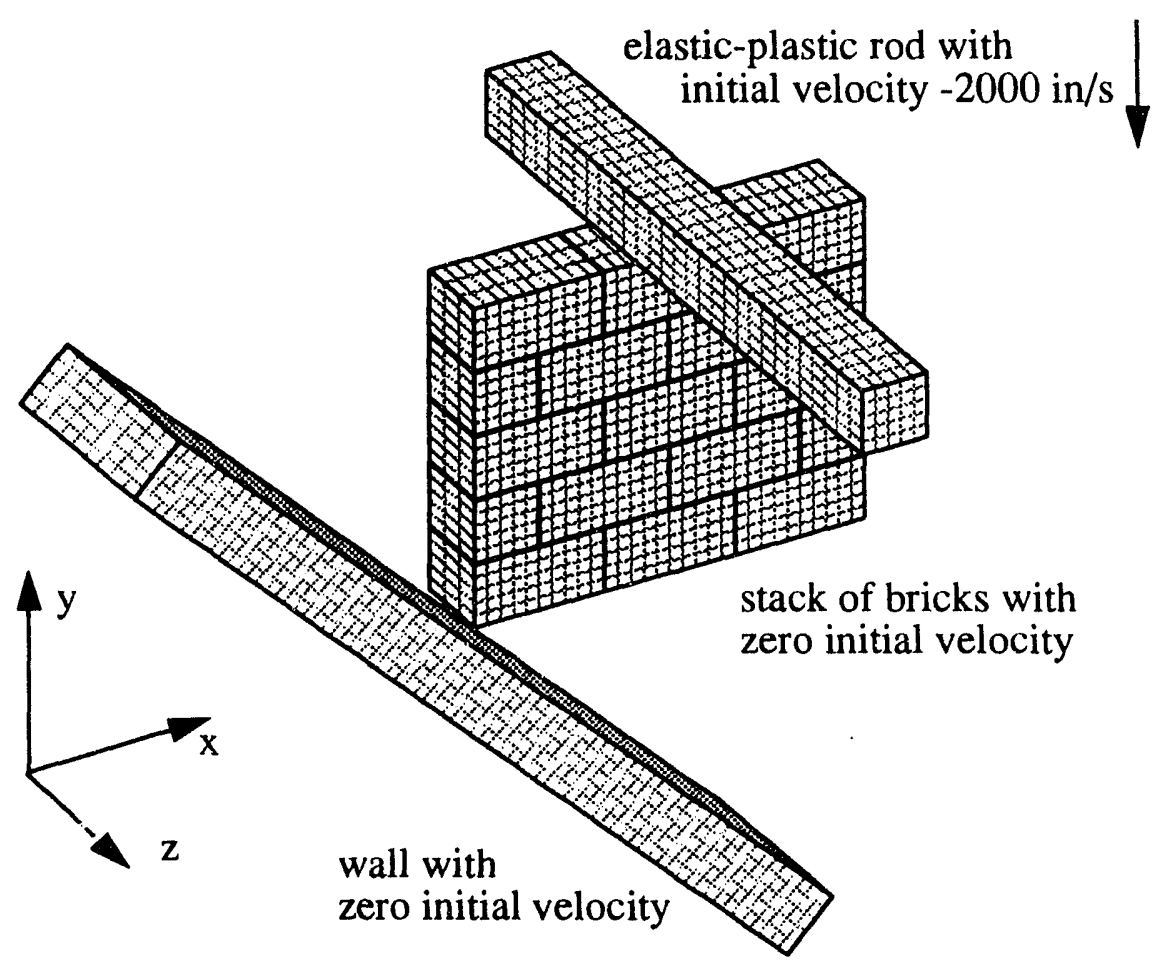

Figure 27. Elastic-plastic bar impacting a stack of 17 elastic bricks 

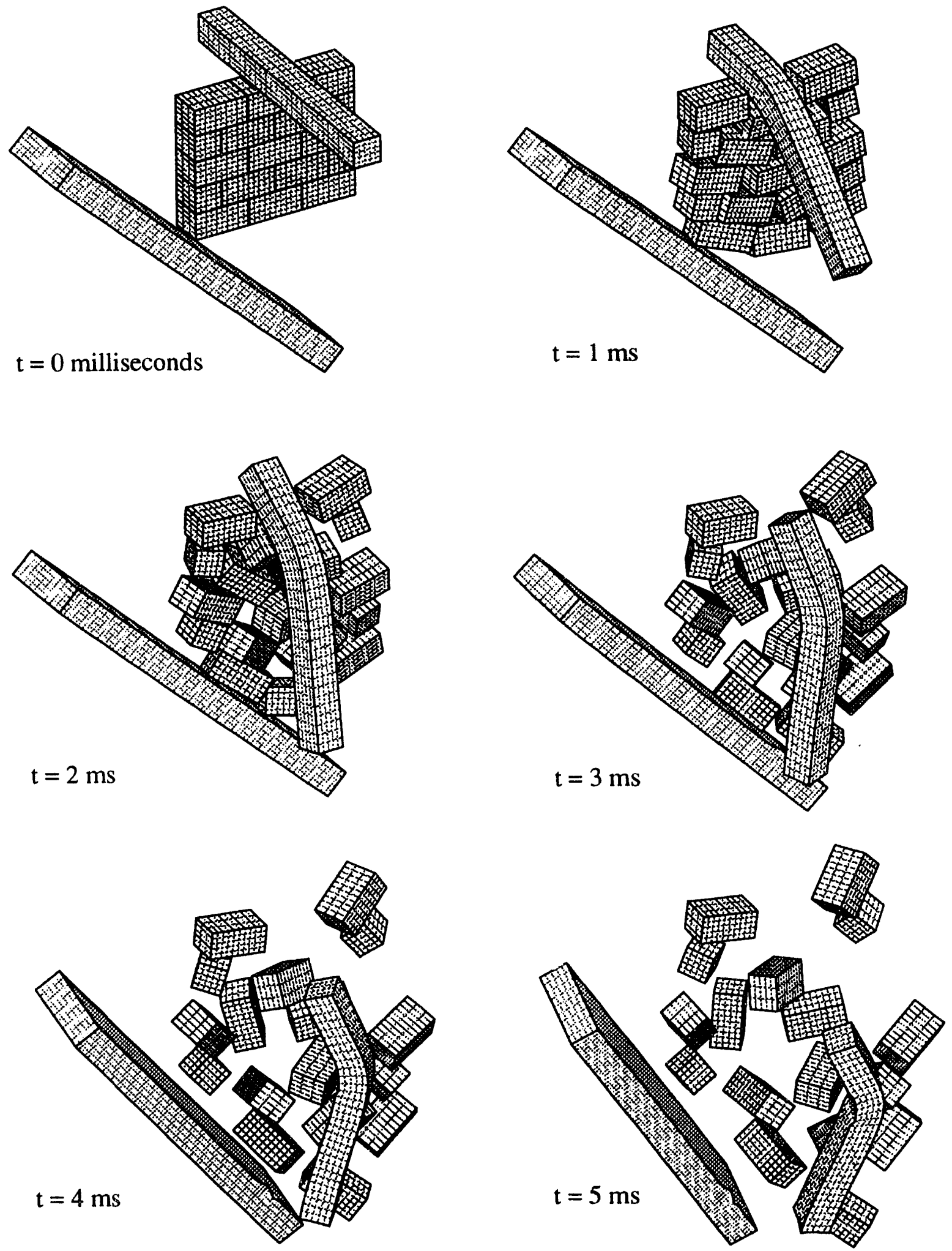

Figure 28. Multi-body impact simulation results at various times 


\subsection{Large Sliding Contact: Elastic-Plastic Forging of a Copper Billet}

The following example demonstrates the capability of modelling large sliding contact typical in large deformation forming and forging. The quasistatic axisymmetric forging of a copper billet shown in Figure 29 was simulated with the program SANTOS. The billet has an initial radius of 2 inches and height of 5 inches. Figure 30 shows a series of deformed meshes as the billet is forged. As the billet is compressed, it slides horizontally until the die cavity is filled. After further compression, the billet is forged around the die corner forming the rivet head.

There are two difficulties related to contact detection in this example. The first is the accurate determination of the pushback direction of the slave node in the lower corner of the die. It is initially constrained on the horizontal master surface as the billet is compressed. Then, just as the billet fills the die cavity, the slave node is constrained on the vertical master surface. Upon further loading, gradual adjustments in the pushback direction are automatically made so that it is constrained to the vertex of the horizontal and vertical master surface. (The current tracking algorithms do not push the slave node back to the vertex of the two master surfaces. This results in contact chatter similar to that described in example 5.2.)

Another difficulty arises from the large sliding contact of the material in the billet around the die corner (shown enlarged in Figure 30). The new contact detection algorithm determines the updated contact point and pushback direction such that the slave nodes on the billet are not given any added artificial velocity.

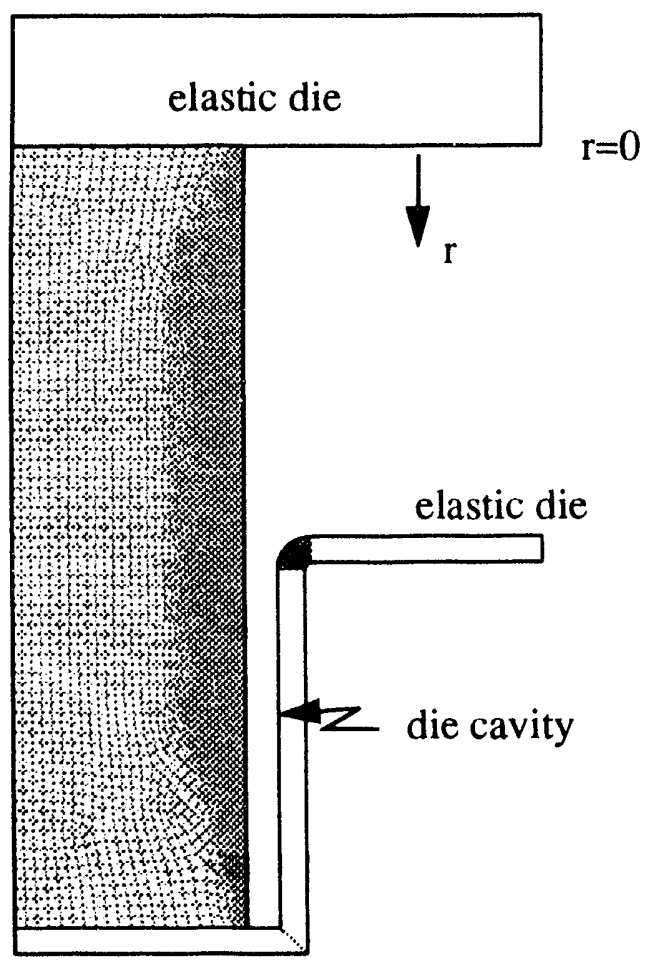

Figure 29. Finite element mesh of a rivet forging process 

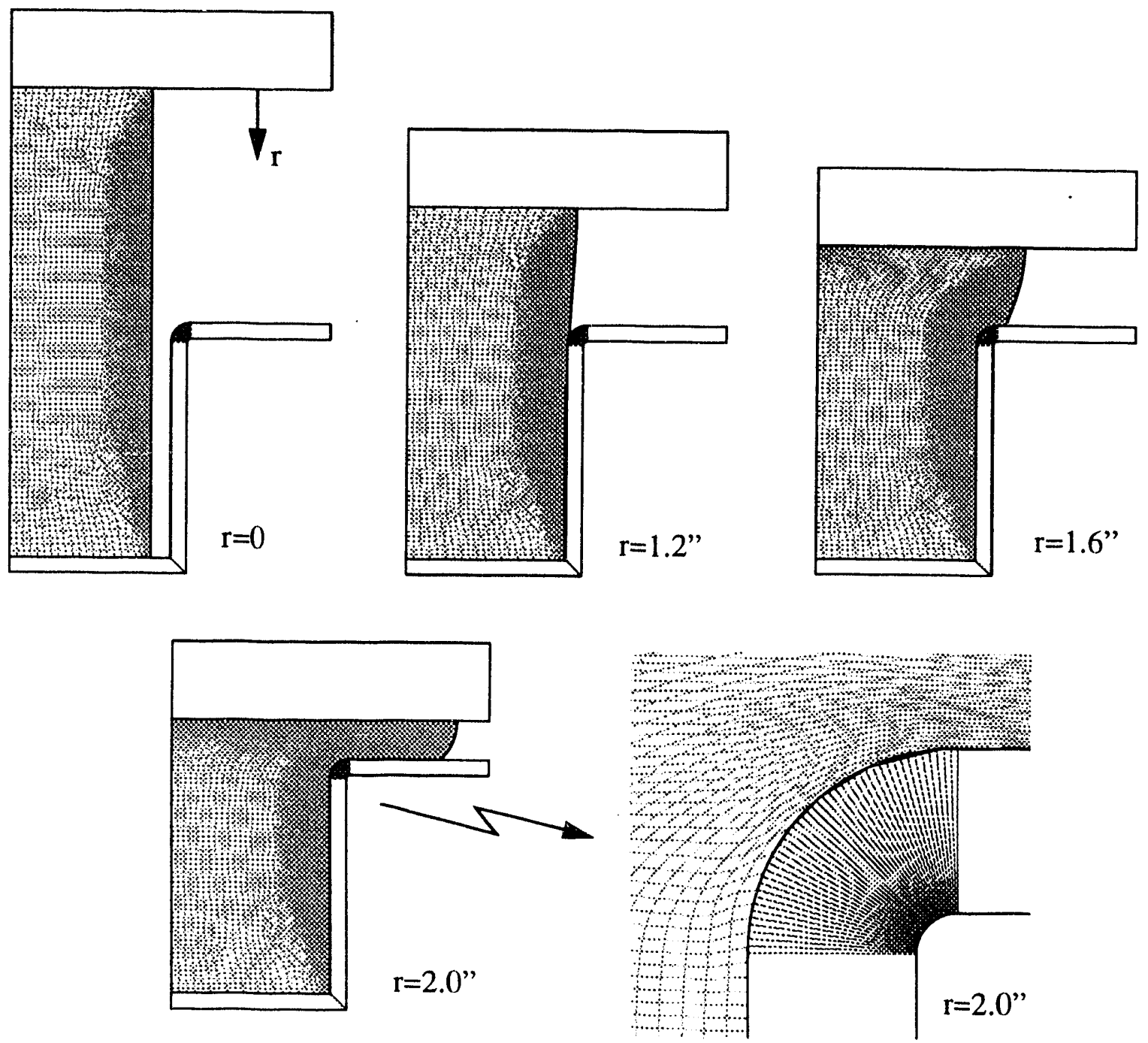

Figure 30. Rivet forging of a copper billet 


\section{Conclusions}

The current contact detection techniques used in the Sandia structural analysis codes have been reviewed. The review has pointed out several areas where improvements are necessary in current contact detection techniques. These areas include neighborhood identification and detailed contact checking. The neighborhood search should not rely on a closest master node to a slave node, and it should be independent of the problem geometry. In several cases, the detailed contact check requires improved accuracy in determining the contact point, penetration, and the pushback direction.

A new contact detection algorithm has been presented that offers improvements in these areas. The improvements are a result of a neighborhood identification strategy that uses a global contact search and a detailed contact check that distingushes between concave and convex surfaces. The key features of the new contact detection algorithm are:

- The efficient global search allows for global contact. This means that added capability for modelling self contacting structures and eroding or tearing surfaces is now available.

- The known locations of contacting surfaces and their velocities are used to construct a master surface capture box. This guarantees that only physically meaningful contacts are considered in the detailed contact check. The capture box also ensures that a minimum number of slave nodes are paired with the master surface.

- The position and velocity of both the slave node and master surface are considered in determining initial contact. This results in a physically correct determination of the contact location.

- A distinction between a concave and convex surface is made for slave nodes already in contact with a master surface. This results in a more accurate determination of the point of contact, amount of penetration, and the direction of pushback.

- An automatic surface definition algorithm allows for a simplified user input in many cases. One can, for example, include all surfaces of a body by specifying that the material be considered for global contact.

The capabilitites of the new contact detection algorithm have been demonstrated with several example problems. Several of the example problems have demonstrated improvements in the accuracy and efficiency of contact determination. Other problems have demonstrated the added capability provided by the new alogrithm. 
1 Taylor, L.M. and Flanagan, D.P., PRONTO2D: A Two-Dimensional Transient Solid Dynamics Program, SAND86-0594, Sandia National Laboratories, Albuquerque, NM 87185, 1987.

2 Taylor, L.M. and Flanagan, D.P., PRONTO3D: A Three-Dimensional Transient Solid Dynamics Program, SAND89-1912, Sandia National Laboratories, Albuquerque, NM 87185, 1989.

3 Attaway, S.W., Update of PRONTO2D and PRONTO3D Transient Solid Dynamics Programs, SAND90-0102, Sandia National Laboratories, Albuquerque, NM 87185, 1990.

4 Stone, C.M., SANTOS: A Two-Dimensional Finite Element Program for the Quasistatic Large Deformation, Inelastic Response of Solids, SAND90-0543, Sandia National Laboratories, Albuquerque, NM 87185, in preparation.

5 Biffle, J.H., JAC - A Two-Dimensional Finite Element Computer Program for the Nonlinear Quasi-Static Response of Solids with the Conjugate Gradient Method, SAND81-0998, Sandia National Laboratories, Albuquerque, NM 87185, 1984.

6 Biffle, J.H., JAC3D - A Three-Dimensional Finite Element Computer Program for the Nonlinear Quasi-Static Response of Solids with the Conjugate Gradient Method, SAND87-1305, Sandia National Laboratories, Albuquerque, NM 87185, in preparation.

7 Hallquist, J.O., User's Manual for DYNA2D: An Explicit Two-Dimensional Hydrodynamic Finite Element Code With Interactive Rezoning, Rev. 2, UCID-18756, Lawrence Livermore National Laboratories, 1984.

8 Hallquist, J.O. and Benson, D.J., User's Manual for DYNA3D: Nonlinear Dynamic Analysis of Structures, Rev. 3, UCID-19592, Lawrence Livermore National Laboratories, 1987.

9 Hibbitt, Karlsson and Sorensen, Inc., Contact Calculations with ABAQUS - ABAQUS Explicit Users Manual, Hibbitt, Karlsson and Sorensen, Inc., 1992.

10 Hallquist, J.O., NIKE2D: A Vectorized Implicit, Finite Deformation Finite Element Code for Analyzing the Static and Dynamic Response of 2-D Solids With Interactive Rezoning and Graphics, UCID-19677, Lawrence Livermore National Laboratories, 1986.

11 Hallquist, J.O., NIKE3D: An Implicit, Finite Deformation, Finite Element Code for Analyzing the Static and Dynamic Response of Three Dimensional Solids, UCID18822, Lawrence Livermore National Laboratories, 1984. 
Benson, B.J. and Hallquist, J.O., "A Single Surface Contact Algorithm for the PostBuckling Analysis of Structures," Computer Methods in Applied Mechanics and Engineering, Vol. 78, pp. 141-163, 1990.

13 Chaudhary, A.B. and Bathe, K.J., "A Solution Method for Static and Dynamic Analysis of Three-Dimensional Contact Problems with Friction," Computers and Structures, Vol. 24, No. 6, pp. 855-873, 1986.

14 Zhong, Z.H. and Nilsson, L., "A Contact Searching Algorithm for General 3D ContactImpact Problems," Computers and Structures, Vol. 34, No. 2, pp. 327-335, 1990.

15 Harding, D.C., Attaway, S.W., Neilsen, J., Blacker, T.D., and Pierce, J., "Evcluation of Four Multiple Package Crush Environment to the Common Package, Model I, Plutonium Air Transport Container," SAND92-0278, Sandia National Laboratories, Albuquerque, NM 87185, 1992.

16 Belytschko,T. and Lin, J.I., "A Three-Dimensional Impact-Penetration Algorithm with Erosion," Computers and Structures, Vol. 25, No. 1, pp. 95-104, 1987.

17 Belytschko,T. and Neal, M.O., "Contact-Impact by the Pinball Algorithm with Penalty and Lagrangian Methods," Int. J. Numerical Methods Eng., Vol. 31, pp. 547-572, 1991.

18 Metzinger, K., Attaway, S., and Mello, F., "Bobbin Stresses Generated by Wire Winding," First International Conference on Web Handling, Oklahoma State University, Stillwater, OK 74078, May 19-22, 1991.

19 Swegle, J.W., "Search Algorithm," Memo to Distribution, Sandia National Laboratories, Albuquerque, NM 87185, May 25, 1992.

20 Plimpton, S.J., "Molecular Dynamics Simulations of Short-Range Force Systems on 1024-Node Hypercubes," Proceedings of the Fifth Distributed Memory Computing Conference, Charleston, South Carolina, April 8-12, 1990.

21 Blacker, T. D., "FASTQ Users Manual Version 1.2," SAND88-1326, Sandia National Laboratories, Albuquerque, NM 87185, June 1988.

22 Sjaardema, G. D., "GJOIN: A program for Merging Two or More GENESIS Databases Version 1.4," SAND92-2290, Sandia National Laboratories, Albuquerque, NM 87185 , November 1992.

23 Gilkey, A. P. and Sjaardema, G. D., "GEN3D: A GENESIS Database 2D to 3D Transformation Program," SAND89-0485, Sandia National Laboratories, Albuquerque, NM 87185, March 1989.

24 Sjaardema, G. D., "GENSHELL: A GENESIS Database Shell Transformation Program," Sandia National Laboratories, Albuquerque, NM 87185, In preparation.

25 CRAY Research, Inc., Volume 3: UNICOS Math and Scientific Library Reference Manual, SR-2081 5.0, March 1989. 
26 Monaghan, J.J., “An Introduction to SPH," Comp. Phys. Comm., Vol. 48, pp. 89-98, 1988.

27 Harlow, F.H., "PIC and its Progeny," Comp. Phys. Comm., Vol. 48, pp. 1-10, 1988.

28 Stone, C.M., Wellman, G.W., and Krieg, R.D., A Vectorized Elastic/Plastic Power Law Hardening Material Model Including Luders Strain, SAND90-0153, Sandia National Laboratories, Albuquerque, NM 87185, March 1990. 


\section{A1 Appendix 1: Derivation of velocity based contact check}

This appendix derives the velocity based detailed contact check that determines the time and point of contact between a moving quadrilateral surface and a moving slave node in $3 \mathrm{D}$.

The equation of a triangular master surface, shown in Figure A1.1, can be written as:

$$
a\left(x-x_{1}\right)+b\left(y-y_{1}\right)+c\left(z-z_{1}\right)=0
$$

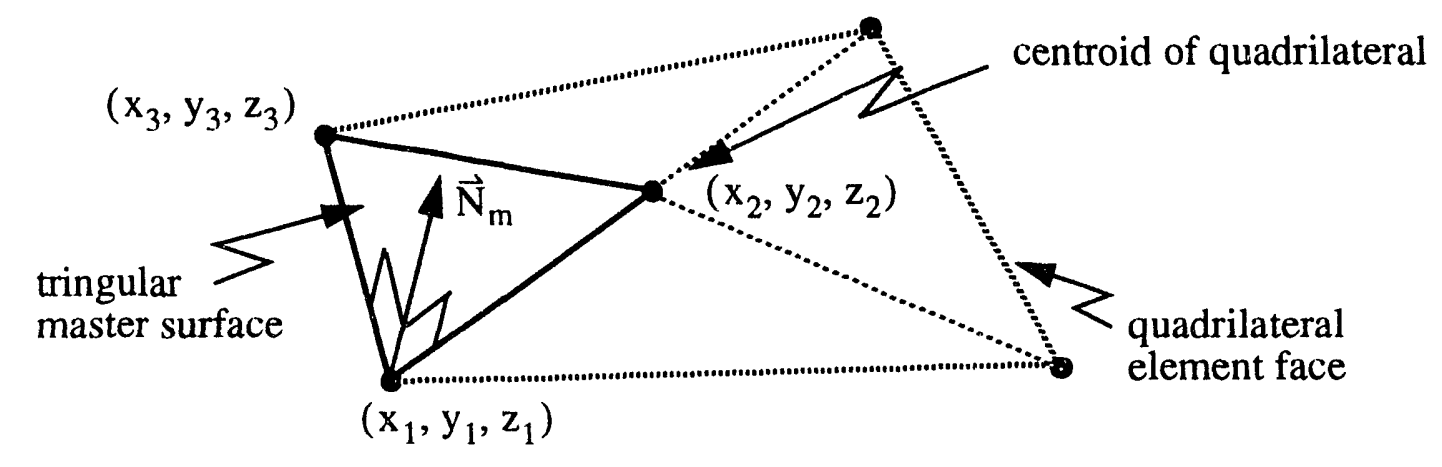

Figure A1.1 Triangular master surface definition on a quadrilateral element face

The point $x_{1}, y_{1}$, and $z_{1}$ is a node on the master surface and $a, b$, and $c$ are components of the master surface normal $\overrightarrow{\mathrm{N}}_{\mathrm{m}}=\mathrm{a} \grave{\mathrm{i}}+\mathrm{b} \hat{\mathrm{j}}+\mathrm{c} \overrightarrow{\mathrm{k}}$ :

$$
\begin{aligned}
& a=\left[\left(y_{1}-y_{3}\right)\left(z_{2}-z_{1}\right)-\left(y_{2}-y_{1}\right)\left(z_{1}-z_{3}\right)\right] \\
& b=\left[\left(x_{2}-x_{1}\right)\left(z_{1}-z_{3}\right)-\left(x_{1}-x_{3}\right)\left(z_{2}-z_{1}\right)\right] \\
& c=\left[\left(x_{1}-x_{3}\right)\left(y_{2}-y_{1}\right)-\left(x_{2}-x_{1}\right)\left(y_{1}-y_{3}\right)\right]
\end{aligned}
$$

Substituting equations (A1.2) in to equation (A1.1) and simplifying:

$$
\begin{aligned}
& \left\{x_{1} y_{3} z_{2}+x_{2} y_{1} z_{3}+x_{3} y_{2} z_{1}-x_{1} y_{2} z_{3}-x_{2} y_{3} z_{1}-x_{3} y_{1} z_{2}\right\} \\
& +\left\{\left(y_{1} z_{2}+y_{2} z_{3}+y_{3} z_{1}-y_{1} z_{3}-y_{2} z_{1}-y_{3} z_{2}\right) x\right. \\
& \quad\left(x_{1} z_{3}+x_{2} z_{1}+x_{3} z_{2}-x_{1} z_{2}-x_{2} z_{3}-x_{3} z_{1}\right) y \\
& \left.\quad\left(x_{1} y_{2}+x_{2} y_{3}+x_{3} y_{1}-x_{1} y_{3}-x_{2} y_{1}-x_{3} y_{2}\right) z\right\}=0
\end{aligned}
$$

Now, note that the nodal points of the triangular surface are moving, so that: 


$$
\begin{aligned}
& {\left[\left(x_{i}(t+\Delta t), y_{i}(t+\Delta t), z_{i}(t+\Delta t)\right)=\right.} \\
& \left.\quad\left(x_{i}(t)+\dot{x}_{i} \Delta t, \quad y_{i}(t)+\dot{y}_{i} \Delta t, \quad z_{i}(t)+\dot{z}_{i} \Delta t\right) \quad\right], i=1,3
\end{aligned}
$$

If equation (A1.4) is substituted into (A1.3), the result is an equation for a moving planar master surface, as follows:

$$
a_{0}+a_{1} x+a_{2} \cdot 1+a_{3} z=0
$$

where:

$$
\begin{aligned}
& \mathrm{a}_{0}=\left(\mathrm{x}_{1} \mathrm{y}_{3} \mathrm{z}_{2}\right)+\left(\mathrm{x}_{1} \mathrm{y}_{3} \dot{\mathrm{z}}_{2}+\mathrm{x}_{1} \dot{\mathrm{y}}_{3} \mathrm{z}_{2}+\dot{\mathrm{x}}_{1} \mathrm{y}_{3} \mathrm{z}_{2}\right) \Delta \mathrm{t} \\
& +\left(x_{1} \dot{y}_{3} \dot{z}_{2}+\dot{x}_{1} \ddot{y}_{3} \dot{z}_{2}+\dot{x}_{1} \dot{y}_{3} z_{2}\right) \Delta t^{2}+\left(\dot{x}_{1} \dot{y}_{3} \dot{z}_{2}\right) \Delta t^{3} \\
& +\left(\mathrm{x}_{2} \mathrm{y}_{1} \mathrm{z}_{3}\right)+\left(\mathrm{x}_{2} \mathrm{y}_{1} \dot{\mathrm{z}}_{3}+\mathrm{x}_{2} \dot{\mathrm{y}}_{1} \mathrm{z}_{3}+\dot{\mathrm{x}}_{2} \mathrm{y}_{1} \mathrm{z}_{3}\right) \Delta \mathrm{t} \\
& +\left(\mathrm{x}_{2} \dot{\mathrm{y}}_{1} \dot{\mathrm{z}}_{3}+\dot{\mathrm{x}}_{2} \mathrm{y}_{1} \dot{z}_{3}+\dot{\mathrm{x}}_{2} \dot{\mathrm{y}}_{1} \mathrm{z}_{3}\right) \Delta \mathrm{t}^{2}+\left(\dot{\mathrm{x}}_{2} \dot{\mathrm{y}}_{1} \dot{\mathrm{z}}_{3}\right) \Delta \mathrm{t}^{3} \\
& +\left(\mathrm{x}_{3} \mathrm{y}_{2} \mathrm{z}_{1}\right)+\left(\mathrm{x}_{3} \mathrm{y}_{2} \dot{\mathrm{z}}_{1}+\mathrm{x}_{3} \dot{\mathrm{y}}_{2} \mathrm{z}_{1}+\dot{\mathrm{x}}_{3} \mathrm{y}_{2} \mathrm{z}_{1}\right) \Delta \mathrm{t} \\
& +\left(\mathrm{x}_{3} \dot{\mathrm{y}}_{2} \dot{\mathrm{z}}_{1}+\dot{\mathrm{x}}_{3} \mathrm{y}_{2} \dot{\mathrm{z}}_{1}+\dot{\mathrm{x}}_{3} \dot{\mathrm{y}}_{2} \mathrm{z}_{1}\right) \Delta \mathrm{t}^{2}+\left(\dot{\mathrm{x}}_{3} \dot{\mathrm{y}}_{2} \dot{\mathrm{z}}_{1}\right) \Delta \mathrm{t}^{3} \\
& +\left(\mathrm{x}_{1} \mathrm{y}_{2} \mathrm{z}_{3}\right)+\left(\mathrm{x}_{1} \mathrm{y}_{2} \dot{\mathrm{z}}_{3}+\mathrm{x}_{1} \dot{\mathrm{y}}_{2} \mathrm{z}_{3}+\dot{\mathrm{x}}_{1} \mathrm{y}_{2} \mathrm{z}_{3}\right) \Delta \mathrm{t} \\
& +\left(\mathrm{x}_{1} \dot{\mathrm{y}}_{2} \dot{\mathrm{z}}_{3}+\dot{\mathrm{x}}_{1} \mathrm{y}_{2} \dot{\mathrm{z}}_{3}+\mathrm{x}_{1} \dot{\mathrm{y}}_{2} \mathrm{z}_{3}\right) \Delta \mathrm{t}^{2}+\left(\dot{\mathrm{x}}_{1} \dot{\mathrm{y}}_{2} \dot{\mathrm{z}}_{3}\right) \Delta \mathrm{t}^{3} \\
& +\left(\mathrm{x}_{2} \mathrm{y}_{3} \mathrm{z}_{1}\right)+\left(\mathrm{x}_{2} \mathrm{y}_{3} \dot{\mathrm{z}}_{1}+\mathrm{x}_{2} \dot{\mathrm{y}}_{3} \mathrm{z}_{1}+\dot{\mathrm{x}}_{2} \mathrm{y}_{3} \mathrm{z}_{1}\right) \Delta \mathrm{t} \\
& +\left(\mathrm{x}_{2} \dot{\mathrm{y}}_{3} \dot{\mathrm{z}}_{1}+\dot{\mathrm{x}}_{2} \mathrm{y}_{3} \dot{\mathrm{z}}_{1}+\dot{z}_{2} \dot{\mathrm{y}}_{3} \mathrm{z}_{1}\right) \Delta \mathrm{t}^{2}+\left(\dot{\mathrm{x}}_{2} \dot{\mathrm{y}}_{3} \dot{\mathrm{z}}_{1}\right) \Delta \mathrm{t}^{3} \\
& +\left(x_{3} y_{1} z_{2}\right)+\left(x_{3} y_{1} \dot{z}_{2}+x_{3} \dot{y}_{1} z_{2}+\dot{x}_{3} y_{1} z_{2}\right) \Delta t \\
& +\left(\mathrm{x}_{3} \dot{\mathrm{y}}_{1} \dot{\mathrm{z}}_{2}+\dot{\mathrm{x}}_{3} \mathrm{y}_{1} \dot{\mathrm{z}}_{2}+\dot{\mathrm{x}}_{3} \dot{\mathrm{y}}_{1} \mathrm{z}_{2}\right) \Delta \mathrm{t}^{2}+\left(\dot{\mathrm{x}}_{3} \dot{\mathrm{y}}_{1} \dot{\mathrm{z}}_{2}\right) \Delta \mathrm{t}^{3} \\
& \mathrm{a}_{1}=\left(\mathrm{y}_{1} \mathrm{z}_{2}\right)+\left(\mathrm{y}_{1} \dot{\mathrm{z}}_{2}+\dot{\mathrm{y}}_{1} \mathrm{z}_{2}\right) \Delta \mathrm{t}+\left(\dot{\mathrm{y}}_{1} \dot{\mathrm{z}}_{2}\right) \Delta \mathrm{t}^{2} \\
& +\left(\mathrm{y}_{2} \mathrm{z}_{3}\right)+\left(\mathrm{y}_{2} \dot{\mathrm{z}}_{3}+\dot{\mathrm{y}}_{2} \mathrm{z}_{3}\right) \Delta \mathrm{t}+\left(\dot{\mathrm{y}}_{2} \dot{\mathrm{z}}_{3}\right) \Delta \mathrm{t}^{2} \\
& +\left(\mathrm{y}_{3} \mathrm{z}_{1}\right)+\left(\mathrm{y}_{3} \dot{\mathrm{z}}_{1}+\dot{\mathrm{y}}_{3} \mathrm{z}_{1}\right) \Delta \mathrm{t}+\left(\dot{\mathrm{y}}_{3} \dot{\mathrm{z}}_{1}\right) \Delta \mathrm{t}^{2} \\
& +\left(\mathrm{y}_{1} \mathrm{z}_{3}\right)+\left(\mathrm{y}_{1} \dot{\mathrm{z}}_{3}+\dot{y}_{1} \mathrm{z}_{3}\right) \Delta \mathrm{t}+\left(\dot{\mathrm{y}}_{1} \dot{\mathrm{z}}_{3}\right) \Delta \mathrm{t}^{2} \\
& +\left(\mathrm{y}_{2} \mathrm{z}_{1}\right)+\left(\mathrm{y}_{2} \dot{\mathrm{z}}_{1}+\dot{\mathrm{y}}_{2} \mathrm{z}_{1}\right) \Delta \mathrm{t}+\left(\dot{\mathrm{y}}_{2} \dot{\mathrm{z}}_{1}\right) \Delta \mathrm{t}^{2} \\
& +\left(\mathrm{y}_{3} \mathrm{z}_{2}\right)+\left(\mathrm{y}_{3} \dot{\mathrm{z}}_{2}+\dot{\mathrm{y}}_{3} \mathrm{z}_{2}\right) \Delta \mathrm{t}+\left(\dot{\mathrm{y}}_{3} \dot{\mathrm{z}}_{2}\right) \Delta \mathrm{t}^{2}
\end{aligned}
$$




$$
\begin{aligned}
a_{2}= & \left(x_{1} z_{3}\right)+\left(x_{1} \dot{z}_{3}+\dot{x}_{1} z_{3}\right) \Delta t+\left(\dot{x}_{1} \dot{z}_{3}\right) \Delta t^{2} \\
& +\left(x_{2} z_{1}\right)+\left(x_{2} \dot{z}_{1}+\dot{x}_{2} z_{1}\right) \Delta t+\left(\dot{x}_{2} \dot{z}_{1}\right) \Delta t^{2} \\
& +\left(x_{3} z_{2}\right)+\left(x_{3} \dot{z}_{2}+\dot{x}_{3} z_{2}\right) \Delta t+\left(\dot{x}_{3} \dot{z}_{2}\right) \Delta t^{2} \\
& +\left(x_{1} z_{2}\right)+\left(x_{1} \dot{z}_{2}+\dot{x}_{1} z_{2}\right) \Delta t+\left(\dot{x}_{1} \dot{z}_{2}\right) \Delta t^{2} \\
& +\left(x_{2} z_{3}\right)+\left(x_{2} \dot{z}_{3}+\dot{x}_{2} z_{3}\right) \Delta t+\left(\dot{x}_{2} \dot{z}_{3}\right) \Delta t^{2} \\
& +\left(x_{3} z_{1}\right)+\left(x_{3} \dot{z}_{1}+\dot{x}_{3} z_{1}\right) \Delta t+\left(\dot{x}_{3} \dot{z}_{1}\right) \Delta t^{2} \\
a_{3}= & \left(x_{1} y_{2}\right)+\left(x_{1} \dot{y}_{2}+\dot{x}_{1} y_{2}\right) \Delta t+\left(\dot{x}_{1} \dot{y}_{2}\right) \Delta t^{2} \\
& +\left(x_{2} y_{3}\right)+\left(x_{2} \dot{y}_{3}+\dot{x}_{2} y_{3}\right) \Delta t+\left(\dot{x}_{2} \dot{y}_{3}\right) \Delta t^{2} \\
& +\left(x_{3} y_{1}\right)+\left(x_{3} \dot{y}_{1}+\dot{x}_{3} y_{1}\right) \Delta t+\left(\dot{x}_{3} \dot{y}_{1}\right) \Delta t^{2} \\
& +\left(x_{1} y_{3}\right)+\left(x_{1} \dot{y}_{3}+\dot{x}_{1} y_{3}\right) \Delta t+\left(\dot{x}_{1} \dot{y}_{3}\right) \Delta t^{2} \\
& +\left(x_{2} y_{1}\right)+\left(x_{2} \dot{y}_{1}+\dot{x}_{2} y_{1}\right) \Delta t+\left(\dot{x}_{2} \dot{y}_{1}\right) \Delta t^{2} \\
& +\left(x_{3} y_{2}\right)+\left(x_{3} \dot{y}_{2}+\dot{x}_{3} y_{2}\right) \Delta t+\left(\dot{x}_{3} \dot{y}_{2}\right) \Delta t^{2}
\end{aligned}
$$

The slave node is also moving, so that its position can be expressed as:

$$
\begin{aligned}
\left(x_{s}(t+\Delta t),\right. & \left.y_{s}(t+\Delta t), z_{s}(t+\Delta t)\right)= \\
& \left(x_{s}(t)+\dot{x}_{s} \Delta t, \quad y_{s}(t)+\dot{y}_{s} \Delta t, \quad z_{s}(t)+\dot{z}_{s} \Delta t\right)
\end{aligned}
$$

Following these definitions, we seek a time $\Delta t$ such that the slave node will be co-planar with the master surface. Such a time can be found (if it exists) by substituting equations (A 1.6), into equation (A1.5) and solving the following cubic equation in $\Delta \mathrm{t}$ :

$$
\mathrm{b}_{0}+\mathrm{b}_{1} \Delta \mathrm{t}+\mathrm{b}_{2} \Delta \mathrm{t}^{2}+\mathrm{b}_{3} \Delta \mathrm{t}^{3}=0
$$

where:

$$
\begin{aligned}
b_{0} & =x_{1}\left\{y_{2}\left(z_{s}-z_{3}\right)+y_{3}\left(z_{2}-z_{s}\right)+y_{s}\left(z_{3}-z_{2}\right)\right\} \\
& +x_{2}\left\{y_{1}\left(z_{3}-z_{s}\right)+y_{s}\left(z_{1}-z_{3}\right)+y_{3}\left(z_{s}-z_{1}\right)\right\} \\
& +x_{3}\left\{y_{s}\left(z_{2}-z_{1}\right)+y_{2}\left(z_{1}-z_{s}\right)+y_{1}\left(z_{s}-z_{2}\right)\right\} \\
& +x_{s}\left\{y_{1}\left(z_{2}-z_{3}\right)+y_{2}\left(z_{3}-z_{1}\right)+y_{3}\left(z_{1}-z_{2}\right)\right\}
\end{aligned}
$$




$$
\begin{aligned}
& b_{1}=x_{1}\left\{y_{2}\left(\dot{z}_{s}-\dot{z}_{3}\right)+y_{3}\left(\dot{z}_{2}-\dot{z}_{s}\right)+y_{s}\left(\dot{z}_{3}-\dot{z}_{2}\right)\right. \\
& \left.\dot{y}_{2}\left(z_{s}-z_{3}\right)+\dot{y}_{3}\left(z_{2}-z_{s}\right)+\dot{y}_{s}\left(z_{3}-z_{2}\right)\right\} \\
& +x_{2}\left(y_{1}\left(\dot{z}_{3}-\dot{z}_{s}\right)+y_{s}\left(\dot{z}_{1}-\dot{z}_{3}\right)+y_{3}\left(\dot{z}_{s}-\dot{z}_{1}\right)\right. \\
& \left.\dot{y}_{1}\left(z_{3}-z_{s}\right)+\dot{y}_{s}\left(z_{1}-z_{3}\right)+\dot{y}_{3}\left(z_{s}-z_{1}\right)\right\} \\
& +x_{3}\left(y_{s}\left(\dot{z}_{2}-\dot{z}_{1}\right)+y_{2}\left(\dot{z}_{1}-\dot{z}_{s}\right)+y_{1}\left(\dot{z}_{s}-\dot{z}_{2}\right)\right. \\
& \left.\dot{y}_{s}\left(z_{2}-z_{1}\right)+\dot{y}_{2}\left(z_{1}-z_{s}\right)+\dot{y}_{1}\left(z_{s}-z_{2}\right)\right\} \\
& +\mathrm{x}_{\mathrm{s}}\left(\mathrm{y}_{1}\left(\dot{z}_{2}-\dot{\mathrm{z}}_{3}\right)+\mathrm{y}_{2}\left(\dot{z}_{3}-\dot{\mathrm{z}}_{1}\right)+\mathrm{y}_{3}\left(\dot{z}_{1}-\dot{z}_{2}\right)\right. \\
& \left.\dot{y}_{1}\left(z_{2}-z_{3}\right)+\dot{y}_{2}\left(z_{3}-z_{1}\right)+\dot{y}_{3}\left(z_{1}-z_{2}\right)\right\} \\
& +\dot{x}_{1}\left\{y_{2}\left(z_{s}-z_{3}\right)+y_{3}\left(z_{2}-z_{s}\right)+y_{s}\left(z_{3}-z_{2}\right)\right\} \\
& +\dot{x}_{2}\left\{y_{1}\left(z_{3}-z_{s}\right)+y_{s}\left(z_{1}-z_{3}\right)+y_{3}\left(z_{s}-z_{1}\right)\right\} \\
& +\dot{x}_{3}\left\{y_{s}\left(z_{2}-z_{1}\right)+y_{2}\left(z_{1}-z_{s}\right)+y_{1}\left(z_{s}-z_{2}\right)\right\} \\
& +\dot{x}_{s}\left\{y_{1}\left(z_{2}-z_{3}\right)+y_{2}\left(z_{3}-z_{1}\right)+y_{3}\left(z_{1}-z_{2}\right)\right\} \\
& b_{2}=\dot{x}_{1}\left(y_{2}\left(\dot{z}_{s}-\dot{z}_{3}\right)+y_{3}\left(\dot{z}_{2}-\dot{z}_{s}\right)+y_{s}\left(\dot{z}_{3}-\dot{z}_{2}\right)\right. \\
& \left.\dot{y}_{2}\left(z_{s}-z_{3}\right)+\dot{y}_{3}\left(z_{2}-z_{s}\right)+\dot{y}_{s}\left(z_{3}-z_{2}\right)\right\} \\
& +\dot{x}_{2}\left(y_{1}\left(\dot{z}_{3}-\dot{z}_{s}\right)+y_{s}\left(\dot{z}_{1}-\dot{z}_{3}\right)+y_{3}\left(\dot{z}_{s}-\dot{z}_{1}\right)\right. \\
& \left.\dot{y}_{1}\left(z_{3}-z_{s}\right)+\dot{y}_{s}\left(z_{1}-z_{3}\right)+\dot{y}_{3}\left(z_{s}-z_{1}\right)\right\} \\
& +\dot{x}_{3}\left(y_{s}\left(\dot{z}_{2}-\dot{z}_{1}\right)+y_{2}\left(\dot{z}_{1}-\dot{z}_{s}\right)+y_{1}\left(\dot{z}_{s}-\dot{z}_{2}\right)\right. \\
& \left.\dot{y}_{\mathrm{s}}\left(\mathrm{z}_{2}-\mathrm{z}_{1}\right)+\dot{\mathrm{y}}_{2}\left(\mathrm{z}_{1}-\mathrm{z}_{\mathrm{s}}\right)+\dot{\mathrm{y}}_{1}\left(\mathrm{z}_{\mathrm{s}}-\mathrm{z}_{2}\right)\right\} \\
& +\dot{x}_{s}\left(y_{1}\left(\dot{z}_{2}-\dot{z}_{3}\right)+y_{2}\left(\dot{z}_{3}-\dot{z}_{1}\right)+y_{3}\left(\dot{z}_{1}-\dot{z}_{2}\right)\right. \\
& \left.\dot{y}_{1}\left(z_{2}-z_{3}\right)+\dot{y}_{2}\left(z_{3}-z_{1}\right)+\dot{y}_{3}\left(z_{1}-z_{2}\right)\right\} \\
& +x_{1}\left\{\dot{y}_{2}\left(\dot{z}_{s}-\dot{z}_{3}\right)+\dot{y}_{3}\left(\dot{z}_{2}-\dot{z}_{s}\right)+\dot{y}_{s}\left(\dot{z}_{3}-\dot{z}_{2}\right)\right\} \\
& +x_{2}\left\{\dot{y}_{1}\left(\dot{z}_{3}-\dot{z}_{s}\right)+\dot{y}_{s}\left(\dot{z}_{1}-\dot{z}_{3}\right)+\dot{y}_{3}\left(\dot{z}_{s}-\dot{z}_{1}\right)\right\} \\
& +x_{3}\left\{\dot{y}_{s}\left(\dot{z}_{2}-\dot{z}_{1}\right)+\dot{y}_{2}\left(\dot{z}_{1}-\dot{z}_{s}\right)+\dot{y}_{1}\left(\dot{z}_{s}-\dot{z}_{2}\right)\right\} \\
& +x_{s}\left\{\dot{y}_{1}\left(\dot{z}_{2}-\dot{z}_{3}\right)+\dot{y}_{2}\left(\dot{z}_{3}-\dot{z}_{1}\right)+\dot{y}_{3}\left(\dot{z}_{1}-\dot{z}_{2}\right)\right\} \\
& \mathrm{b}_{3}=\dot{\mathrm{x}}_{1}\left\{\dot{\mathrm{y}}_{2}\left(\dot{\mathrm{z}}_{\mathrm{s}}-\dot{\mathrm{z}}_{3}\right)+\dot{\mathrm{y}}_{3}\left(\dot{\mathrm{z}}_{2}-\dot{\mathrm{z}}_{\mathrm{s}}\right)+\dot{\mathrm{y}}_{\mathrm{s}}\left(\dot{\mathrm{z}}_{3}-\dot{\mathrm{z}}_{2}\right)\right\} \\
& +\dot{x}_{2}\left\{\dot{y}_{1}\left(\dot{z}_{3}-\dot{z}_{s}\right)+\dot{y}_{s}\left(\dot{z}_{1}-\dot{z}_{3}\right)+\dot{y}_{3}\left(\dot{z}_{s}-\dot{z}_{1}\right)\right\} \\
& +\dot{x}_{3}\left\{\dot{y}_{s}\left(\dot{z}_{2}-\dot{z}_{1}\right)+\dot{y}_{2}\left(\dot{z}_{1}-\dot{z}_{s}\right)+\dot{y}_{1}\left(\dot{z}_{s}-\dot{z}_{2}\right)\right\} \\
& +\dot{x}_{\mathrm{s}}\left\{\dot{y}_{1}\left(\dot{z}_{2}-\dot{z}_{3}\right)+\dot{y}_{2}\left(\dot{z}_{3}-\dot{z}_{1}\right)+\dot{y}_{3}\left(\dot{z}_{1}-\dot{z}_{2}\right)\right\}
\end{aligned}
$$


Suppose a solution for $\Delta t=\Delta t_{c}$ is found. Then it also must satisfy two conditions for it to be considered a contact. The first is that the time $\Delta t_{c}$ occurs in the next time step increment, $0 \leq \Delta \mathrm{t}_{\mathrm{c}} \leq \mathrm{dt}$. The other condition is that the contact point:

$$
\left(x_{c}, y_{c}, z_{c}\right)=\left(x_{s}+\dot{x}_{s} \Delta t_{c}, y_{s}+\dot{y}_{s} \Delta t_{c}, z_{s}+\dot{z}_{s} \Delta t_{c}\right)
$$

must lie inside the master surface edges. This can be determined exactly by computing the local $\xi, \eta$ coordinates of the contact point on the master surface. Figure A1.2 shows how an initial estimate $\xi_{0}, \eta_{0}$ of the local coordinates can be computed. Four triangles are constructed by connecting the contact point with the four corners of the quadrilateral master surface. The contact point is inside the master surface when all four areas $A_{1}, A_{2}, A_{3}$, and $A_{4}$ are all be positive. If it is inside the master surface, then an initial estimate of the local coordinates of the contact point can be found, as shown by the equations in Figure A1.2.

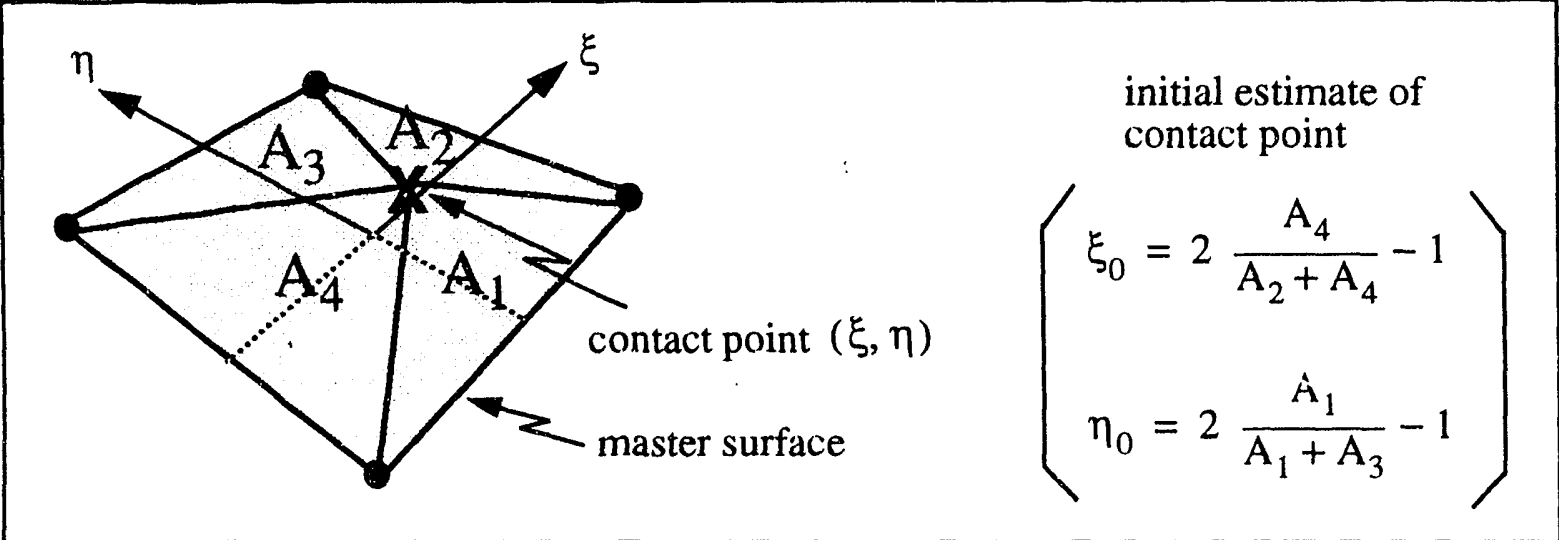

Figure A1.2 Initial estimates for the local coordinates of a contact point

The logic for these equations is based simply on the observation that $(\xi, \eta)=\left(\xi_{0}, \eta_{0}\right)$ is computed exactly for any rectangular quadrilateral and that the limiting values of $\xi$ and $\eta= \pm 1$ are computed exactly for any shaped quadrilateral. If the quadrilateral surface is a rectangle, then the proof is simple. If it is not, then by observation $\xi$ is computed exactly only when one of the areas $A_{2}$ or $A_{4}$ is zero. Likewise $\eta$ is computed exactly only when one of the areas $A_{1}$ or $A_{3}$ is zero. For example, suppose $A_{2}$ is zero then the estimated coordinate $\xi=\xi_{0}=1$ is exact. If $A_{4}$ is zero then the estimated coordinate $\xi=\xi_{0}=-1$ is also exact. If neither $\mathrm{A}_{2}$ or $\mathrm{A}_{4}$ are zero, then the equation gives a reasonable estimate of the contact point $\xi$. Improvements in the accuracy of the local coordinates can be achieved by performing Newton iterations on the nonlinear equations: 


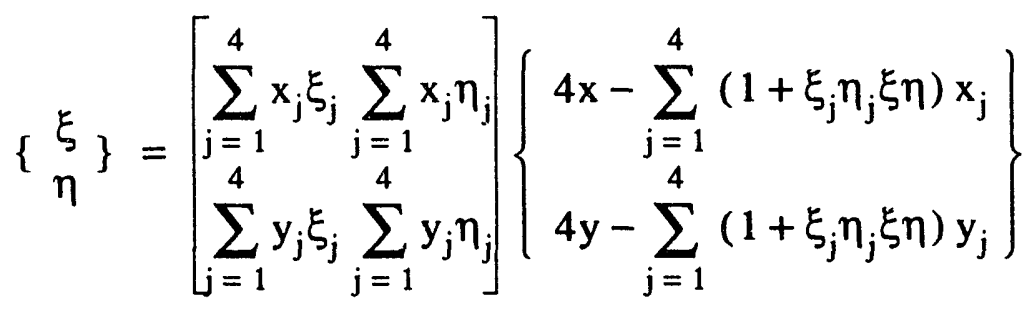

with the seed $(\xi, \eta)=\left(\xi_{0}, \eta_{0}\right)$. Upon converging on the local coordinates of the contact point $\xi, \eta$ with $\zeta=(+$ or -$) 1$, they should satisfy

$$
x_{c}=\sum_{j=1}^{8} N_{j}(\xi, \eta, \zeta) x_{j}, \quad y_{c}=\sum_{j=1}^{8} N_{j}(\xi, \eta, \zeta) y_{j}, \quad z_{c}=\sum_{j=1}^{8} N_{j}(\xi, \eta, \zeta) z_{j}
$$

where $N_{j}(\xi, \eta, \zeta), \quad j=1,8$ are the eight shape functions for a hexahedral element. 


\section{A2 Appendix 2: User instructions and example input files}

Reduced user input is a convenience that is an outcome of the global searching and automatic surface definition capability. One can, for example, include all surfaces of a body by specifying that the material be considered for global contact, or selectively include only a subset of the surface. Figure A2.1 shows the typical input required for a problem. Reducing the input required from the user minimizes the potential number of mistakes, which minimizes cost.

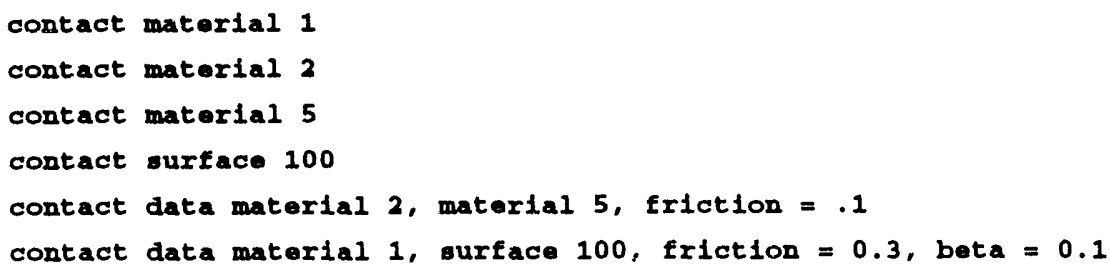

Surface 100 , materials 1,2 and 5 will be globally searched for contacts.

Contacts between material 2 and 5 will have a coefficient of friction equal to 0.1

Contacts between material 1 and contact surface 100 will have a coefficient of friction equal to 0.1 and a master slave partitioning of 0.1

Figure A2.1. Typical contact surface user input.

\section{A2.1 PRONTO User Instructions}

Listed below are updated and new keywords supported by the PRONTO 2D and 3D command language for using by the new contact detection algorithm. The uppercase letters represent the minimum abbreviation of each word.

- CONtact SURface

- CONtact MATerial

- CONtact DATa

The CONtact SURface command now has two distinct uses, a paired side set contact and a global contact. The paired side set contact is unchanged in its algorithm logic and usage. The global contact uses the new global contact detection algorithm and can be used to model a self contacting surface, for example. 


\section{A2.1.1 Paired Side Set Contact}

CONtact SURface, side 1 id, side 2 id, FIXED, $\beta$, toler side 1 id must match a side set on the GENESIS file side 2 id must match a side set on the GENESIS file FDXED -1 or the word FIXED will tie the contacts together $\beta \quad$ kinematic partition factor (default $=0.5$ ) toler tolerance for determining fixed contacts

CONtact SURface, side 1 id, side 2 id, $\mu_{0}, \beta, \mu_{1}, \gamma$ side 1 id must match a side set on the GENESIS file side 2 id must match a side set on the GENESIS file $\mu_{0} \quad$ static coefficient of friction (default $=0.0$ )

$\beta \quad$ kinematic partition factor (default $=0.5$ )

$\mu_{1} \quad$ high velocity coefficient of friction (default $=0.0$ )

$\gamma \quad$ velocity decay coefficient

A contact condition is enforced between the two surfaces defined by the respective side sets. The kinematic partition is a relative weighting of the master slave relationship of the two surfaces. A value of zero implies that the first surface (defined by side 1 id) acts only as a master and the second surface acts only as a slave. A value of one reverses these roles. The default value (0.5) gives a symmetric treatment of the contact. If one surface is much more massive than the other, this variable should be adjusted so that it is treated as the master. By massive, we mean that the surface either has a higher material density and/or a coarser mesh refinement.

\section{A2.1.2 Global Contact}

CONtact SURface, side 1 id side 1 id must match a side set on the GENESIS file

A contact condition is enforced between a surface contacting itself and other surfaces defined using the single side set CONtact SURface keyword and those surfaces defined using the CONtact MATerial keyword.

CONtact MATerial, material id 1

material 1 id must match a material id on the GENESIS file. If no material id is specified then all materials are included.

All surfaces associated with the material id are automatically determined and considered for contact with itself and other surfaces defined by the single side set CONtact SURface keyword 
and any additional surfaces defined as a result of repeated use of CONtact MATerial keyword. If the material id has an element death option, the surface will be automatically redefined $a s$ elements die. Note that the automatic surface redefinition is only done for those surfaces defined by the CONtact MATerial keyword and not those defined by the CONtact SURface keyword.

A new command called CONtact DATa is aciled so that friction and partitioning factors can be defined between contact surfaces and materials if required. Note that default values are set.

CONtact DATa, id 1, id $2, \mu_{0}, \beta, \mu_{1}, \gamma$

$\begin{array}{ll}\text { id } 1 & \text { must be either "SURface surface id" or "MATerial material id" } \\ \text { id } 2 & \text { must be either "SURface surface id" or "MATerial material id" } \\ \mu_{0} & \text { static coefficient of friction (default }=0.0 \text { ) } \\ \beta & \text { kinematic partition factor (default }=0.5 \text { ) } \\ \mu_{1} & \text { high velocity coefficient of friction (default }=0.0 \text { ) } \\ \gamma & \text { velocity decay coefficient }\end{array}$

All surfaces associated with the surface id/material id pair use the contact friction conditions and kinematic partitioning factor specified. If a part of a surface is multiply defined by a material id and a surface id, the contact data specification using the surface id will override the material id specification.

\section{A2.2 SANTOS and JAC User Instructions}

Both SANTOS and JAC have the partioned contact detection algorithm installed. At this moment contact surface pairs are still required (in the same manner outlined in the user instructions for the respective codes). The improvements in the accuracy of the detailed contact check are available in the updated versions of these codes. After the development of a partitioned contact enforcement algorithm, the benefit of using the global contact search can be available. 


\section{A2.3 Input files}

This section provides a collection of the FASTQ, GEN3D, GREPOS, GJOIN, and PRONTO (or SANTOS) inputs for the example problems presented in this report.

\section{A2.3.1 Contact of Elastic Blocks}

makefile:

bricks.g2d: brick.fsq brick.size

fastq -aprepro -m bricka.g2d brick.faq

brick.g: bricks.g2d brick.a1ze bricks.gen3d

gen3d -aprepro bricks.g2d brick.g <bricks.gen3d

brickl.g: brick.g brickl.grp brick.size

grepos -aprepro brick.g brickl.g<brickl.gIp

brick2.g: brick.g brick2.grp brick.81ze

grepos -aprepro brick.g brick2.g<brick2.grp

bricks.g: brick.g brick1.g brick2.g bricks.gjn

gjoin <bricks.gjn

FASTQ input:

title

contact test problem: two blocks impacting

\{include (brick.a1ze)\}

point 10.0 .

point 2 (xlen\} 0 .

point 3 (xlen\} (ylen\}

point 4 \{0.\} (ylen\}

line 1 str 120 \{1x\}

line 2 str 230 (1y)

line 3 str 340 \{ix\}

Iine 4 str 410 \{iy\}

$\begin{array}{lllllll}\text { region } & 1 & 1 & -1 & -2 & -3 & -4\end{array}$

sidebc 3001234

exit

brick.size:

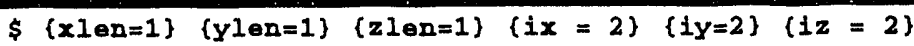

GEN3D file:

(include (brick.size))

translate $(1 z)$ (zlen\}

ssets back 100

ssets front 200

exit 
GREPOS file: brick 1:

(Include (brlck.size))

offset 0 . 0 . 0 .

change sideset 100101

change sideset 200201

change sideset 300301

exit

GREPOS file: brick 2:

\section{Case 1}

(include (brick.size) )

of fset $\left\{-0.9^{*} \times 1\right.$ en $\}$ (1.0*ylen) 0 .

change sideset 100101

change sideset 200201

change sideset 300301

exit

\section{Case 2}

(include (brick.size) \} offset $0 .\left(1.0^{*} y\right.$ len $\} 0$. change ideset 100101 change sideset 200201 change sideset 300301 exit

\section{GJOIN file:}

\section{brickl.g}

brick2.g

N

SSETS

COMBINE 101201301

COMBINB $102 \quad 202302$

BXIT

EXIT

No

bricks.g 
PRONTO3D input file:

TITH

EIXED CONTACT THST PROB

TERAIMATION TrTa $=.5 \mathrm{~B}-3$

PLOT TIMB $=.10-5$

OUTPOT TIME $=.1 \mathrm{e}-5$

MATRRIAL, 1, BLASTIC, .00074

YOUNGS MODULUS, 30E5

POISONS RATIO, . 3333

BND

MATBRIAL, 2, BLASTIC, .00074

YOUNGS MODULUS, $30 \mathrm{~B} 6$

POISONS RATIO, . 3333

BND

INITIAL VELOCITY MATERIAL $10 \ldots, 500 \ldots 0$.

INITIAL VELOCIMY MATBRIAL $20 \ldots, 0 \ldots, 0$.

PLOT BLEYMENT =

PLOT NODAL DISPLACEMRNT, REACTION, VELOCITY, ACCBLBRATION

CONTACT SURFACB 101

CONTACT SURPACE 102

BXIT 


\section{A2.3.2 Pressure Loading of Two Elastic Bodies}

FASTQ input:

titie

contact chatter

$\$\{r=.5\}(h \times=1\}\{h y=1\}$

point 10.0

point 2 ( $x) 0$.

point $3\{-r\} 0$.

line 1 atr 1205

line 2 atrc 23112

line 3 str 3105

region $1 \begin{array}{lllll}1 & -1 & -2 & -3\end{array}$

point 10 (r) $(0\}$

point $11(h x)\{0\}$

point $12(h x)(h y)$

point $13\{-b x\}$ (by)

point $14(-\mathrm{hx}\}$ (0)

point $15(-r\}\{0\}$

Iine 10 atr 101105

11ne 11 str $11 \quad 120$ 10

11ne 12 str 12130020

line 13 str 131400

line 14 str 141505

line 15 eirc $10 \quad 15 \quad 112$

region $2 \begin{array}{lllllll}2 & -10 & -11 & -12 & -13 & -14 & -15\end{array}$

cheme $1 \mathrm{c} 6 \mathrm{~s}$

scheme $2 x$

sidebc 115

sidebc 22

sidebc 10031

linebc 1112

exit

GLN3D file:

translate 101.

exit 
PRONTO3D input file:

ttitle

chatter toat problem

termination time .0025

plot time .00001

output time .00001

write restart .001

material 1 elastic .00074

youngs modulus 3006

polssons ratio .3

end

material 2 elastic .00074

youngs modulus $30 e 6$

poissons ratio . 3

end

contact aurface 12

pressure 100501.

function 50

0.0 .

.0001520000 .

.0120000 .

end

no displacement $Y 11$

no displacement $x 11$

exit 


\section{A2.3.3 Buckling of a Shell-like Structures}

makefile:

can.g2di can.fgq can.a1ze

fastq -aprepro -m can.g2d can.fag

can.g: can.g2d can.gen3d can.size

gen3d -aprepro can.g2d can.g <can.gen.2d

block.g2d: block.fsq can.size

fastq -aprepro -m block.g2d block.fsq

block.g: block.g2d block.gen3d can.size

gen3d -aprepro block.g2d block.g < block.gen3a

can_block.g: can.g block.g can_block.gjn

gjoln < can_block.gjn

FASTQ input for can:

\section{tit.le}

self contact test

(include (can.size))

point 100

point 2 (rad) 0 .

point 3 (rad+t\} 0 .

point 4 (-rad $\} 0$.

point 5 (-rad-t 0 .

line 1 str 230 (ithick\}

line 2 circ 241 (irad)

line 3 str 450 (ithick)

line 4 circ 351 (irad)

region $\begin{array}{llllll}1 & 1 & -1 & -2 & -3 & -4\end{array}$

line 5 circ 421 (irad)

line 6 circ 531 (irad)

\$ half can

region $\begin{array}{llllll}2 & 3 & -1 & -5 & -3 & -6\end{array}$

sidebc 125

sidebc 246 
FASTQ input for block:

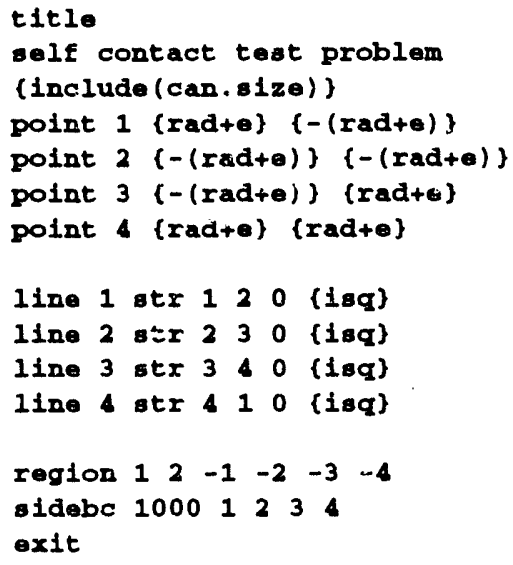

can.size:

\$ $\$$ cylinder $\mathrm{rad}=\{\mathrm{rad}=5\} \quad t=\{t=.2\}$ int $=\{$ ithlck $=3\} \quad\{$ rad $=40\}$

$\$$ length of $c /$ lender $(c y l l e n=15$ \}

$\$$ nuraber of elements in cylinder $\{i c y l=40\}$

$\$$ block $\{e=2\}$ int $=\{1 \mathrm{sq}=28\}$

$\$$ number of lements in block thick (iblkt $=5$ ) block thickness (b1kt =2.5)

$\$$ block angie (angle $=10$ )

GEN3D file for can:

(include (can.size)\}

translate (icyl) \{cyllen\}

sideset front 4

nodeset jiack 100

exit

GEN3D file for block:

\{include (can. a1ze)\}

translate (iblkt) (b1kt)

revolve $y(180+$ angle $)$

revcen 0.0 .0 .

offset $0.0 .(\operatorname{tand}(\operatorname{ang} l \theta) *(\operatorname{rad}+t)+.01)$

sideset front 3

exit

\section{GJOIN file:}

can.g

block.g

no

ssets

combine 124

combine 31000

exit

exit

no

can_block.g 
PRONTO3D input file:

title

self contact test problem

hourglass stiffness .01.03

TERMINATION TRas $=.004$

RHAD RESTART $=.002$

WRITE RESTART $=.00005$

PLOT TIME $=.0001$

OOTPOT TIME $=.00001$

MARRINI, 1, BIASTIC plast1c; .00074

YOUNOS MODULUS, $30 \mathrm{~B} 6$

POISONS RATIO, .3333

hardening modulus 0 .

ylelds strens 30000

beta $=1$

END

MATERIAL, 2, ELASTIC plagtic, .00074

YOUNGS MODULUS, $30 \mathrm{~B} 6$

POISONS RATIO, . .3333

hardening modulus 0 .

yields stress 30000

beta $=1$

BND

MATBRIAL, 3, BLAstic plastic, .00074

YOUNGS MODULUS, $30 \mathrm{E} 6$

POISONS RATIO, .3333

hardening modulus 0 .

yields atress 30000

beta $a=1$

BND

contact surface 1

contact ourface 3

INITIAL VELOCITY MATBRIAL $20 \ldots 0 .-5000$.

no displacemt $x 100$
no displacemt y 100
no displacemt z 100

PLOT BLEACNT =

PLOT NODAL DISPLACEABET, VBLOCITY, ACCELBRATION

plot state = oqpa

EXIT 


\section{A2.3.4 Cutting of a Steel Pipe}

makefile:

blade1.g2d: blade1.feq blade.aize

fastg -aprepro $-\mathrm{m}$ blade1.g2d blade1.fsq

blade1.g: blade1.g2d blade.eize blade1.gen3d

gen3d -aprepro blade1.g2d bladel.g <bladel.gen3d

edge.g2d: edge. fsq blade.size

fastq -aprepro -m edge.g2d edge.fsq

edge1.g3: edge.g2d blade..1ze edgel.gen3d

gen3d -aprepro edge.g2d edge1.g3< edge1.gen3d

edge1.g31: edge1.g3 edge1.grp blade..120

grepos -aprepro edge1.g3 edge1.g31 < edge1.grp

edge1.g: edge1.g31 edge11.grp blade.s1ze

grepos -aprepro edge1.g31 edgel.g < edge11.grp

edge2.g3: edge.g2d blade.size edge2.gen3d

gen3d -aprepro edge.g2d edge2.g3< edge2.gen3d

edge2.g31: edge2.g3 edge2.grp blade.size

grepos -aprepro edge2.g3 edge2.g31< edge2.gIP

edge2.g: edge2.g31 edge21.grp blade.s1ze

grepos -aprepro edge2.g31 edge2.g < edge21.grp

edge3.g3: edge.g2d blade.s1ze edge3.gen3d

gen3d -aprepro edge.g2d edge $3 . g 3$ < edge3.gen3d

edge3.g31: edge3.g3 edge3.grp blade.s1ze

grepos -aprepro edge $3 . g 3$ edge3.g31 < edge3.grp

edge3.g: edge3.g31 edge31.grp blade.size

grepos -aprepro edge3.g31 edge3.g < edge31.grp

edge4.g3: edge.g2d blade.81ze edge4.gen3d

gen3d -aprepro edge.g2d edge4.g3 < edge4.gen3d

edge4.g31: edge4.g3 edge4.grp blade.s1ze

grepos -aprepro edge4.g3 edge4.g31 < edge4.grp

edge4.g: edge4.g31 edge41.grp blade.size

grepos -aprepro edge4.g31 edge4.g < edge41.grp

edge5.g3: edge.g2d blade.size edge5.gen3d

gen3d -aprepro edge.g2d edge5.g3 < edge5.gen3d

edge5.g31: edge5.g3 edgp5.grp blade.81ze

grepos -aprepro edge5.g3 edge5.g31< edge5.gIP

edge5.g: edge5.g31 edge51.gxp blade.s1ze

grepos -aprepro edge5.g31 edge5.g< edge51.grp 
makefile (contd):

blade.g3: blade1.g edge1.g edge2.g edge3.g edge4.g edge5.g blade.gjn gjoin < blade.gjn

blade.g: blade.g3 blade.grp

grepos -aprepro blade.g3 blade.g < blade.grp

pipel.g2d: pipel.fsg blade.size pipe.size

fastg -aprepro -m pipe1.g2d pipe1.fsq

pipe1.g3: plpe1.g2d blade.size pipe.size pipel.gen3d

gen3d -aprepro pipe1.g2d pipe1.g3 <pipe1.gen3d

pipe1.g: pipe1.g3 pipe1.grp

grepos -aprepro pipel.g3 pipe1.g < pipel.grp

pipe2.g2d: pipe2.fsq blade.size pipe.size

fastq -apxepro -m p1pe2.g2d pipe2.fsq

pipe2.g3: plpe2.g2d blade.size pipe.size plpe2.gen3d

gen3d -aprepro pipe2.g2d pipe2.g3 <pipe2.gen3d

plfe2.g: plpez.g3 plpez.grp

grepos -aprepro pipe2.g3 pipe2.g < plpe2.grp

clamp.g2d: clamp.fsq blade.size pipe.size clamp.size

fastq -aprepro -m clamp.g2d clamp. foq

clamp.g3: clamp.g2d blade.size pipe.size clamp.size clamp.gen3d

gen3d -aprepro clamp.g2d clamp.g3 <clamp.gen3d

clamp.g: clamp.g3 blade.size clamp.size slamp.grp

grepos -aprepro clamp.g3 clamp.g < clamp.grp

pcut.g: blade.g pipe1.g pipe2.g clamp.g

gjoin < pcut.gjn 
FASTQ input for blade1:

\section{title}

pipe cutter blade

(include (blade.size) )

point $1-2.594 \quad 0.312$

point 22.7160 .312

point $3\left\{x_{3}\right\}\left\{y^{3}\right\}$

point $4\left\{x_{4}\right\}$ (y4)

point $5\{x 5\}\{y 5\}$

point $6(x 6)\{y 6\}$

point $7\{x 7\}\{y 7\}$

point $8\left\{x^{8}\right\}\left\{Y^{8}\right\}$

point 9 (x9) $\{y 9\}$

point $10\{0.5 *(x 8+x 9)\}\left\{y^{8}+0.7 * a b_{8}\left(x^{8}-x^{\prime} 9\right)\right\}$

point $11(0.5 *(x 8+x 9)\}\left\{y^{8}-0.068\right\}$

line 1 atr $120 \quad$ (3*11)

line 2 str 230 (4*i1\} 0.9

line 3 str $340 \quad\{2 * 11\}$

line \& str \&8 0 \{10*11\} 0.8

line 5 cire 11810 (3*11) 1.17647

line 6 circ $91110\{3 * 11\} 0.85$

line 7 str 960 (10*i1\} 1.25

line 8 str $670 \quad(3 * i 1)$

line 9 str 170 (4*11\} 0.9

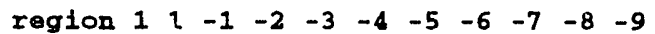

scheme 1 X6s

nodebc 2001

exit

GEN3D file for blade 1:

(Include (blade.size) )

translate (12) (bthick/2.+.002)

zsets back 100

seets front 20

$\operatorname{exit}$

blade.size:

$\$\{11=1\} \quad\{12=2\}$ (bthick $=0.25\}$

$\$\left\{x^{3}=2.716\right\} \quad\left\{y^{3}=-2.1715\right\}$

$\$\{x 4=1.5\} \quad(y 4=-2.286\}$

$\$\{x 5=0.156\}\{y 5=-3.63\}$

$\$\{x 6=-1.0409\} \quad\{y 6=-2.4331\}$

$\$\{x 7=-2.594\}\{y 7=-2.175\}$

$\$\{x 8=0.288583\} \quad\{y 8=-3.497417\}$

$\$\{x 9=0.023417\} \quad\{y 9=-3.497417\}$ 
FASTQ input for edge:

title

pipe cutter blade edge

(include (blade.size))

point 10.0020 .0

polnt $2-0.1250 .0$

point $3-0.02-0.2$

point $40.002-0.2165$

point $50.002-0.205$

Iine 1 atr 120 (12)

IIne 2 str 230 (2*12\} 0.8

line 3 circ 3452

line 4 str $410\{2 * 12\} 1.4$

region $1 \begin{array}{lllll}1 & -1 & -2 & -3 & -4\end{array}$

scheme $1 \times 6$

nodebc 1004

aldebc 1023

exit

GEN3D file for edge 1:

(include (blade.size))

translate $(2 * 11),(x 3-x 4)$

axit

GREPOS files for edge1:

[include (blade.size)]

offset apline

slope left $\left\{a t a n\left(a b s\left(y 4-y^{3}\right) / a b s\left(x 4-x^{3}\right)\right)\right\}$

slope right (atan $\left.\left(\operatorname{abs}\left(y^{4}-y^{3}\right) / a b s\left(x 4-x^{3}\right)\right)\right\}$

0.00 .0

$\left\{-a b_{B}\left(x_{4}-x^{3}\right)\right\} 0.0\left\{-a b_{B}\left(y^{3}-y^{4}\right)\right\}$

end

exit

(1nclude (blade.size)\}

revcen $0.0 \quad 0.0 \quad 0.0$

revolve $Y 90$.

offset $x\left(x_{3}\right) y\left(y^{3}\right)=\{-b t h 1 c k / 2.0\}$

exit

GEN3D file for edge2:

(1nclude (blade.size) \}

translate $(10 * 11\},(x 4-x 8) 0.8$

exit 
GREPOS files for edge2:

(1nclude (blade.size)\}

offaet spline

- lope left (atan (abs $\left(y^{5}-y^{4}\right)$ / abs (x5-x4))\}

- lope right (atan (abs (y5-y4)/abs (x5-x4))\}

0.00 .0 .

$\{-a b(x 5-x 4)\} 0.0\left\{-a b s\left(y 4-y^{5}\right)\right\}$

end

exit

(Include (blade.size)\}

revcen $0.0 \quad 0.0 \quad 0.0$

revolve y 90 .

offsot $x\left\{x^{4}\right\}$ y $\left(y^{4}\right\} z\{$-bthick $/ 2.0\}$

exit

GEN3D file for edge3:

(Include (blade.size)\}

translate $\{10 * 11\},\{x 9-x 6\} 1.25$

exit

GREPOS files for edge3:

\{1nclude (blade.size) \}

offset spline

- lope left (atan (abs $(y 6-y 5) / a b s(x 6-x 5))$ )

slope right (atan (abs $(y 6-y 5) / a b s(x 6-x 5))$ \}

0.00 .0 .

$\left\{-a b_{8}\left(x 6-x^{2}\right)\right\} \quad 0.0 \quad\left(a b_{B}\left(y 5-y^{6}\right)\right\}$

end

exit

(Include (blade.size))

revcen $0.0 \quad 0.0 \quad 0.0$

revolve $Y 90$.

offset $x\{x 9\} y$ ix 9$\} z\{-b t h 1 c k / 2.0\}$

exit

GEN3D file for edge4:

(1nclude (blade.size) )

translate $\{3 * 11\},(x 6-x 7\}$

exit 
GREPOS files for edge4:

(include (blade.size) )

offset spline

slope left [atan (abs $\left.\left(y 7-y^{6}\right) / a b s\left(x^{7}-x 6\right)\right)$ \}

slope right (atan(abs $\left.\left(y 7-y^{6}\right) / a b s\left(x^{7}-x^{6}\right)\right)$ )

0.00 .0 .

$\left\{-\operatorname{abs}\left(x^{2}-x_{6}\right)\right\} 0.0 \quad\left\{a b s\left(y 6-y^{7}\right)\right\}$

end

exit

(include (blade.size) \}

revcen $0.0 \quad 0.0 \quad 0.0$

revolve $y 90$.

offset $x\left\{x^{6}\right\}$ y $\left(y^{6}\right\}$ z $($-bthick/2.0

exit

GEN3D file for edge5:

(include (blade.size)]

translate $\left\{66^{*} i 1\right\},\{a b s(x 8-x 9)\}$

exit

GREPOS files for edge5:

(include (blade.size))

offset spline

slope top 0.0 \{atan (abs $\left.\left.\left(y^{5}-y^{4}\right) / a b s\left(x^{5}-x^{4}\right)\right)\right\}$

slope bottom $0.0\left\{-a t a n\left(a b s\left(y 5-y^{4}\right) / a b s(x 5-x 4)\right)\right\}$

$0.0 \quad 0.0 \quad 0.0$

$\$\{a b s(x 8-x 9) / 4.0\} \quad 0.0-0.048083261$

$(-a b s(x 8-x 9) / 2.0\} 0.0-0.068$

$\$\{3.0 * a b s(x 8-x 9) / 4.0\} \quad 0.0-0.048083261$

$\{-\operatorname{abs}(x 8-x 9)\} \quad 0.0 \quad 0.0$

end

exit

(include (blade.size) )

revcen $0.0 \quad 0.0 \quad 0.0$

revolve $y 90$.

offset $x\left\{x^{8}\right\}$ y $\left(y^{8}\right\} \geq\{$-bthick/2.0\}

exit 
GJOIN file for blade:

blade1.g

edge1.g

combine .000001

combine exit

exit

yes

edge2.g

combine .000001

combine exit

exit

Yes

edge 3.g

combine .000001

combine exit

exit

Yes

edge4.g

combine .000001

combine exit

exit

Yes

edge $5 . g$

combine .01

combine exit

exit

no

blade.g3

GREPOS file for blade:

offset $y-0.064$

exit 
FASTQ input for pipe1:

title

pipe

(1nclude (pipe.size)

(include (blade. $.1 z \bullet$ ) \}

point $1(x 5)(y 5-(b t h 1 c k / 2.) / \tan (3.14159 * 30 . / 180)-.p x-p t)$

point 2 (x5) $(y 5-(b t h 1 c k / 2.) / \tan (3.14159 * 30.1180)-$. pt $)$

point 3 (x5) (y5 - (bthlck/2.)/tan(3.14159*30.1180.))

point $(x 5)(y 5-(b t h 1 c k / 2.) / \tan (3.14159 * 30.1180)-.2 * p r-p t)$

point 5 (x5) $(y 5-(b t h i c k / 2.) / \tan (3.14159 * 30.1180)-.2 * p r-2 * p t)$

11ne 1 str 230 (ithick)

line 2 circ $2 \& 1$ (irad)

line 3 str 40 (ithick)

line 4 circ 351 (1rad)

1ine 5 circ 41 (irad)

line 6 circ 531 (irad)

region $\begin{array}{llllll}1 & 2 & -1 & -2 & -3 & -4\end{array}$

$\begin{array}{lllllll}\text { region } & 2 & 3 & -1 & -5 & -3 & -6\end{array}$

exit

pipe.size:

\$ (1thlck=4) (1rad=50) $(\mathrm{pr}=1.0335\} \quad(\mathrm{pt}=0.154)$

\$ $\{$ th hick2 $=2\} \quad\{$ irad2 $=25\}$

$\$$ (plength $=1.5\} \quad\{13=16\}$

$\$$ (plength2 $=3.5\} \quad\{i 4=12\}$

GEN3D file for pipe 1:

[include (blade.120)\}

(include (plpe.size)]

translate (13\} \{plength/2.\} 1.05

nsets front 100

ssets back 400

oxit 
GREPOS file for pipe1:FASTQ input for pipe2:

(include (blade. size)

revcen $\{x 5\} \quad 0.0 \quad 0.0$

revolve $y 180$

offoet $\times 0.00001=\{-$ bthick $/ 2++0.0001\}$

exit

titlo

pipe

(include (pipe.size))

(include (blade.size) )

point $1\{x 5\}$ (y5 - (bthick/2.)/tan(3.14159*30./180.) - pr - pt $\}$

point $2\{\times 5\}\{y 5$ - (bthick/2.)/tan(3.14159*30.1180.) - pt $\}$

point $3\{x 5\}\{y 5-($ bthick/2.) $/ \tan (3.14159 * 30 . / 180)\}$.

point $4\{x 5\}(y 5-(b t h i c k / 2.) / \tan (3.14159 * 30.1180)-.2 * p r-p t)$

point $5\{x 5\}\left\{y^{5}-(\right.$ bthick/2.)/tan $(3.14159 * 30 . / 180)-.2 \star p r-2 \star p t\}$

line 1 str 230 \{1thick2\}

line 2 circ $2 \& 1$ \{irad2\}

line 3 st 450 (1thick2)

line 4 circ 351 \{1rad2\}

1ine 5 circ 421 \{1rad2\}

line 6 circ $5 \quad 3 \quad 1$ \{irad2\}

region $\begin{array}{llllll}1 & 4 & -1 & -2 & -3 & -4\end{array}$

region $2 \begin{array}{llllll}2 & 5 & -1 & -5 & -3 & -6\end{array}$

exit

GEN3D file for pipe2:

(include (blade.size)

(include (pipe.size)\}

translate (14\} (plength2) 1.08

ssets front 401

exit

GREPOS file for pipe2:

\{include (blade.size)\}

\{include (pipe.size)\}

revcen $\{x 5\} \quad 0.0 \quad 0.0$

revolve y 180

offset $\times 0.00001 \mathrm{z}$ (-bthick $/ 2+0.0001+$ plength $/ 2$.

exit 
FASTQ input for clamp:

title

clamp

(Include (pipe.size)\}

\{include (blade.size)\}

(include (clamp.size)

point $1-4.0(y 5-(b t h i c k / 2.) / \tan (3.14159 * 30 . / 180)-.2 * p x-2 * p t)$

point $24.0\{y 5-(b t h 1 c k / 2.) / \tan (3.14159 * 30.1180)-.2 * p r-2 * p t\}$

point $34.0\left(y 5-(b t h 1 c k / 2.) / \tan (3.14159 * 30 . / 180)-.2{ }^{*} p r-2{ }^{*} p t-c h e i g h t\right\}$

point $4-4.0\left(y 5-(b t h i c k / 2\right.$. $) / \tan (3.14159 * 30.1180)-.2 * \mathrm{pr}-2{ }^{*} \mathrm{pt}$ - cheight $)$

line 1 str 1200

line 2 str 23002

line 3 stt 340 8

line 4 atr 4102

region $\begin{array}{llllll}1 & 1 & -1 & -2 & -3 & -4\end{array}$

nodebc 3003

sidebc 301

exit

clamp.size:

$\$\{$ cthick $=0.5\} \quad$ (coffset $=0.31\}$

$\$$ (choight $=1.0$ \}

GEN3D file for clamp:

(include (blade.size)

\{include (pipe.size)\}

(include (clamp. size))

translate 2 \{cthick\}

sset back 31

exit

GREPOS file for clamp:

(include (blade.size)

\{include (clamp.size)\}

offset $z$ (cthick+coffset-bthick/2.)

exit 
GJOIN file for blade, pipe, and clamp:

\section{blade.g}

pipe1.g

no

exit

Yes

pipe2.g

no

exit

yes

clamp. 9

no

exit

no

pout.g

PRONTO3D input file:

TITLE

Plpe cutting simulation

\$hourglass stiffness $0.01 \quad 0.03$

TRRMINATION TIMB $=4 \cdot \mathrm{E}-3$

PLOT TIME $=4 . \theta-5$

OUTPUT TIMB $=4.0-5$

write restart $=8.0-5$

function 1

0.01 .0

1.01 .0

end

MATERIAL， 1， BLASTIC,

.00074

YOUNGS MODULUS, 30B6

POISONS RATIO, . .3333

BND

MATBRIAL, 2, plh strength ,

.00074

YOUNGS MODULUS, 3086

POISONS RATIO, .3

yield stress, 38.803

hardening constant $93.6 e 3$

hardening exponent 0.4539

luders strain $0 . \cdots 1$

failure value 1.27

decay constant 0.7

END

MATBRIAL, 3, plh strength, .00074

YOUNGS MODULUS, $30 \mathrm{~B} 6$

POISONS RATIO, . 3

yleld stress, 38.833

hardening constant 93.683

hardening exponent 0.4539

luders strain 0.021

failure value 1.27

decay constant 0.7

BND 
PRONTO3D input file (contd):

MATERIAL, 4, plb strongth, .00074

YOUNas MODULUS, $30 \mathrm{~B} 6$

POISONS RATIO, . 3

Yleld stress, 38.803

hardening constant 93.603

hardening exponent 0.4539

luders train 0.021

fallure value 1.27

decay constant 0.7

END

MatrerIAI, 5, plh strength, .00074

YOUNas MODULUS, 30B6

POISONS RATIO, . 3

yield atress, 38.803

hardening constant 93.603

hardening exponent 0.4539

luders train 0.021

fallure value 1.27

decay constant 0.7

BND

death 2 decay min 0.1

death 3 decay min 0.1

prescribed VELOCIFY Y $2001-1187.5$

INITIAL VRLOCITY MATERIAL $10 .-1187.50$.

no displacement 2100

no displacoment y 300

PLOT BLEMRNT = vonmises, pressure

plot state = eqps

PLOT NODAL DISPLACEMENT, REACTION, VELOCITY, ACCBLERATION

contact aurface $400 \quad 401$ fixed

CoNrAcT matertal 1

CONTACT material 2

Conracr material 3

BXIT 


\section{A2.3.5 Elastic-Plastic Bar Impacting Bricks}

\section{makefile:}

bricks.g2d: bricks + fsq

fasta -aprepro -m bricks.g2d -- bricks.fsq

bricka.g: bricks.g2d bricks.gen3d

gen3a bricks.g2d bricke.g <bricks.gen3d

bricks.gx; bricks.g

exoxdr bricks.g bricks.gx

wall.g2d: wall.fag

fastq -m wall.g2d - wall.fsq

wall.g: wall.g2d wall.gen3d

gen3d wall.g2d wall.g <wall.gen3d

brick_wall.gx: brick_wall.g

exoxdx brick_wall.g brick_wall.gx

rod.g2d: rod. $f a q$

fastq $-m$ rod.g2d -- rod.faq

rod.g: rod.g2d rod.gen $3 d$

gen3d rod.g2d rod.g <rod.gen3d

brick_wall.g: bricks.g wall.g rod.g

gjoin < brick_wall.gjn 
FASTQ file for bricks:

TITLB

STREL ROD HITTINO BRICRS

$\$\{0=.005\}$

POINT $1(0 .+\infty)(0 .+e)$

POINT 2 (1.-e) $(0 .+\infty)$

POINT $3(1,-0) \quad(.5-\theta)$

POINT $4(0 .+\theta)(.5-\theta)$

LINE 1 STR 122010

LINE 2 STR 23005

LINB 3 STR 34010

LINE \& STR \& 105

RBGION $\begin{array}{llllll}1 & 1 & -1 & -2 & -3 & -4\end{array}$

POINT $11(1,+\infty)\{0 .+\theta\}$

POINT $12\{2 .-\theta\}(0 .+e\}$

POINT $13(2 .-0) \quad(.5-0)$

POINT $14(1 .+\theta)(.5-\theta)$

LIMR 11 STR 11120010

LINB 12 STR 12 13 005

LINB 13 STR $\begin{array}{lllll}13 & 14 & 0 & 10\end{array}$

LINB 14 STR 141105

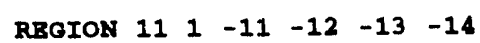

POINT $21(2 .+\theta)(0 .+\theta\}$

POINT $22(3 .-\theta)(0 .+\theta\}$

POINT $23(3 .-0)\{.5-\theta\}$

POINT $24(2 .+0)(.5-0)$

IINE 21 STR $2122 \quad 0 \quad 10$

LINB 22 STR $22 \quad 23005$

LINE 23 STR 23 24 0 O 10

LINB 24 STR 2421005

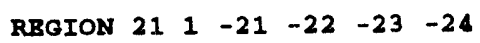

\$ SBCOND ROV

POINT $101(0 .+0)(j+\theta)$

POINT $102(.5-\theta)(0.5+\theta\}$

POINT 103 (.5-e) (1.-e)

POINT $104(0 .+e)(1 .-6)$

LINB 101 STR 10110205 LINR 102 STR $102 \quad 103 \quad 05$

LINE 103 STR $103 \quad 10405$

LINE 104 STR 10410105

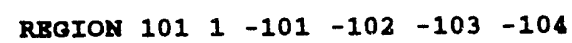

POINT $111(.5+0\}\{.5+\theta\}$

POINT 112 (1.5-0) $(.5+\theta)$

POINT 113 \{1.5-e\} (1. $\Leftrightarrow$ \}

POINT $114\{.5+\infty)(1,-6\}$

LINE 111 STR 111112010

IINE 112 STR 11211305

LINB 113 STR $113 \quad 11400$

LINB 114 STR 11411105

RBGION $\begin{array}{llllll}111 & 1 & -111 & -112 & -113 & -114\end{array}$

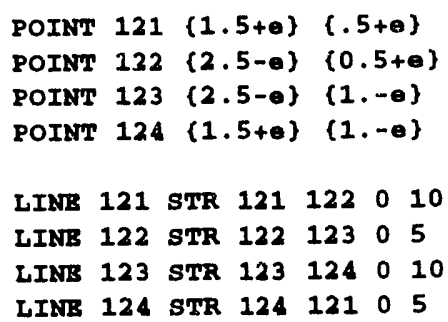


FASTQ file for bricks (contd):

LINB 211 STR 211212010

IINB 212 STR $212 \quad 213 \quad 0 \quad 5$

IINE 213 STR 2132140010

IINE 214 STR 21421105

RBGION $211 \quad 1 \quad-211 \quad-212 \quad-213 \quad-214$

POINT $221\{2,+\theta\}\{1,+\theta\}$

POINT 222 (3. $-\theta)$ (1. + $+\theta)$

POINT 223 (3.-e\} $\{1.5-\theta\}$

POINT $224(2 .+\theta)(1.5-\theta)$

IINR 221 STR 221222010

LINE 222 STR $222 \quad 223 \quad 0 \quad 5$

LINE 223 STR 22322400

LINE 224 STR 22422105

REGION $221 \quad 1 \quad-221 \quad-222 \quad-223 \quad-224$

\$ FOURTH ROW

POINT $301(0 .+e\}\{1.5+\theta\}$

POINT 302 (.5-e $\{1.5+e\}$

POINT $303 \quad(.5-\theta\}\{2.0-\theta\}$

POINT $304\{0 .+\theta\}\{2.0-\theta\}$

LINE 301 STR 30130205

LINE 302 STR $302 \quad 303 \quad 0 \quad 5$

LINE $303 \quad$ STR $303 \quad 304 \quad 0 \quad 5$

LINE 304 STR 30430105

REGION $301 \quad 1 \quad-301 \quad-302 \quad-303 \quad-304$

POINT $311\{.5+\theta\} \quad 1.5+\theta\}$

POINT $312\{1.5-\theta\} \quad\{1.5+\theta\}$

POINT $313\{1.5-\theta\} \quad(2 .-\theta)$

POINT $314\{.5+\theta\}(2 .-\theta)$

LINE 311 STR 3113120010

LINE 312 STR 31231300

LINE 313 STR $313 \quad 3140010$

LINE 314 STR 31431105

RBGION $311 \quad 1-311-312 \quad-313-314$

POINT $321\{1.5+\theta\} \quad(1.5+\theta\}$

POINT 322 (2.5-e $\quad(1.5+\theta\}$

POINT $323(2.5-\theta) \quad(2 .-\theta)$

POINT $324(1.5+\theta)(2 .-\theta\}$

LINE 321 STR $321322 \quad 0 \quad 10$

LINB 322 STR 32232305

LINB 323 STR $323 \quad 324 \quad 0 \quad 10$

T.TNR 32.4 STR $22.4 \quad 32.105$

RBGION $\begin{array}{llllll}321 & 1 & -321 & -322 & -323 & -324\end{array}$

POINT $331\{2.5+\theta\} \quad\{1.5+\theta\}$

POINT 332 (3.0-e $\{1.5+e\}$

POINT $333\{3.0-\theta\}\{2 .-\theta\}$

POINT $334\{2.5+\theta\}\{2 .-\theta\}$

LINE 331 STR $331 \quad 332 \quad 0 \quad 5$

LINE 332 STR $332 \quad 333005$

LINE $333 \quad$ STR $333 \quad 334 \quad 0 \quad 5$

LINE 334 STR $334 \quad 331 \quad 0 \quad 5$

REGION $331 \quad 1 \quad-331 \quad-332 \quad-333 \quad-334$

\$ FIFTH ROW

$\begin{array}{llll}\text { POINT } 401 & \{0 .+e\} & \{2,+\theta\} \\ \text { POINT } 402 & \{1 .-\theta\} & \{2 .++\} \\ \text { POINT } 403\{1 .-\theta\} & \{2.5-\theta\} \\ \text { POINT } 404\{0 .+\theta\} & \{2.5-\theta\}\end{array}$

LINE 401 STR $401 \quad 402010$

IINE 402 STR $402 \quad 403 \quad 0 \quad 5$

LINE 403 STR $403 \quad 404010$

LINE 404 STR $404 \quad 40105$

REGION $\begin{array}{llllll}01 & 1 & -401 & -402 & -403 & -404\end{array}$

POINT $411\{1 .+\theta\}(2 .+\theta\}$

POINT 412 (2.,-e $\{2 .+. e\}$

POINT $413(2 .-\theta\}\{2.5-\theta\}$

POINT $414\{1 .+\theta\}\{2.5-\theta\}$

LINB 411 STR 4114120010

LINE 412 STR $412 \quad 413 \quad 0 \quad 5$

IINE 113 STR $413 \quad 414$ a 10

LINE 414 STR $414 \quad 41105$

REGION $411 \quad 1 \quad-411-412 \quad-413 \quad-414$

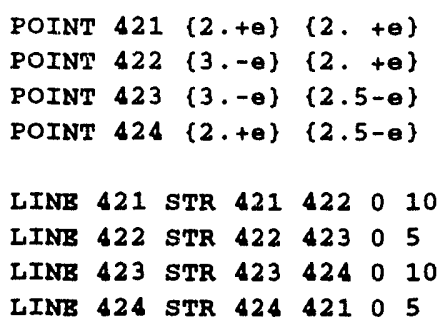

BXIT 
GEN3D file for bricks:

translate 3.6

exit

FASTQ file for wall:

title

wall

point 15.50 .

point 25.85355 .35355

point 4.55 .

point $3.85355 \quad 5.35355$

line 1 str 1205

line 2 str 233070

line 3 str 3405

line atr 41070

region $\begin{array}{llllll}1 & 3 & -1 & -2 & -3 & -4\end{array}$

exit

GEN3D file for wall:

translate 91.5

offset $0.05 \quad 0.05 \quad .5$

exit

FASTQ file for rod:

title

brick wall example

\$ STBEL ROD

POINT 5001.250

POINT 5011.750.

POINT $502 \quad 1.75 \quad-.5$

POINT $503 \quad 1.25-.5$

LINE 501 STR 50050105

LINE 502 STR 50150205

LINB 503 STR $502 \quad 503 \quad 0 \quad 5$

LINB 504 STR 50350005

RBGION $\begin{array}{llllll}500 & 2 & -501 & -502 & -503 & -504\end{array}$

$\operatorname{ExIT}$

GEN3D file for rod:

translate 305

offset 0.0 .3

exit 
GREPOS file for bricks, wall, and rod:

bricks.g

wal1.g

no

exit

$y e s$

rod.g

no

exit

no

brick_wall.g

PRONTO3D input file:

title

MULTI-BOBY CONTACT test problem

TERMINATION TIME $=.0005$

PLOT TIMB $=.00001$

OUTPUT TIME $=.00001$

MATBRIAL， 1， BLASTIC，.00074

YOUNGS MODULUS, 30B6

POISONS RATIO, . .3333

END

MATBRIAL, 2, BLASTIC, .0074

YOUNGS MODULUS, $30 \mathrm{~B} 6$

POISONS RATIO, .3333

END

MATBRIAL, 3， BLASTIC plastic, .0005

YOUNGS MODULUS, $16 \mathrm{E} 6$

POISONS RATIO, . 3333

hardening modulus 1000 .

beta $=1$

yield stress $=10000$.

END

INITIAL VBLOCITY MATERIAL $20 ., 2500 ., 0$.

PLOT BLEMENT =

PLOT NODAL DISPLACBMBNT, VBLOCITY, ACCELBRATION

CONTACT MATBRIAL 1

CONTACT MATERIAL 2

CONTACT MATERIAL 3

EXIT 


\section{A2.3.6 Forging of a Copper Billet}

FASTQ file:

\section{TITLE}

RIVBT PROBLEM FOR CONTACT SURFACE CHECK

POINT 10.00 .0

POINT 22.00 .0

POINT $3 \quad 2.20 .0$

POINT $40.0 \quad 0.2$

POINT 52 2.0 0.2

POINT $\begin{array}{lll}6 & 2.2 & 0.2\end{array}$

POINT 72.02 .95

POINT $8 \quad 2.2 \quad 3.0$

POINT $9 \quad 4.0 \quad 3.0$

POINT $10 \quad 2.0 \quad 3.2$

POINT $112.25 \quad 3.2$

POINT 124.03 .2

POINT $130.0 \quad 0.2$

POINT $14 \quad 1.75 \quad 0.2$

POINT $15 \quad 1.75 \quad 6.2$

POINT $16 \quad 0.0 \quad 6.2$

POINT 170.06 .2

POINT $18 \quad 4.0 \quad 6.2$

POINT $19 \quad 4.0 \quad 7.2$

POINT $20 \quad 0.0 \quad 7.2$

POINT $212.25 \quad 3.0$

POINT $22 \quad 2.202 .95$

POINT $23 \quad 2.25 \quad 2.95$

LINE 1 STR 1301

IINE 2 STR $322 \quad 0 \quad 1$

LINE 3 STR 219901

LINE 4 STR 45001

LINE 5 STR $5 \begin{array}{llll}7 & 0 & 1\end{array}$

LINE 6 STR $11 \quad 12 \quad 0 \quad 1$

LINE 7 STR 14001

LINE $8 \begin{array}{llllll}5 & 5 & 3 & 0 & 1\end{array}$

LINE 9 STR $810 \quad 0 \quad 1$

IINE 10 STR $912 \quad 1201$

LINE 13 STR $13 \quad 14 \quad 0 \quad 20$

$\begin{array}{lllllll}\text { LINE } & 14 & \text { STR } & 14 & 15 & 0 & 80\end{array}$

LINB 15 STR $16 \quad 1500$

LINB 16 STR $13 \quad 16 \quad 0 \quad 80$

$\begin{array}{lllllll}\text { LINE } & 17 & \text { STR } & 17 & 18 & 0 & 1\end{array}$

LINB $18 \quad \mathrm{STR} \quad 18 \quad 19 \quad 0 \quad 1$

LINB 19 STR $20 \quad 19001$

IINE 20 STR $17 \quad 20001$

IINE 21 CIRC $21 \quad 22 \quad 23 \quad 45$

LINE 22 CIRC $11 \quad 7 \quad 23 \quad 45$

$\begin{array}{lllllll}\text { LINE } 23 & \text { STR } 7 & 22 & 0 & 1\end{array}$

LINE 24 STR 2111001

RBGION $1 \begin{array}{lllll}1 & -1 & -8 & -4 & -7\end{array}$

$\begin{array}{lllllll}\text { REGION } & 2 & 1 & -2 & -23 & -5 & -8\end{array}$

$\begin{array}{lllllll}\text { REGION } 3 & 1 & -3 & -10 & -6 & -24\end{array}$

$\begin{array}{lllllll}\text { REGION } & 4 & 2 & -13 & -14 & -15 & -16\end{array}$

REGION $5 \begin{array}{llllll}5 & 3 & -17 & -18 & -19 & -20\end{array}$

REGION $6 \begin{array}{llllll}6 & 1 & -21 & -24 & -22 & -23\end{array}$

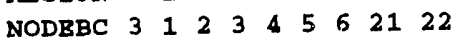

$\begin{array}{lllllll}\text { NODBBC } & 1 & 16 & 17 & 18 & 19 & 20\end{array}$

$\begin{array}{llllll}\text { NODEBC } & 2 & 17 & 18 & 19 & 20\end{array}$

SIDEBC 10045622

SIDEBC $200 \quad 13 \quad 14 \quad 15$

SIDEBC 30017

SIDEBC $400 \quad 14 \quad 15$

EXIT 
SANTOS2D input file:

TITLE

RIVET PROBLEM - CHECK FOR CONTACT SURPACBS

AXISYMMETRIC

STRP CONTROL

5001 .

BND

OUTPUT TIME

101.

END

PLOT TIME

51.

BND

TIME STEP SCALE $=0.75$

MINIMUM DAMPING FACTOR $=.8$

RESIDUAL TOLERANCE, 0.5

MAXIMUM TOLERANCE, 100 .

MAXIMUM ITERATIONS, 2000

INTERMEDIATB PRINT, 10

PLOT NODAL $=$ DISPLACBMRNT

PLOT STATE $=$ EQPS

PLOT BLEMENT = VONMISES, PRESSURR, STRBSS, STRAIN

EUNCTION, 1

$0.0,0.0$

$1.0,1.0$

END

MATERIAL， 1， BLASTIC, 1

YOUNGS MODULUS $=30 . \mathrm{E} 6$

POISSONS RATIO $=0.3$

BND

MATERIAL, 2, EP POWER HARD, 1.

YOUNGS MODULUS $=17 \cdot 0 \mathrm{~B}+6$

POISSONS RATIO $=0.35$

HARDENING CONSTANT $=50.0 \mathrm{~B}+3$

HARDBNING EXPONENT $=.31$

YIELD STRESS $=15.5 \mathrm{~B}+3$

LUDER STRAIN $=0.0$

END

MATERIAL, 3, BLASTIC, 1.

YOUNGS MODULUS $=30 . \mathrm{E}+6$,

POISSONS RATIO $=1.3$

BND

CONTACT SUREACE, 100, 200, 0., 1.B-10

CONTACT SURFACE, 300,400, 0., 1.B-10

NO DISPLACEMBNT, $X, 3$

NO DISPLACEMENT, $Y, 3$

NO DISPLACEMBNT, $\mathrm{X}, 1$

PRESCRIBED DISPLACEMENT, $Y, 2,1,-2.5$

BXIT 


\section{DISTRIBUTION:}

1 Dr. R. T. Allen

Pacifica Technology

P.O. Box 148

Del Mar, CA 92014

3 Anatech International Corp.

5435 Oberlin Drive

San Diego, CA 92121

Attn: Dr. R. A. Dameron

Dr. R. S. Dunham

Dr. Joe Rashid

Prof. S. Atluri

Center for the Advancement of Computational Mechanics

School of Civil Engineering

Georgia Institute of Technology

Atlanta, GA 30332

Dr. Ali S. Argon

Dept. of Mechanical Engineering

Massachusetts Institute of

Technology

Cambridge, MA 02139

Dr. William E. Bachrach

Areojet Research Propulsion Inst.

P. O. Box 13502

Sacramento, CA 95853-4502

Mr. Ken Bannister

USA Ballistic Research Lab

SLCBR-IB-M

Aberdeen Proving Grounds, MD

21005-5066

3 Battelle

505 King Avenue

Columbus, OH 43201-2693

Attn: Mr. R. Douglas Everhart

Dr. Michael L. Fisher

Mr. Charles R. Hargreaves
Prof. T. Belytschko

Department of Civil Engineering

Northwestern University

Evanston, IL 60201

Mr. Willy Benz

University of Arizona

Steward Observatory

Tucson, AZ 85321

Mr. Naury K. Birnbaum

Century Dynamics Incorporated

7700 Edgewater Dr., Suite 626

Oakland CA 94621

Mr. Akif O. Bolukbasi

McDonnell Douglas Helicopter

Company

5000 East McDowell Road

Mesa, AZ 85205-9797

Dr. Kenneth W. Brown

Computer Aided Engineering

Associates, Inc.

398 Old Sherman Hill Rd.

Woodbury, CT 06798

Mr. Malcolm Burgess

AEA Transport Technology

205/B71 Winfrith Technology Centre

Dorchester, Dorset DT2 8DH

United Kingdom

Mr. Mark Campbell

PASTDCO

1248 Princeton N.E.

Albuquerque, NM 87106

Dr. Tom Canfield

Argonne National Laboratories

9700 S. Cass Ave CTD/221

Argonne, IL 60439-4844 
Mr. Ted Carney

Advanced Sciences Inc.

6739 Academy Road N.E.

Albuquerque, NM 87109

Mr. Tien S. Chou

EG\&G Mound

P.O. Box 3000

Miamisburg, OH 45343

Mr. Chuck Charman

GA Technologies

P.O. Box 81608

San Diego, CA 92138

Mr. Ken K. Chipley

Martin Marietta Energy Systems

P.O. Box 2009

Oak Ridge, TN 37831-8053

Mr. Ken P. Chong

Dept. of Civil Engineering

University of Wyoming

Laramie, WY 82071

Dr. S. C. (Tony) Chou

U.S. Army Materials Technology Lab

SLCMT-BM

Watertown, MA 02172-0001

Mr. Dwight Clark

Mail Stop 281

Morton Thiokol Corp.

P. O. Box 524

Brigham City, UT 84302

Mr. Gregory Clifford

Cray Research Park

655E Lone Oak Drive

Eagan, MN 55121

Mr. Gerald Collingwood

Morton Thiokol, Inc.

Huntsville, AL 35807-7501
Mr. David L. Conover

Swanson Analysis Systems, Inc.

P. O. Box 65

Houston, PA 15342-0065

Prof. Steven M. Cramer

University of Wisconsin

2266 Engineering Building

1415 Johnson Drive

Madison, WI 53706

Mr. Steven Crouch

GeoLogic Research, Inc.

1313 Fifth St. SE, Suite 226

Minneapolis, MN 55414

Dr. Ian Cullis

XTZ Division

Royal Armament R\&D

Establishment

Fort Halstead

Sevenoaks, Kent

United Kingdom

Mr. Peter Cundall

ITASCA Consulting Group, Inc.

1313 Fifth Street, S.E.

Minneapolis, MN 55414

Mr. Richard E. Danell

Research Officer

Central Research Laboratories

BHP Research \& New Technology

P.O. Box 188

Wallsend NSW 2287

Australia

Mr. William A. Danne

Strategic Systems Division, MS\#50

Teledyne Brown Engineering

P.O. Box 07007

Huntsville, AL 35807-7007 
Dr. C. S. Desai

Dept. of Civil Eng. \& Eng. Mech.

The University of Arizona

Tucson, AZ 85721

Mr. Ramji Digumarthi

Org. 8111, Bldg. 157

Lockheed MSD

P.O. Box 3504

Sunnyvale, CA 94088-3504

Prof. Robert Dodds, Jr.

Department of Civil Engineering

3140 Newmark Laboratory, MC-250

University of Illinois at Urbana

Urbana, IL 61801-2397

Dr. Arlo Fossum

RE/SPEC Inc.

Box 725

Rapid City, SD 57709

Dr. Russel Garnsworthy

CRA Advanced Tech Development

G.P.O. Box 384D

Melbourne 3001, Australia

Prof. Lorna Gibson

Department of Civil Engineering

Room 1-232

Massachusetts Institute of Technology

Cambridge, MA 02139

2 Goodyear Technical Center

P.O. Box 3531

Akron, $\mathrm{OH}$ 44309-3531

Attn: Mr. Loren K. Miller

Mr. David Wismer, D/410F

Dr. Gerry Goudreau

Methods Development Group

Mechanical Engineering Department

Lawrence Livermore National Lab

Livermore, CA 94550
Prof. O. Hayden Griffin, Jr.

Dept. of Eng. Science \& Mechanics

Virginia Polytechnic Institute and

State University

Blacksburg, VA 24061-0219

2 Grumman Corporate Technology

Bethpage, NY 11714-3580

Attn: Dr. John M. Papazian

Dr. Allan B. Pifko

Mr. H. L. Hassenpflug

B\&W Fuel Company

P.O. Box 10935

3315 Old Forest Rd

Lynchburg, VA 24501

5 Hibbitt, Karlsson \& Sorrensen, Inc.

100 Medway St.

Providence, RI 02906

Attn: Dr. David Hibbitt

Dr. Joop Nagtegaal

Dr. D. P. Flanagan

Dr. L. M. Taylor

Dr. W. C. Mills-Curran

Mr. Richard Hilson

M/S 4G09

GTE Government Systems

Corporation

P.O. Box 7188

Mountain View, CA 94039

Mr. Douglas Holzhauer

Rome Air Development Center

Griffiss AFB, NY 13441

Dr. William Hufferd

United Technologies

Chemical Systems Division

P.O. Box 50015

San Jose, CA 95150-0015 
Prof. T. J. R. Hughes

Dept. of Mechanical Engineering

Stanford University

Palo Alto, CA 94306

Mr. James P. Johnson

Rm L120, CPC Analysis Dept.

General Motors Corp. Engineering

Center

30003 Van Dyke Avenue

Warren, MI 48090-9060

Mr. Jerome B. Johnson

USACRREL

Building 4070

Ft. Wainwright, AK 99703

Mr. Ken Johnson

Theoretical and Applied Mechanics Group

Battelle Pacific Northwest

Laboratories

P.O. Box 999

Richland, WA 99352

Dr. Gordon R. Johnson

Honeywell, Inc.

5901 S. County Rd. 18

Edina, MN 55436

Mr. James W. Jones

Swanson Service Corporation

18700 Beach Blvd.

Suite 200-210

Huntington Beach, CA 92648

Mr. Sheldon Jones

Kaman Sciences

P.O. Box 7463

Colorado Springs, CO 80933-7463

Mr. G. A. Kaepp

Ford Motor Company

P.O. Box 2053, Room 2019

Dearborn, MI 48124
Dr. David W. Keck

CONVEX Computer Corporation

P.O. Box 833851 M.S. MAR

Richardson, TX 75083-3851

Mr. Gary Ketner

Applied Mechanics and Structures

Battelle Pacific Northwest

Laboratories

P.O. Box 999

Richland, WA 99352

Dr. Sam Key

RE/SPEC Inc.

4775 Indian School NE, Suite 300

Albuquerque, NM 87110-3827

Prof. Raymond D. Krieg

Engineering Science and Mechanics

301 Perkins Hall

University of Tennessee

Knoxville, TN 37996-2030

Mr. Don D. Kunard

Analytical Systems Engineering

Corp.

1725 Jefferson Davis Hwy, Suite 212

Arlington, VA 22202

Mr. Brett Lewis

APTEK

1257 Lake Plaza Drive

Colorado Springs, CO 80906-3578

Norman A. Lindsey

MCAE Technical Marketing

CONVEX Computer Corporation

P. O. Box 833851 M.S. MAR

Richardson, TX 75083-3851

Mr. Trent R. Logan

Rockwell International Corp.

P.O. Box 92098

Los Angeles, CA 90009 
34 Los Alamos National Laboratory Los Alamos, NM 87545

Attn:

J. Hopson, T3, MS B216

R. Hill, P15, MS D44

J. P. Hill, WX-11, MS C931

D. J. Sandstorm, MST-DO, MSG756

K. A. Meyer, X-3 MS F663

W. A. Cook, N-6, MS K557

P. T. Maulden, N-6, MS K557

J. J. Ruminer, WX-11, MS C931

S. P. Girrens, MEE-13, MS J576

J. L. Fales, MEE-13, MS J575

J. D. Allen, MEE-4, MS G787

D. A. Rabern, MEE-4, MS G787

M. W. Burkett, MEE-4, MS G787

J. H. Fu, MEE-4, MS G787

P. R. Romero, MEE-4, MS G787

P. S. Follansbee, MST-DO, MS G756

D. Mandell, X-3, MS F663

R. F. Davidson, N-6, MS K557

J. N. Johnson, N-6, MS K557

J. K. Dienes, N-6, MS K557

S. Marsh, N-6, MS K557

L. H. Sullivan, N-6, MS K557

D. L. Jaeger, WX-11, MS K557

C. A. Anderson, MEE-13, MS J576

J. G. Bennett, MEE-13, MS J576

T. A. Butler, MEE-13, MS J576

D. C. Nelson, MEE-4, MS G787

R. B. Parker, MEE-4, MS G787

M. W. Lewis, MEE-4, MS G787

E. S. Idar, MEE-4, MS G787

B. M. Wheat, MEE-4, MS G787

F. Guerra, WX-11, MS C931

C. Wingate, MS F645

B. Stellingwerf, MS F645

Dr. Jack Maison

Engineering Cybernetics, Inc.

1856 Lockhill Selma Rd, Suite 105

San Antonio, TX 78213
Mr. Joseph Marti

Hamilton Standard Division of

United Technologies

M/S 1-3-BC52

One Hamilton Road

Windsor Locks, CT 06096-1010

Mr. Darin McKinnis

NASA Pyrotechnics Group, MS EP5

LBJ Space Center

Houston, TX 77058

Mr. Craig Miller

Unit 973

Neutron Devices Department

General Electric Company

P.O. Box 2908

Largo, FL 34294-2908

2 Lockheed Missiles and Space Co.

P. O. Box 3504

Sunnyvale, CA 94088-3504

Attn:

Mr. J. J. Murphy, 59-22 B/580

Mr. Brian M. Cuthbert, 81-12 B/157

Prof. V. D. Murty

School of Engineering

University of Portland

5000 N. Willamette Blvd.

Portland, OR 97203

2 Naval Surface Warfare Center

10901 New Hampsure Ave.

Silver Spring, MD 20903-5000

Attn:

Mr. Hans Mair, Code R14

Mr. Andrew Wardlaw Jr. Code R44

2 Naval Research Lab

Materials Science \& Technology

Building 28, Code 6386

4555 Overlook Avenue SW

Washington, DC 20375-5000

Attn: Mr. Luther D. Flippen

Dr. Carl Dyka 
Dr. R. E. Nickell

c/o Anatech International Corp.

5435 Oberlin Drive

San Diego, CA 92121

Mr. Dean Norman

Waterways Experiment Station

P.O. Box 631

Vicksburg, MS 39180

2 Office of Naval Research

Structural Mechanics Div. (Code 434)

800 N. Quincy Street

Arlington, VA 22217

Attn: Dr. Rembert Jones

Dr. Alan S. Kushner

Mr. Shane R. Page

Albuquerque Valve \& Fitting

Company

2451 Alamo S.E.

Albuquerque, NM 87106

Dr. Robert Pardue

Martin Marietta, MS 2

Y-12 Plant, Bldg. 9998

Oak Ridge, TN 37831

Dr. T. Kim Parnell

Failure Analysis Associates, Inc.

P.O. Box 3015

Menlo Park, CA 94025

Dr. Philip A. Pfund

Babcock \& Wilcox

P.O. Box 271

Barberton, $\mathrm{OH} 44203$

Mr. Mitchell R. Phillabaum

Monsanto Research Corp.

MRC-MOUND

Miamisburg, OH 45342
4 Phillips Laboratory (AFSC)

Kirtland AFB, NM 87117-6008

Attn:

Firooz Allahdadi, PL/WSSD

David Amdahl, PL/WSSD

David Medina, PL/WSSD

David H. Hilland, PL/WSSH

3 POD Associates, Inc.

2309 Renard Pl, Suite 201

Albuquerque, NM 87106

Attn: Mr. Dale R. Atkinson

Mr. Steven F. Rieco

Dr. Alan J. Watts

2 Pratt \& Whitney Aircraft 400 Main St.

East Hartford, CT 06108

Attn: John Cowles, MS 118-38

Mick Bruskotter, MS 114-38

Dr. Harold E. Read

S-Cubed

P.O. Box 1620

La Jolla, CA 92038-1620

Dr. Douglas Reeder

Hardening Technology Dept.

General Research Corp.

P.O. Box 6770

Santa Barbara, CA 93160-6770

Prof. J. A. Reuscher

Texas A \& M

Dept. of Nuclear Engineering

College Station, Texas 77843

2 Reynolds Metals Company

1941 Reymet Road

Richmond, VA 23237

Attn: Mr. Stephen P. Sunday

Mr. Armand Beaudoin 
Mr. J. S. (Gus) Rice

Caterpillar Inc. Technical Center

Division 927

P.O. Box 1875

Peoria, IL 61656-1875

Mr. Samit Roy

Dept. of Engineering Mechanics

Southwest Research Institute

P.O. Drawer 28510

San Antonio, TX 78284

R. G. Sauvé

Applied Mechanics Section

Mechanical Research Department

Ontario Hydro

700 University Avenue

C26

Toronto, Ontario M5G 1X6

Canada

Mr. Donald W. Sandidge

AMSMI-RLA

U.S. Army Missile Command

Redstone Arsenal, AZ 35898-5247

Mr. Steven Sauer

Ktech Corporation

901 Pennsylvania Ave NE

Albuquerque, NM 87110

Mr. Martin Schmidt, M/S 4G09

WL/MNSA

Eglin AFB, FL 32542-5434

Mr. Luka Serdar, Jr.

Kaman Sciences Corporation

AviDyne Office

83 Second Ave

Burlington, MA 01803-4479

Mr. Harvey Singer

Science Applications International

P.O. Box 1303

McLean, VA 22102-1303
Mr. Mark E. Smith

Arvin Calspan Corp.

AEDC Division, M/S 440

Arnold AFB, TN 37389-9998

Mr. Ray Stoudt

Lawrence Livermore National Lab

P.O. Box 808, L200

Livermore, CA 94550

Prof. D. V. Swenson

Mechanical Engineering Department

Kansas State University

Manhattan, KS 66506

Mr. David W. Sykora

U.S. Army Corps of Engineers

Waterways Experimental Station

P.O. Box 631

Vicksburg, MS 39180

Mr. Sing C. Tang

Rm 3039 Scientific Lab

P. O. Box 2053

Dearborn, MI 48121-2053

2 Spokane Research Center

U.S. Bureau of Mines

315 Montgomery Avenue

Spokane, WA 99207-2291

Attn: Mr. J. Donald Dixon

Dr. Hamid Maleki

2 TRW Ballistic Missiles Division

Bldg 527, Rm 709

P.O. Box 1310

San Bernadino, CA 92402

Attn: Dr. Mike Katona

Mr. Richard Lung 


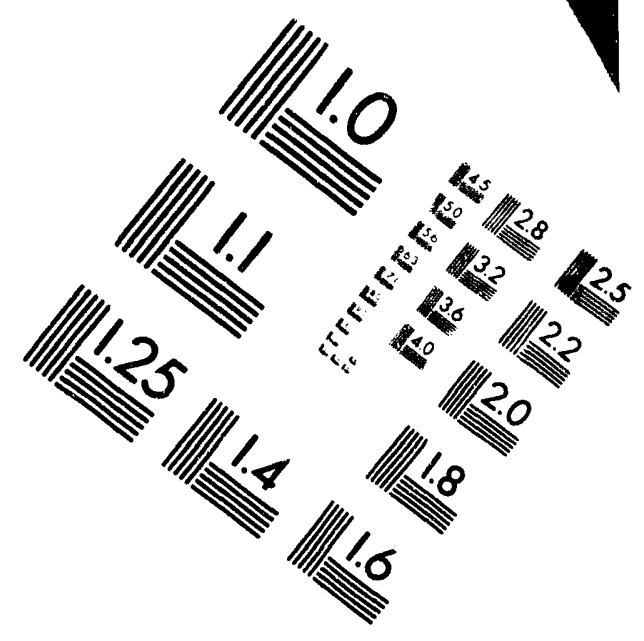

\section{Centimeter}

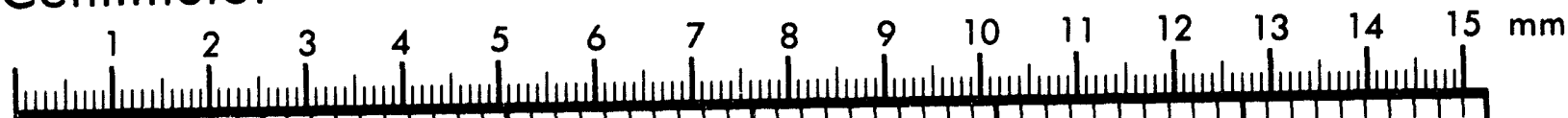

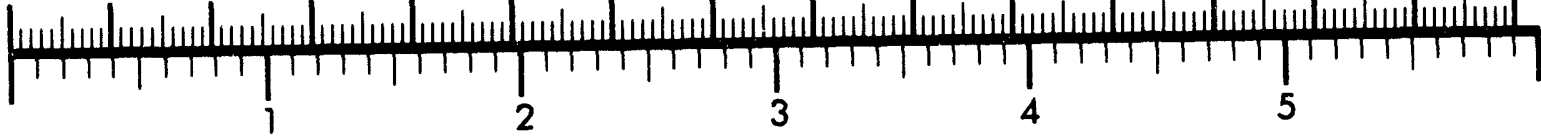
Inches
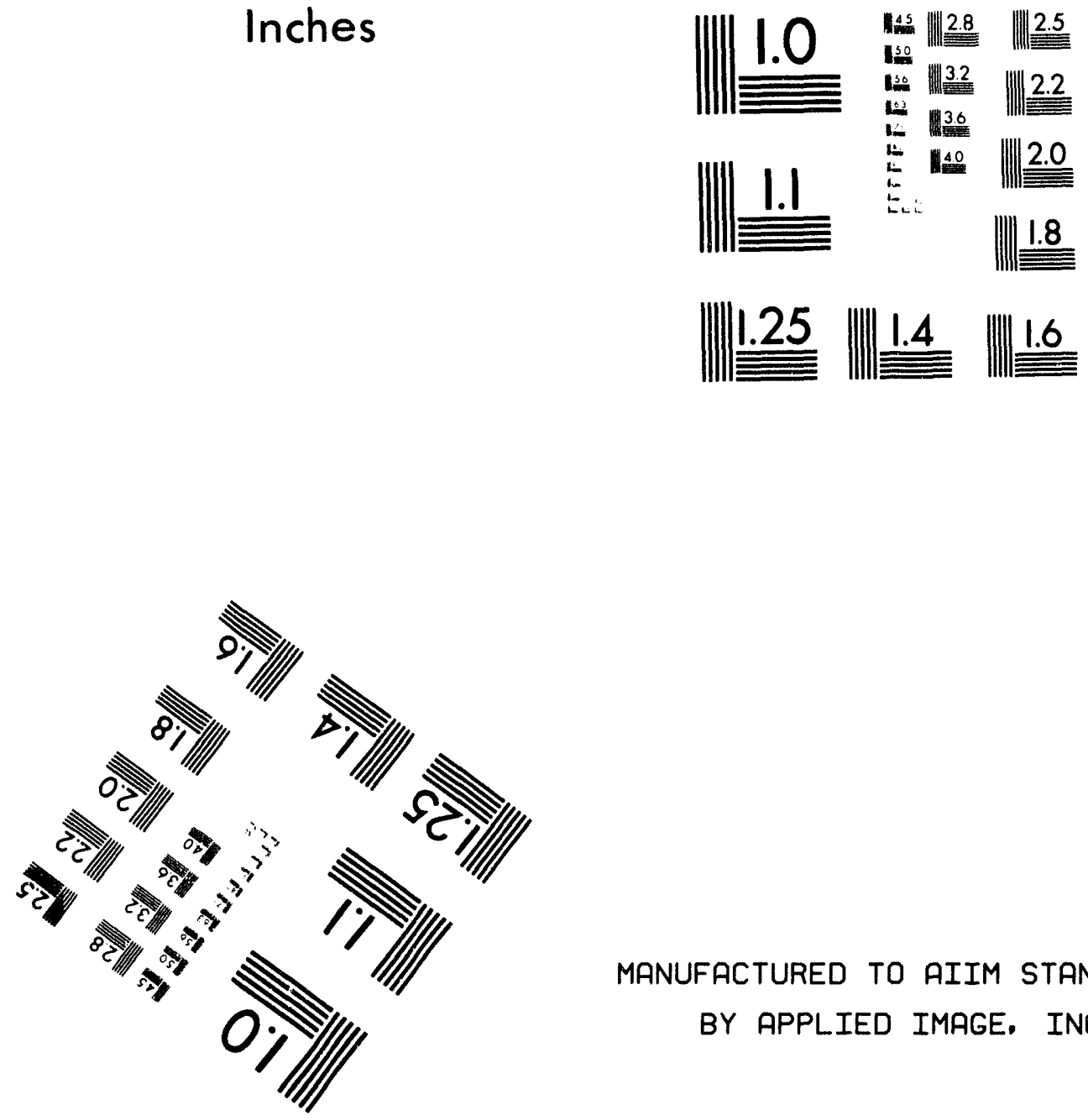

MANUFACTURED TO AIIM STANDARDS

BY APPLIED IMAGE. INC.

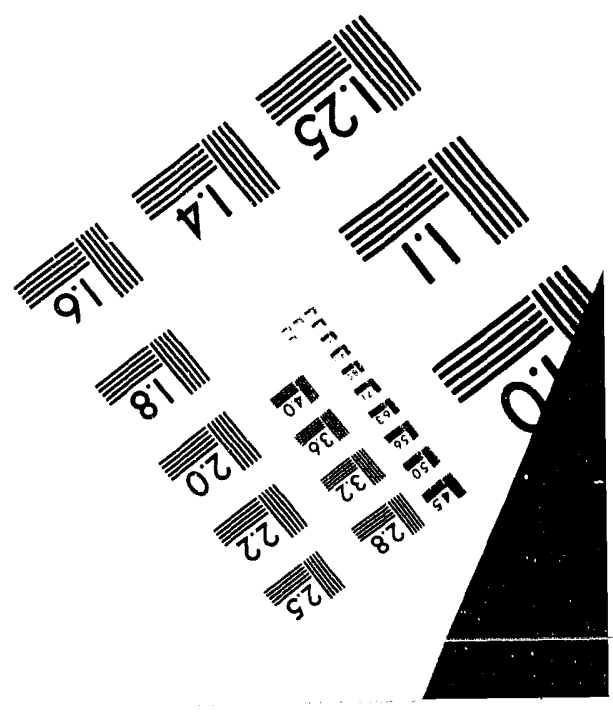



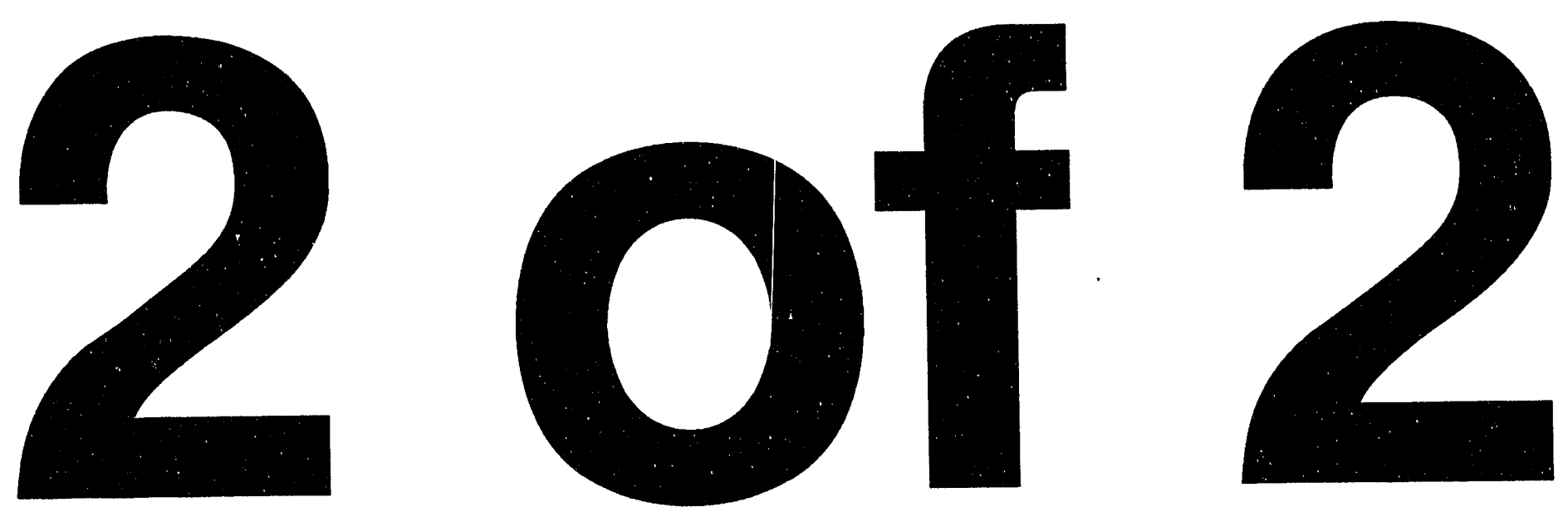
34 Los Alamos National Laboratory

Los Alamos, NM 87545

Attn:

J. Hopson, T3, MS B216

R. Hill, P15, MS D44

J. P. Hill, WX-11, MS C931

D. J.Sandstorm, MST-DO, MSG756

K. A. Meyer, X-3 MS F663

W. A. Cook, N-6, MS K557

P. T. Maulden, N-6, MS K557

J. J. Ruminer, WX-11, MS C931

S. P. Girrens, MEE-13, MS J576

J. L. Fales, MEE-13, MS J575

J. D. Allen, MEE-4, MS G787

D. A. Rabern, MEE-4, MS G787

M. W. Burkett, MEE-4, MS G787

J. H. Fu, MEE-4, MS G787

P. R. Romero, MEE-4, MS G787

P. S. Follansbee, MST-DO, MS G756

D. Mandell, X-3, MS F663

R. F. Davidson, N-6, MS K557

J. N. Johnson, N-6, MS K557

J. K. Dienes, N-6, MS K557

S. Marsh, N-6, MS K557

L. H. Sullivan, N-6, MS K557

D. L. Jaeger, WX-11, MS K557

C. A. Anderson, MEE-13, MS J576

J. G. Bennett, MEE-13, MS J576

T. A. Butler, MEE-13, MS J576

D. C. Nelson, MEE-4, MS G787

R. B. Parker, MEE-4, MS G787

M. W. Lewis, MEE-4, MS G787

E. S. Idar, MEE-4, MS G787

B. M. Wheat, MEE-4, MS G787

F. Guerra, WX-11, MS C931

C. Wingate, MS F645

B. Stellingwerf, MS F645

Dr. Jack Maison

Engineering Cybernetics, Inc.

1856 Lockhill Selma Rd, Suite 105

San Antonio, TX 78213
Mr. Joseph Marti

Hamilton Standard Division of

United Technologies

M/S 1-3-BC52

One Hamilton Road

Windsor Locks, CT 06096-1010

Mr. Darin McKinnis

NASA Pyrotechnics Group, MS EP5

LBJ Space Center

Houston, TX 77058

Mr. Craig Miller

Unit 973

Neutron Devices Department

General Electric Company

P.O. Box 2908

Largo, FL 34294-2908

2 Lockheed Missiles and Space Co.

P. O. Box 3504

Sunnyvale, CA 94088-3504

Attn:

Mr. J. J. Murphy, 59-22 B/580

Mr. Brian M. Cuthbert, 81-12 B/157

Prof. V. D. Murty

School of Engineering

University of Portland

$5000 \mathrm{~N}$. Willamette Blvd.

Portland, OR 97203

2 Naval Surface Warfare Center

10901 New Hampsure Ave.

Silver Spring, MD 20903-5000

Attn:

Mr. Hans Mair, Code R14

Mr. Andrew Wardlaw Jr. Code R44

2 Naval Research Lab

Materials Science \& Technology

Building 28, Code 6386

4555 Overlook Avenue SW

Washington, DC 20375-5000

Attn: Mr. Luther D. Flippen

Dr. Carl Dyka 
Dr. R. E. Nickell

c/o Anatech International Corp.

5435 Oberlin Drive

San Diego, CA 92121

Mr. Dean Norman

Waterways Experiment Station

P.O. Box 631

Vicksburg, MS 39180

2 Office of Naval Research

Structural Mechanics Div. (Code 434)

800 N. Quincy Street

Arlington, VA 22217

Attn: Dr. Rembert Jones

Dr. Alan S. Kushner

Mr. Shane R. Page

Albuquerque Valve \& Fitting

Company

2451 Alamo S.E.

Albuquerque, NM 87106

Dr. Robert Pardue

Martin Marietta, MS 2

Y-12 Plant, Bldg. 9998

Oak Ridge, TN 37831

Dr. T. Kim Parnell

Failure Analysis Associates, Inc.

P.O. Box 3015

Menlo Park, CA 94025

Dr. Philip A. Pfund

Babcock \& Wilcox

P.O. Box 271

Barberton, OH 44203

Mr. Mitchell R. Phillabaum

Monsanto Research Corp.

MRC-MOUND

Miamisburg, OH 45342
4 Phillips Laboratory (AFSC)

Kirtland AFB, NM 87117-6008

Attn:

Firooz Allahdadi, PL/WSSD

David Amdahl, PL/WSSD

David Medina, PL/WSSD

David H. Hilland, PL/WSSH

3 POD Associates, Inc.

2309 Renard Pl, Suite 201

Albuquerque, NM 87106

Attn: Mr. Dale R. Atkinson

Mr. Steven F. Rieco

Dr. Alan J. Watts

2 Pratt \& Whitney Aircraft 400 Main St.

East Hartford, CT 06108

Attn: John Cowles, MS 118-38

Mick Bruskotter, MS 114-38

Dr. Harold E. Read

S-Cubed

P.O. Box 1620

La Jolla, CA 92038-1620

Dr. Douglas Reeder

Hardening Technology Dept.

General Research Corp.

P.O. Box 6770

Santa Barbara, CA 93160-6770

Prof. J. A. Reuscher

Texas A \& M

Dept. of Nuclear Engineering

College Station, Texas 77843

2 Reynolds Metals Company

1941 Reymet Road

Richmond, VA 23237

Attn: Mr. Stephen P. Sunday

Mr. Armand Beaudoin 
Mr. J. S. (Gus) Rice

Caterpillar Inc. Technical Center

Division 927

P.O. Box 1875

Peoria, IL 61656-1875

Mr. Samit Roy

Dept. of Engineering Mechanics

Southwest Research Institute

P.O. Drawer 28510

San Antonio, TX 78284

R. G. Sauvé

Applied Mechanics Section

Mechanical Research Department

Ontario Hydro

700 University Avenue

C26

Toronto, Ontario M5G 1X6

Canada

Mr. Donald W. Sandidge

AMSMI-RLA

U.S. Army Missile Command

Redstone Arsenal, AZ 35898-5247

Mr. Steven Sauer

Ktech Corporation

901 Pennsylvania Ave NE

Albuquerque, NM 87110

Mr. Martin Schmidt, M/S 4G09

WL/MNSA

Eglin AFB, FL 32542-5434

Mr. Luka Serdar, Jr.

Kaman Sciences Corporation

AviDyne Office

83 Second Ave

Burlington, MA 01803-4479

Mr. Harvey Singer

Science Applications International

P.O. Box 1303

McLean, VA 22102-1303
Mr. Mark E. Smith

Arvin Calspan Corp.

AEDC Division, M/S 440

Arnold AFB, TN 37389-9998

Mr. Ray Stoudt

Lawrence Livermore National Lab

P.O. Box 808, L200

Livermore, CA 94550

Prof. D. V. Swenson

Mechanical Engineering Department

Kansas State University

Manhattan, KS 66506

Mr. David W. Sykora

U.S. Army Corps of Engineers

Waterways Experimental Station

P.O. Box 631

Vicksburg, MS 39180

Mr. Sing C. Tang

Rm 3039 Scientific Lab

P. O. Box 2053

Dearborn, MI 48121-2053

2 Spokane Research Center

U.S. Bureau of Mines

315 Montgomery Avenue

Spokane, WA 99207-2291

Attn: Mr. J. Donald Dixon

Dr. Hamid Maleki

2 TRW Ballistic Missiles Division

Bldg 527, Rm 709

P.O. Box 1310

San Bernadino, CA 92402

Attn: Dr. Mike Katona

Mr. Richard Lung 
4 United Technologies Research Center 411 Silver Lane

East Hartford, CT 06108

Attn: Dave Edwards, MS 129-13

Robert LaBarre, MS 129-20

Tony Giamei, MS 129-22

Tom Vasko

2 Department of Applied Mechanics and Engineering Sciences

University of California San Diego

La Jolla, CA 92093

Attn: Prof. S. Nemat-Nasser Prof. Dave Benson

3 Department of Aerospace Engineering and Engineering Mechanics

The University of Texas at Austin Austin, TX 78712-1085

Attn: Prof. E. B. Becker

Prof. J. T. Oden

Prof. M. Stern

Mr. David Wade, 36E

Bettis Atomic Power Laboratory

P.O. Box 79

West Miffland, PA 15122

Dr. Krishan K. Wahi

Gram, Inc.

1709 Moon NE

Albuquerque, NM 87112

Dr. Paul T. Wang

Fabricating Technology Division

Alcoa Technical Center

Alcoa Center, PA 15069

Dr. Ted B. Wertheimer

MARC Analysis Research

Corporation

260 Sheridan Ave, Suite 309

Palo Alto, CA 94306
4 Westinghouse Electric Corporation

Bettis Atomic Power Laboratory

P.O. Box 79

West Mifflin, PA 15122-0079

Atin: Todd Hoover

Claire Knolle

Dan Kotcher

Wayne Long

Prof. Tomasz Wierzbicki

Dept. of Ocean Engineering

Massachusetts Institute of

Technology

Cambridge, MA 02139

Prof. John Wilson

Department of Geoscience

NM Institute of Mining \&

Technology

Socorro, NM 87801

Mr. Philip J. Winters

Chicago Bridge \& Iron

1501 North Division Street

Plainfield, IL 60544

Dr. John F. Wohler

Land Systems Division

General Dynamics

P.O. Box 2074

Warren, MI 48090-2074

Dr. Albert Yao

SMCRI-SEE-A

Rock Island Arsenal

Rock Island, IL 61299-5000

Mr. Jerry Zimmerlee

Manager, Engineering Analysis

Johnson Controls, Inc.

P. O. Box 8010

Plymouth, MI 48170 
Mr. J. Â. Zukas

Computational Mechanics

Consultants, Inc.

8600 La Salle Road, Suite 614

Towson, MD 21204

1200 George Allshouse

1239 Frank Dempsey

1400 Ed Barsis

1425 Johnny Biffle

101425 Stephen Attaway

1425 Mark Blanford

1425 William Bohnhoff

$50 \quad 1425 \quad$ Marilyn Smith

1431 Michael McGlaun

1431 James Peery

1434 David Martinez

1500 D. J. McCloskey

1501 Carl Peterson

1502 Paul Hommert

1511 J. S. Rottler

1512 A. C. Ratzel

1513 R. D. Skocypec

1551 W. P. Wolfe

1552

1553

1554

$\begin{array}{ll}15 & 1561\end{array}$

$10 \quad 1561$

$14 \quad 1562$

21562

$\begin{array}{ll}2 & 1562\end{array}$

1832

1912

2565

2814

5600

5941

6000

6112

6112

6113

6113
C. E. Hailey

W. L. Hermina

W. H. Rutledge

Harold Morgan and Staff

Martin Heinstein

Robert Thomas and Staff

Frank Mello

Jeff Swegle

Jeanne Ramage

William Mason

Stephen Montgomery

Randall Lober

Dennis Hayes

John Schamaun

Dan Hartley

Dale Preece

Norman Warpinski

Stephen Bauer.

Brian Ehgartner
6313 John Holland

6313 Joseph Jung

6313 John Pott

6313 Alex Treadway

6316 Mike Wernig

6500 James Rice

6514 Jim Fisk

6515 Mike Rightley

6522 Joel Miller

6642 Douglas Ammerman

57141 Technical Library

7151 Technical Publications

10 7613-2 Document Processing for DOE/OSTI

8523-2 Central Technical Files

8702 Bill Robinson

8712 Kim Mahin

8240 George Johnson

8741 Michael Chiesa

8742 Bruce Kistler

8743 Melvin Callabresi

8743 Douglas Bammann

8743 Juanita Benson

8743 Lee Bertram

8743 Mark Horstemeyer

8743 James Lathrop

8744 Arthur Ortega

8745 William Winters 

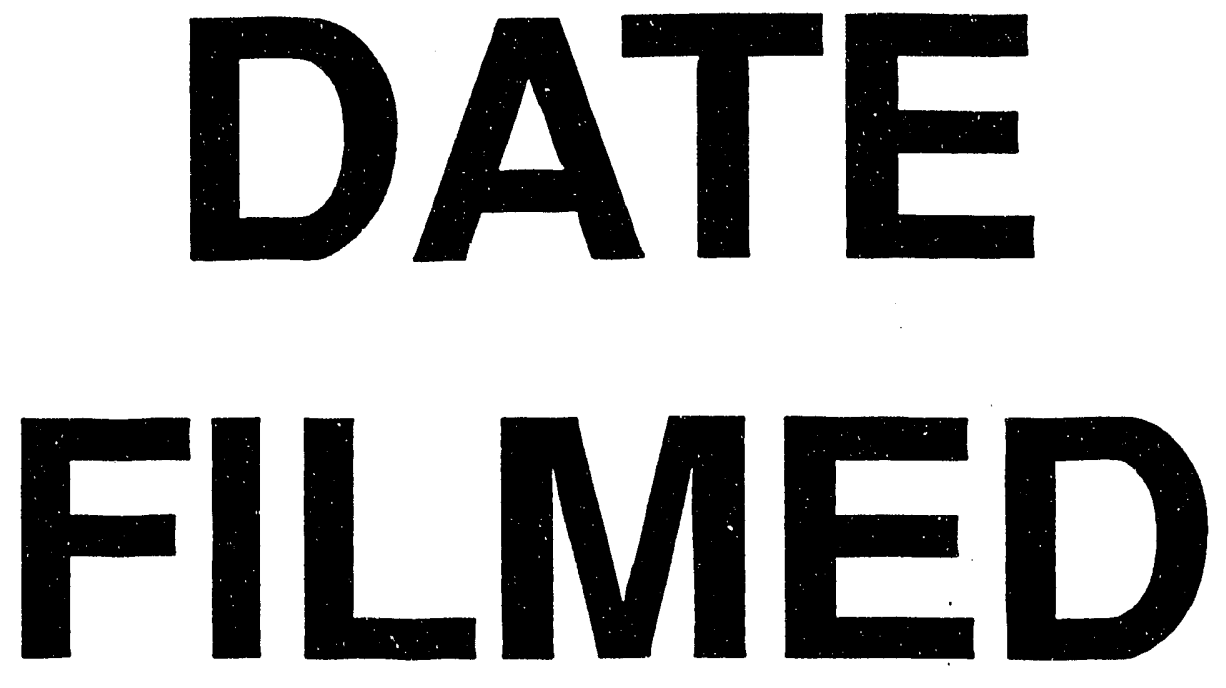

$9 / 21 / 93$
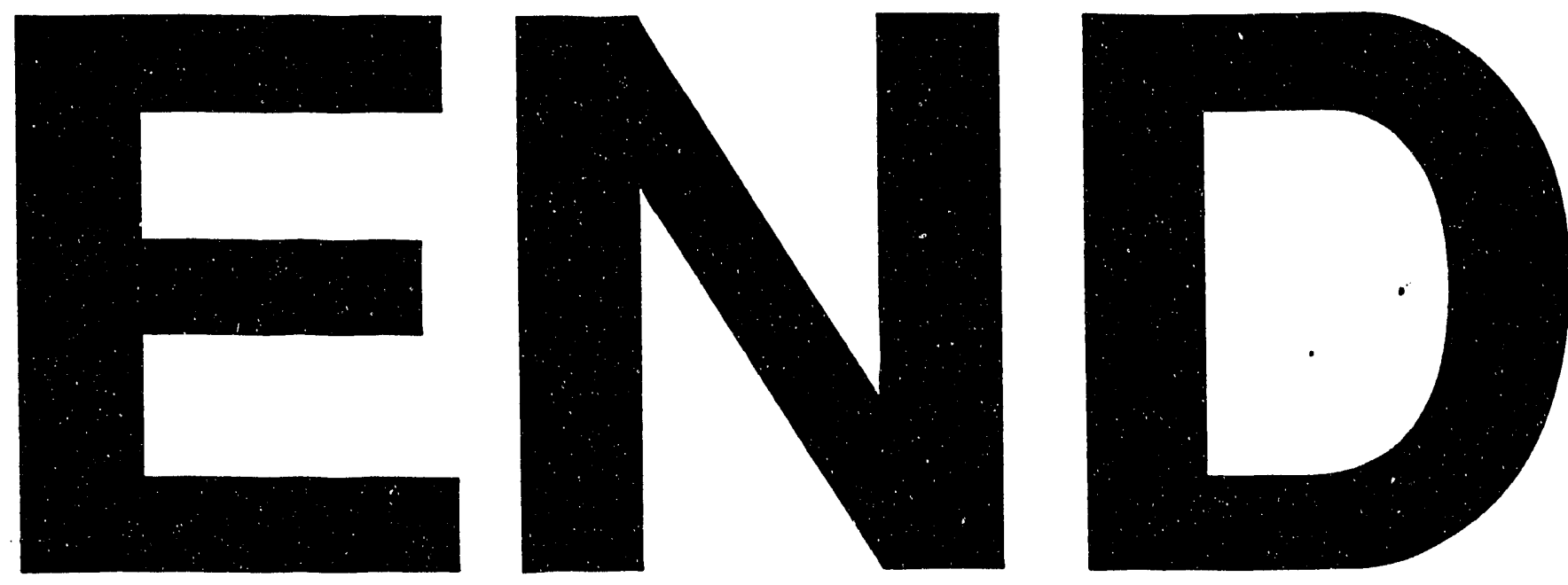
